Interfaces and Free Boundaries 17 (2015), 189-232

DOI $10.4171 / \mathrm{IFB} / 339$

\title{
Computational anisotropic Willmore flow
}

\author{
PAOLA POZZI \\ Fakultät für Mathematik, Universität Duisburg-Essen, Thea-Leymann-Straße 9, 45127 Essen, \\ Germany \\ E-mail: paola.pozzi@uni-due.de
}

[Received 9 April 2014 and in revised form 8 January 2015]

\begin{abstract}
We study a geometrically consistent formulation of anisotropic Willmore flow for parametric hypersurfaces in $\mathbb{R}^{n}$. After giving a mixed formulation that is suitable for discretization by piecewise linear finite elements, we provide a stable semi-discrete scheme. An experimentally stable fully discrete semi-implicit scheme is then discussed and several tests for the evolution of curves and surfaces are presented.
\end{abstract}

2010 Mathematics Subject Classification: Primary 35K55, 65M60, 65M12.

Keywords: Geometric evolution equation, fourth order parabolic problem, anisotropic Willmore functional, mixed method, finite elements, stability.

\section{Introduction}

Aim of this work is to provide a suitable finite element discretization of a geometrically consistent anisotropic Willmore flow for parametric hypersurfaces in $\mathbb{R}^{n}$.

First of all recall that the classical (isotropic) Willmore functional

$$
W(\mathbf{x})=\frac{1}{2} \int_{\tilde{\Gamma}} h^{2} d V
$$

is defined as the integral of the squared mean curvature $h$ of a smooth compact orientable hypersurface $\tilde{\Gamma}$ isometrically immersed in space through the map $\mathbf{x}: \tilde{\Gamma} \rightarrow \mathbb{R}^{n}$. In the past years the above functional and its related flow have been studied extensively both from an analytical and numerical point of view. Motivations for this intense study include the role of (1.1) as a prototype for a fourth order geometric problem and its applications to image processing and modeling of biological membranes (see for instance the extensive reference list provided in [15]).

On the contrary, and in spite of the fact that anisotropy must be taken into account in the description of many physical phenomena (see [8]), anisotropic Willmore functional has received so far less attention. One reason why research has not developed in this direction as fast as in the isotropic case, lies in the difficulties related to the mathematical treatment of anisotropy.

First steps in the study of an anisotropic version of (1.1) can be found in [5]. Here the author, motivated by surface restoration problems, investigates the problem of finding a fourth order energy having Wulff shapes as minimizers. The functional studied by Clarenz (see also [14]) is obtained by replacing $h$ in (1.1) with its anisotropic version $h_{\gamma}$ (see (2.8) below). In [9] Diewald provides a finite element discretization of the related gradient flow: however extremely few numerical tests in the anisotropic setting are presented. On the other hand some numerical experiments for the related anisotropic elastic flow of curves are shown in [1, $\S 6.2]$. These flows are interesting in their own 
right but they are not geometrical, in the sense that typical features of the isotropic setting are not reproduced in the anisotropic setting, unless some ad-hoc mobility factor is incorporated in the equation for the flow (see Remark 3.2 below).

On the other hand the author has provided and motivated in [18] a geometrically consistent formulation of anisotropic Willmore functional (cf. also [2]). The point of view presented in [18] is the same we adopt in this work. The considered anisotropic Willmore functional (see (2.10) below) now differs from the one studied by Clarenz [5] and Palmer [14] in that the area element $d V$ is replaced by its more natural anisotropic counterpart. The gradient flow is realized by carefully taking into consideration the anisotropic nature of space (more details are given in Section 2 and in Section 3 below). With this approach relevant features of the isotropic setting carry over to the anisotropic one (see for instance Example 3.1 below). In this paper we provide a mixed numerical scheme for the evolution of parametric hypersurfaces, that uses piecewise linear finite elements and that treats nonlinearities in an explicit way. The way the scheme is derived yields an immediate stability result for its semi-discrete formulation. Experimental stability of the fully discrete scheme is achieved through the introduction of a carefully chosen stability term. Along with other interesting tests we show an appealing numerical example of evolution towards a stationary anisotropic torus (see Figure 14). This is especially intriguing since, to our knowledge, there are very few examples of toroidal anisotropic Willmore surfaces in the literature (cf. also [14], where an example of toroidal critical surface for the considered functional is given under the assumption that the Wulff shape is a surface of revolution). Several tests and simulations are provided also for the evolution of curves: note that further examples for the anisotropic elastic flow of curves can be found in [15], where a variational time discretization of the flow combined with a spatial discretization via piecewise affine finite elements is studied.

This work is organized as follows. In Section 2 we briefly review the definitions of anisotropic mean curvature vector, anisotropic area functional and anisotropic Willmore functional. Furthermore we recall some important results concerning the first variation for the anisotropic Willmore functional. In Section 3 we provide a geometrically meaningful formulation of Willmore flow. Due to the highly nonlinear character of the flow we explore for possibilities of geometrically equivalent flows (i.e. equivalent up to tangential components) that might be easier to treat numerically. In Section 4 we compute the first variation of the anisotropic Willmore functional and provide a variational formulation of the flows described in Section 3: here we pay particular attention to allow only for those operations (specifically, integration by parts) that can be carried out also in the spatial discrete setting, so that eventually it will be easy to infer a decrease of the energy for the semi-discrete schemes (stability). After having chosen a formulation of the flow most suitable for numerical computation its discretization in space is discussed in Section 5. Fully discrete semi-implicit schemes and numerical tests are presented in Section 6: here successful ideas for experimentally stable fully discrete schemes are developed first in the curve setting, where we can carry out some elementary analysis and heuristics that help identifying good discretization strategies. After presenting in Section 6.1.1 several numerical tests for the anisotropic Willmore flow of curves, a discretization scheme is provided for the surface setting. Simulations of anisotropic Willmore flow for surfaces are collected in Section 6.2.1.

\section{Preliminaries}

Here we briefly recall some notation and results that appeared in [5] and [18] and that are relevant to our discussion. 
Let $\mathbf{x}: \tilde{\Gamma} \rightarrow \mathbb{R}^{n}$ be a smooth immersion of a smooth oriented compact hypersurface $\tilde{\Gamma}$ (without boundary) of $\mathbb{R}^{n}$. The induced metric on $\tilde{\Gamma}$ is given by

$$
g(v, w)=D \mathbf{x}(v) \cdot D \mathbf{x}(w) \quad \forall v, w \in T_{p} \tilde{\Gamma}
$$

where $\cdot$ is the Euclidean inner product. The differential of the normal mapping $v: \tilde{\Gamma} \rightarrow S^{n-1}$ induces the shape operator $S: T_{p} \tilde{\Gamma} \rightarrow T_{p} \tilde{\Gamma}$ via $D \mathbf{x} \circ S=D v$.

The anisotropic area functional is given by

$$
A_{\gamma}(\mathbf{x}):=\int_{\tilde{\Gamma}} \gamma(v) d V=\int_{\tilde{\Gamma}} d \mu,
$$

where $d V$ is the induced area element and the weighting function $\gamma: \mathbb{R}^{n} \rightarrow[0, \infty)$ is a norm on $\mathbb{R}^{n}$. We assume that $\gamma$ is as smooth as required (at least $\gamma \in C^{2}\left(\mathbb{R}^{n} \backslash\{0\}\right)$ for the definitions of curvature that follow, but more will be required later on). If we identify the normal $v$ with the tangent space of the hypersurface, we can think of the anisotropic map $\gamma$ as assigning different weights to different tangent spaces. When $\gamma$ is the Euclidean norm, then $\gamma(v) \equiv 1$ and we recover the familiar area definition (isotropic case). We denote by $d \mu$ the anisotropic area element $d \mu=\gamma(\nu) d V$.

Recall that from the homogeneity property of the norm $\gamma$ (that is $\gamma(\lambda x)=|\lambda| \gamma(x)$ for $\mathbb{R} \ni \lambda \neq$ 0 and $x \in \mathbb{R}^{n}$ ) one easily infers that

$$
\begin{aligned}
\gamma^{\prime}(x) \cdot x & =\gamma(x), & \gamma^{\prime}(\lambda x) & =\frac{\lambda}{|\lambda|} \gamma^{\prime}(x), \\
\gamma^{\prime \prime}(x) x \cdot y & =0, & \gamma^{\prime \prime}(\lambda x) & =\frac{1}{|\lambda|} \gamma^{\prime \prime}(x),
\end{aligned}
$$

hold for all $x \in \mathbb{R}^{n} \backslash\{0\}, y \in \mathbb{R}^{n}$ and $\lambda \neq 0$ (cf. [8, § 8.1]).

From now on we assume that $\gamma$ satisfy the ellipticity condition

$$
\gamma^{\prime \prime}(p) q \cdot q \geqslant c_{0}|q|^{2} \quad \forall p, q \in \mathbb{R}^{n}, \quad|p|=1, \quad q \cdot p=0 .
$$

Here $|\cdot|$ is the Euclidean norm and $c_{0}$ is a positive constant.

After the usual identification of $\mathbb{R}^{n}$ with its dual $\left(\mathbb{R}^{n}\right)^{*}$ (cf. $[19, \S 0$ and $\S 1]$ ), we call $I$ the convex body in $\mathbb{R}^{n}$ whose support function is $\gamma: \mathbb{R}^{n} \simeq\left(\mathbb{R}^{n}\right)^{*} \rightarrow[0, \infty)$, i.e.

$$
I=\left\{x \in \mathbb{R}^{n} \mid \quad \xi \cdot x \leqslant \gamma(\xi) \quad \forall \xi \in \mathbb{R}^{n}\right\}
$$

(cf. $[19, \S 2.2])$. In the literature $I$ is often referred to as the Wulff shape and it is known to be the solution of an isoperimetric problem, namely, its boundary minimizes the anisotropic area functional $A_{\gamma}$ in the class of surfaces enclosing the same volume (see for instance [6] and references therein for more details). Let $\gamma^{*}$ be the norm in $\mathbb{R}^{n}$ whose unit ball is $I$, thus

$$
\gamma^{*}(x)=\sup \left\{x \cdot y \mid y \in \mathbb{R}^{n}, \gamma(y) \leqslant 1\right\}, \quad\left(x \in \mathbb{R}^{n}\right),
$$

(or, in other words, $\gamma^{*}$ is dual to $\gamma$ ), and let $T:\left(\mathbb{R}^{n}, \gamma^{*}\right) \rightarrow\left(\left(\mathbb{R}^{n}\right)^{*}, \gamma\right)$ be the duality map $T(u)=\frac{1}{2} \partial\left(\gamma^{*}(u)^{2}\right)$, obtained by considering the subdifferential of the squared norm $\gamma^{*}$ (cf. [18] and references therein). Condition (2.5) ensures that the Banach space $X=\left(\mathbb{R}^{n}, \gamma^{*}\right)$ and its dual space $X^{*}=\left(\left(\mathbb{R}^{n}\right)^{*}, \gamma\right)$ are uniformly convex (see [18, § 2]), so that by [21, Proposition 47.19] we 
can deduce that the duality map $T$ is an odd single-valued bijective continuous map, with $T(0)=0$, $T(\xi)=\gamma^{*}(\xi)\left(\gamma^{*}\right)^{\prime}(\xi)$ for $\xi \neq 0$, and such that its inverse $T^{-1}(p)=\gamma(p) \gamma^{\prime}(p)$ for $p \neq 0$, $T^{-1}(0)=0$ is also continuous and equal to the duality map from $X^{*}$ to $X^{* *}=X$. Moreover one has that $\langle T(\xi), \xi\rangle=\gamma^{*}(\xi)^{2}=\gamma(T(\xi))^{2}$ and $\gamma\left(\left(\gamma^{*}\right)^{\prime}(\xi)\right)=1=\gamma^{*}\left(\gamma^{\prime}(x)\right)$ for $\xi, x \neq 0$ (cf. also [3]). These properties will prove useful in the definition of the flow (see also [18, $\S 5]$ ).

As done in the line above, the pairing of an element from a Banach space with one from its dual will be usually denoted by $\langle$,$\rangle . Also note that throughout this paper we will often identify an$ element $f \in\left(\left(\mathbb{R}^{n}\right)^{*}, \gamma\right)$ with $x_{f} \in \mathbb{R}^{n}$ uniquely defined by $\langle f, y\rangle=x_{f} \cdot y$ for all $y \in \mathbb{R}^{n}$, and we will use the same notation for the two entities.

We denote by $a_{\gamma}: T_{p} \tilde{\Gamma} \rightarrow T_{p} \tilde{\Gamma}$ the symmetric mapping given by

$$
a_{\gamma}=(D \mathbf{x})^{-1} \gamma^{\prime \prime}(\nu) D \mathbf{x} \text {. }
$$

Recall that $a_{\gamma}$ is well defined since $\gamma^{\prime \prime}(v) v=0$ due to (2.4).

Moreover, let $\operatorname{grad}(\cdot)$ and $\operatorname{div}(\cdot)$ denote respectively the gradient of smooth functions on $\tilde{\Gamma}$ and divergence of possibly non-tangential vector fields on $\tilde{\Gamma}$ (for definitions of the operators $\operatorname{grad}(\cdot)$, $\operatorname{div}(\cdot), \operatorname{tr}(\cdot)$ and their representation in local coordinates see for instance $[18, \S 6.1]$ and references given in there). The scalar anisotropic mean curvature is given by

$$
h_{\gamma}:=\operatorname{div}\left(\gamma^{\prime}(v)\right)=\operatorname{tr}\left(a_{\gamma} \circ S\right)
$$

(note that here the definition of (anisotropic) mean curvature does not involve any arithmetic mean), whereas the anisotropic mean curvature vector is given by

$$
\kappa_{\gamma}:=-h_{\gamma} \gamma^{\prime}(v) \text {. }
$$

As in $[5,18]$, we write $\triangle_{\gamma}(\cdot)=\operatorname{div}\left(a_{\gamma} \operatorname{grad}(\cdot)\right)$ and $|S|_{\gamma}^{2}=\operatorname{tr}\left(a_{\gamma} S^{2}\right)$.

Motivated by analytical and geometrical considerations presented by the author in $[18, \S 6]$ a natural and geometrically consistent definition for the anisotropic Willmore functional is given by

$$
W_{\gamma}(\mathbf{x}):=\frac{1}{2} \int_{\tilde{\Gamma}} h_{\gamma}^{2} \gamma(v) d V=\frac{1}{2} \int_{\tilde{\Gamma}} \gamma^{*}\left(\kappa_{\gamma}\right)^{2} d \mu .
$$

Note that $\left|h_{\gamma}\right|$ is the length of the anisotropic mean curvature vector $\kappa_{\gamma}=-h_{\gamma} \gamma^{\prime}(v)$ with respect to the norm $\gamma^{*}$, and recall that $d \mu=\gamma(\nu) d V$ is the anisotropic area element.

REMARK 2.1 It is well known ( [20, Theorem 7.3.1]) that for $n=3$ the classical Willmore functional (i.e. in the isotropic setting) is invariant under conformal transformation of $\mathbb{R}^{3}$. Thus similarity transformation and inversions do not change the functional. In the anisotropic setting the functional (2.10) is still invariant under dilations. Indeed, let $G: \mathbb{R}^{3} \rightarrow \mathbb{R}^{3}$ be defined through $G(x)=\alpha x$ for some fixed $\alpha>0$ and let $\tilde{\mathbf{x}}: \tilde{\Gamma} \rightarrow \mathbb{R}^{3}$ be defined by $\tilde{\mathbf{x}}=G \circ \mathbf{x}$. With $\left\{\partial_{1}, \partial_{2}\right\}$ a local basis for the tangent space $T_{p}(\tilde{\Gamma})$, and $g_{i j}=D \mathbf{x}\left(\partial_{i}\right) \cdot D \mathbf{x}\left(\partial_{i}\right), \tilde{g}_{i j}=D \tilde{\mathbf{x}}\left(\partial_{i}\right) \cdot D \tilde{\mathbf{x}}\left(\partial_{i}\right)$ we get $\alpha^{2} g_{i j}=\tilde{g}_{i j}, d \tilde{V}=\alpha^{2} d V, \gamma(\nu)=\gamma(\tilde{v})$ and $h_{\gamma}=\operatorname{div}\left(\gamma^{\prime}(v)\right)=g^{i j} D\left(\gamma^{\prime}(v)\right)\left(\partial_{i}\right) \cdot D \mathbf{x}\left(\partial_{j}\right)=$ $\alpha \tilde{g}^{i j} D\left(\gamma^{\prime}(\tilde{v})\right)\left(\partial_{i}\right) \cdot D \tilde{\mathbf{x}}\left(\partial_{j}\right)=\alpha \tilde{h}_{\gamma}$, so that $W_{\gamma}(\mathbf{x})=W_{\gamma}(\tilde{\mathbf{x}})$ (here we have used the convention to sum over repeated indices). Further invariances are subject to additional properties of the anisotropy map $\gamma$. For instance if $\gamma$ is rotationally symmetric around the z-axes, then (2.10) is invariant under rotations around this axes.

The first variation for (2.10) is computed in [18]; precisely we have the following result. 
LEMma 2.2 Consider a family of smooth immersions $\mathbf{x}: \tilde{\Gamma} \times\left(-t_{0}, t_{0}\right) \rightarrow \mathbb{R}^{n}$, such that $\mathbf{x}(\cdot, 0)=\mathbf{x}$ and

$$
\left.\partial_{t}\right|_{t=0} \mathbf{x}(\cdot, t)=\varphi v+D \mathbf{x}(v)=: \theta
$$

where $\varphi$ is a smooth function and $v$ is a smooth tangential vector field on $\tilde{\Gamma}$. We have that

$$
\begin{aligned}
\left.\frac{d}{d t}\right|_{t=0} & W_{\gamma}(\mathbf{x}(t))=\left\langle W_{\gamma}^{\prime}(\mathbf{x}), \theta\right\rangle \\
& =\int_{\tilde{\Gamma}} \theta \cdot\left(\frac{1}{2} h_{\gamma}^{3}-\gamma(v) \triangle_{\gamma} h_{\gamma}-h_{\gamma} \operatorname{tr}\left(\left(a_{\gamma} \circ S\right)^{2}\right)-2 g\left(\operatorname{grad} h_{\gamma}, a_{\gamma} \operatorname{grad} \gamma(v)\right)\right) v d V .
\end{aligned}
$$

REMARK 2.3 (i) For $n=2$ one can show that Wulff shapes, more precisely the boundary of the convex body $I$ and any rescaled/translated version of it, are critical points for $W_{\gamma}$ subject to fixed anisotropic length (see [18, Lemma 6.5]).

(ii) For $n=3$ Wulff shapes are critical points for $W_{\gamma}$ (see [18, Lemma 6.4]).

The statement (ii) above can be strengthened as follows.

THEOREM 2.4 Let $\gamma: \mathbb{R}^{3} \rightarrow[0, \infty)$ be a smooth norm satisfying (2.5) and $\mathbf{x}: \tilde{\Gamma} \rightarrow \mathbb{R}^{3}$ a smooth immersion of a compact oriented two-dimensional surface without boundary. We have that

$$
W_{\gamma}(\mathbf{x}) \geqslant 2 A_{\gamma}(\partial I)
$$

where $I$ is the Wulff shape (2.6). Wulff shapes are the unique minimizers for the anisotropic Willmore functional $W_{\gamma}$.

Proof. A proof of the isotropic case can be found in [20, Theorem 7.2.2]. The proof in the anisotropic setting uses exactly the same arguments of the analogous [5, Theorem 4.1], the main difference being that in [5] the author considers the functional $\int_{\tilde{\Gamma}} h_{\gamma}^{2} d V$ and relates it to the isotropic area functional. Here we report all arguments for the sake of completeness.

First of all observe that because of (2.5) the anisotropic mean curvature may be written as

$$
h_{\gamma}=\operatorname{tr}\left(a_{\gamma} \circ S\right)=\operatorname{tr}\left(a_{\gamma}^{\frac{1}{2}} \circ S \circ a_{\gamma}^{\frac{1}{2}}\right) .
$$

The symmetric endomorphism field $a_{\gamma}^{\frac{1}{2}} \circ S \circ a_{\gamma}^{\frac{1}{2}}$ may be diagonalized with eigenvalues $\tilde{\mu}_{1}$ and $\tilde{\mu}_{2}$. Let $K_{\gamma}$ be the anisotropic Gauss curvature $K_{\gamma}:=\operatorname{det}\left(a_{\gamma} \circ S\right)=\operatorname{det}\left(a_{\gamma}^{\frac{1}{2}} \circ S \circ a_{\gamma}^{\frac{1}{2}}\right)$. Using the fact that $h_{\gamma}^{2}-4 K_{\gamma}=\left(\tilde{\mu}_{1}+\tilde{\mu}_{2}\right)^{2}-4 \tilde{\mu}_{1} \tilde{\mu}_{2}=\left(\tilde{\mu}_{1}-\tilde{\mu}_{2}\right)^{2} \geqslant 0$ we deduce that

$$
W_{\gamma}(\mathbf{x}) \geqslant \frac{1}{2} \int_{K^{+}} h_{\gamma}^{2} d \mu \geqslant 2 \int_{K^{+}} K_{\gamma} d \mu,
$$

where $K^{+}=\{\xi \in \tilde{\Gamma}: K(\xi) \geqslant 0\}$ with $K$ the classical Gauss curvature. Since the surface is compact we get in every normal direction a point $\xi$ where $K(\xi) \geqslant 0$, thus $K^{+} \neq \emptyset$ and $v\left(K^{+}\right)=S^{2}$ (cf. for instance [20, § 7.2]).

On the other hand notice that the map $\gamma^{\prime}: S^{2} \rightarrow \mathbb{R}^{3}, v \mapsto \gamma^{\prime}(v)$, provides a parametrization for the boundary of the Wulff shape $I$ (recall $\gamma^{*}\left(\gamma^{\prime}(v)\right)=1$ and $\left.\gamma^{\prime \prime}(v)\right|_{\nu \perp}$ is positive definite due to (2.5)). Moreover for the normal mapping $v: \tilde{\Gamma} \rightarrow S^{2}$ observe that the normal $v(\xi)$ at a point 
$\xi \in \tilde{\Gamma}$ coincides with the normal $v(p)$ at the point $p=\gamma^{\prime}(v(\xi)) \in \partial I$. Next consider a local chart $\varphi: \Omega \subset \mathbb{R}^{2} \rightarrow \tilde{\Gamma}$ for $\xi \in \tilde{\Gamma}$ such that $\gamma^{\prime}(\nu \circ \varphi)$ is also a local chart for $p=\gamma^{\prime}(\nu(\xi)) \in \partial I$. Then

$$
\partial_{i}\left(\gamma^{\prime}(\nu \circ \varphi)\right)=\frac{\partial}{\partial x_{i}}\left(\gamma^{\prime} \circ v \circ \mathbf{x}^{-1} \circ \mathbf{x} \circ \varphi\right)=\gamma^{\prime \prime}(v) D\left(v \circ \mathbf{x}^{-1}\right) \frac{\partial}{\partial x_{i}}(\mathbf{x} \circ \varphi) \quad i=1,2,
$$

and we can relate the area elements for $\tilde{\Gamma}$ and $\partial I$ as follows

$$
\begin{aligned}
\left|\partial_{1}\left(\gamma^{\prime}(v \circ \varphi)\right) \wedge \partial_{2}\left(\gamma^{\prime}(\nu \circ \varphi)\right)\right| & =\left|\operatorname{det}\left(\gamma^{\prime \prime}(v) D\left(v \circ \mathbf{x}^{-1}\right)\right)\right|\left|\frac{\partial}{\partial x_{1}}(\mathbf{x} \circ \varphi) \wedge \frac{\partial}{\partial x_{2}}(\mathbf{x} \circ \varphi)\right| \\
& =\left|K_{\gamma}\right|\left|\frac{\partial}{\partial x_{1}}(\mathbf{x} \circ \varphi) \wedge \frac{\partial}{\partial x_{2}}(\mathbf{x} \circ \varphi)\right| .
\end{aligned}
$$

It follows that $2 \int_{K^{+}} K_{\gamma} d \mu, \geqslant 2 A_{\gamma}(\partial I)$.

Next suppose that $W_{\gamma}(\mathbf{x})=2 A_{\gamma}(\partial I)$. Then it must be $\tilde{\mu}_{1}=\tilde{\mu}_{2}=: \tilde{\mu}$ and thus also $a_{\gamma}^{\frac{1}{2}} \circ S \circ$ $a_{\gamma}^{\frac{1}{2}}=\tilde{\mu} I d$. It follows that $S \circ a_{\gamma}=\tilde{\mu} I d$. As in [5] one derives $\operatorname{grad}(\tilde{\mu})=\operatorname{div}\left(S \circ a_{\gamma}\right)=\operatorname{grad}\left(h_{\gamma}\right)$, from which it follows that $h_{\gamma}=\tilde{\mu}+$ const. on $\tilde{\Gamma}$. Since $h_{\gamma}=\operatorname{tr}\left(a_{\gamma} \circ S\right)=\operatorname{tr}(\tilde{\mu} I d)=2 \tilde{\mu}$, we finally get that $\tilde{\mu}=$ const. $\neq 0$ on $\tilde{\Gamma}$. Next, from $a_{\gamma} \circ S=\tilde{\mu} I d$ we infer $D\left(\gamma^{\prime}(v)\right)=\gamma^{\prime \prime}(v) D v=$ $\tilde{\mu} D \mathbf{x}$, which leads to $\mathbf{x}=x_{0}+\frac{1}{\tilde{\mu}} \gamma^{\prime}(v)$ with $x_{0}$ a constant vector in $\mathbb{R}^{3}$, and the claim follows.

Notation and general assumptions outlined in this section will be used throughout this work.

\section{Anisotropic Willmore flow}

To define the anisotropic Willmore flow we follows ideas presented in [18] for the anisotropic mean curvature flow. A key point is to take into account the anisotropic nature of space and encode this information in the formulation of the flow. When this is done correctly we observe that properties typical of the isotropic case extend naturally to the anisotropic setting: an example is given in Example 3.1 below.

DEFINITION 3.1 Let $\mathbf{x}_{0}: \tilde{\Gamma} \rightarrow \mathbb{R}^{n}$ be an isometric immersion. The anisotropic Willmore flow is a family of immersions $\mathbf{x}:[0, \bar{T}) \times \tilde{\Gamma} \rightarrow \mathbb{R}^{n}$ parametrized by $t$ such that $\mathbf{x}(0)=\mathbf{x}_{0}$ and

$$
\int_{\tilde{\Gamma}}\left\langle T\left(\partial_{t} \mathbf{x}\right), \theta\right\rangle \gamma(v) d V(t)=-\left\langle W_{\gamma}^{\prime}(\mathbf{x}(t)), \theta\right\rangle
$$

for all test functions $\theta \in C^{\infty}\left(\tilde{\Gamma}, \mathbb{R}^{n}\right)$ and $t \in(0, \bar{T})$.

Note that we have used the duality map $T$ to relate in a consistent way the velocity vector $\partial_{t} \mathbf{x}$ to the gradient given by the first variation of the anisotropic Willmore functional (cf. [18, $\S 4]$ for details on functional analytic aspects). Recall that in the isotropic setting (where $\gamma$ is simply the Euclidean norm) $\left\langle T\left(\partial_{t} \mathbf{x}\right), \theta\right\rangle=\partial_{t} \mathbf{x} \cdot \theta$ and thus we recover the familiar formulation of $L^{2}$-gradient flow.

The anisotropic Willmore energy decreases along the flow since due to the property of the duality $\operatorname{map}\langle T(\xi), \xi\rangle=\gamma^{*}(\xi)^{2}$ we have that

$$
\begin{aligned}
\frac{d}{d t} W_{\gamma}(\mathbf{x}(t)) & =\left\langle W_{\gamma}^{\prime}(\mathbf{x}(t)), \partial_{t} \mathbf{x}\right\rangle \\
& =-\int_{\tilde{\Gamma}}\left\langle T\left(\partial_{t} \mathbf{x}\right), \partial_{t} \mathbf{x}\right\rangle \gamma(v) d V(t)=-\int_{\tilde{\Gamma}} \gamma^{*}\left(\partial_{t} \mathbf{x}\right)^{2} \gamma(v) d V(t) \leqslant 0 .
\end{aligned}
$$


After the usual identification of $\mathbb{R}^{n}$ with its dual $\left(\mathbb{R}^{n}\right)^{*}$ and recalling (2.12), a classical formulation for the flow is given by

$$
T\left(\partial_{t} \mathbf{x}\right)=-\left(\frac{1}{2} h_{\gamma}^{3}-\gamma(v) \triangle_{\gamma} h_{\gamma}-h_{\gamma} t r\left(\left(a_{\gamma} \circ S\right)^{2}\right)-2 g\left(\operatorname{grad} h_{\gamma}, a_{\gamma} \operatorname{grad} \gamma(v)\right)\right) \frac{v}{\gamma(v)} .
$$

Applying $T^{-1}$ to both sides we obtain

$$
\partial_{t} \mathbf{x}=-\left(\frac{1}{2} h_{\gamma}^{3}-\gamma(v) \triangle_{\gamma} h_{\gamma}-h_{\gamma} \operatorname{tr}\left(\left(a_{\gamma} \circ S\right)^{2}\right)-2 g\left(\operatorname{grad} h_{\gamma}, a_{\gamma} \operatorname{grad} \gamma(v)\right)\right) \gamma^{\prime}(v) .
$$

EXAMPLE 3.1 In the planar case Wulff shapes expand with the same velocity as do circles in the isotropic setting. Indeed if we consider the parametrization $\mathbf{x}:[0, \bar{T}) \times S^{1} \rightarrow \mathbb{R}^{2}, \mathbf{x}(t, \theta)=$ $R(t) \gamma^{\prime}(\cos \theta, \sin \theta)$, then since $h_{\gamma}=\frac{1}{R(t)}$ and $\operatorname{tr}\left(\left(a_{\gamma} \circ S\right)^{2}\right)=\frac{1}{R(t)^{2}}$ (see for instance [18, $\left.\S 5.3 .1\right]$ ) equation (3.2) implies

$$
\frac{d}{d t} R(t)=\frac{1}{2 R(t)^{3}}
$$

which is solved by $R(t)=(R(0)+2 t)^{\frac{1}{4}}$ as in the isotropic case.

In contrast to the above example we make the following remark.

REMARK 3.2 In [5] a slightly different version of anisotropic Willmore functional is studied, namely

$$
\tilde{W}_{\gamma}(\mathbf{x})=\frac{1}{2} \int_{\tilde{\Gamma}} h_{\gamma}^{2} d V .
$$

The above functional differs from (2.10) in that the anisotropic area element is replaced by the isotropic one. For curves $\mathbf{x}:[0, \bar{T}) \times S^{1} \rightarrow \mathbb{R}^{2}, \mathbf{x}=\mathbf{x}(t, \theta)$, with isotropic length element $d s=$ $\left|\mathbf{x}_{\theta}\right| d \theta$, the $L^{2}$-gradient flow

$$
\int_{S^{1}} \frac{\mathbf{x}_{t}}{m(v)} \cdot \psi d s=-\left\langle\tilde{W}_{\gamma}^{\prime}(\mathbf{x}(t)), \psi\right\rangle \quad \forall \psi \in C^{\infty}\left(S^{1}, \mathbb{R}^{2}\right)
$$

with positive mobility factor $m: S^{1} \rightarrow \mathbb{R}$ induces the evolution equation

$$
\frac{\mathbf{x}_{t}}{m(v)}=\left(\triangle_{\gamma} h_{\gamma}+h_{\gamma}|S|_{\gamma}^{2}-\frac{1}{2} h h_{\gamma}^{2}\right) v
$$

whose normal velocity $V=\mathbf{x}_{t} \cdot v$ satisfies

$$
\frac{V}{m(v)}=\triangle_{\gamma} h_{\gamma}+h_{\gamma}|S|_{\gamma}^{2}-\frac{1}{2} h h_{\gamma}^{2}
$$

(cf. computations for the first variation of $\tilde{W}_{\gamma}$ given in [5]). Since for curves $|S|_{\gamma}^{2}=\operatorname{tr}\left(a_{\gamma} S^{2}\right)=$ $h h_{\gamma}$, a straightforward computation shows that a Wulff shape $\mathbf{x}:[0, \bar{T}) \times S^{1} \rightarrow \mathbb{R}^{2}, \mathbf{x}(t, \theta)=$ $(R(0)+2 t)^{1 / 4} \gamma^{\prime}(\cos \theta, \sin \theta)$ satisfies (3.3) provided $m(v)=\gamma(v)\left(\gamma^{\prime \prime}(\nu) \tau \cdot \tau\right)$ where $v=$ $(\cos \theta, \sin \theta)$ and $\tau=(\sin \theta,-\cos \theta)$ (cf. also [1, Theorem 2.1]).

Goal of this paper is to find a suitable FEM-discretization of the flow that uses piecewise linear finite elements. With this in mind, a mixed formulation for the flow of Definition 3.1 is the following. 
Problem 3.3 Given an initial isometric immersion of $\mathbf{x}_{0}: \tilde{\Gamma} \rightarrow \mathbb{R}^{n}$, find $\mathbf{x}, \kappa_{\gamma}:[0, \bar{T}) \times \tilde{\Gamma} \rightarrow \mathbb{R}^{n}$ such that $\mathbf{x}(0)=\mathbf{x}_{0}, \kappa_{\gamma}(0)=-\left(h_{\gamma} \gamma^{\prime}(v)\right)(0)$, and such that on $(0, \bar{T})$ the following holds

$$
\begin{array}{cc}
\int_{\tilde{\Gamma}}\left\langle T\left(\partial_{t} \mathbf{x}\right), \theta\right\rangle \gamma(v) d V(t)=-\left\langle W_{\gamma}^{\prime}(\mathbf{x}(t)), \theta\right\rangle & \forall \theta \in C^{\infty}\left(\tilde{\Gamma}, \mathbb{R}^{n}\right), \\
\int_{\tilde{\Gamma}}\left\langle T\left(\kappa_{\gamma}\right), \varphi\right\rangle \gamma(v) d V(t)=-\left\langle A_{\gamma}^{\prime}(\mathbf{x}(t)), \varphi\right\rangle & \forall \varphi \in C^{\infty}\left(\tilde{\Gamma}, \mathbb{R}^{n}\right) .
\end{array}
$$

To justify the term "mixed formulation", it will be shown below (see Lemma 4.11) that the righthand side of equation (3.4) contains indeed $\kappa_{\gamma}$. Note that (3.5) properly defines $\kappa_{\gamma}$ in weak terms since the first variation for the anisotropic area functional is given by

$$
\left\langle A_{\gamma}^{\prime}(\mathbf{x}), \varphi\right\rangle=\int_{\tilde{\Gamma}} h_{\gamma} v \cdot \varphi d V \quad \forall \varphi \in C^{\infty}\left(\tilde{\Gamma}, \mathbb{R}^{n}\right),
$$

(see for example [4] and [8, § 8.3] or Remark 4.9 below) and $\kappa_{\gamma}=-h_{\gamma} \gamma^{\prime}(v)=T^{-1}\left(-h_{\gamma} \frac{v}{\gamma(v)}\right)$ (recall (2.9)).

\subsection{An alternative formulation through a geometrically equivalent flow}

The flow described in Problem 3.3 is rather hard to treat numerically because it is highly nonlinear. For a discretization with finite elements the presence of the nonlinear operator $T$ on both the time derivative and curvature vector poses an additional problem. In principle one could try working with approximations of the terms $T\left(\partial_{t} \mathbf{x}\right)$ and $T\left(\kappa_{\gamma}\right)$ via Taylor expansions. However we are faced with several challenges. First of all, since we expect $\partial_{t} \mathbf{x}$ to tend to zero, it would make sense to consider a linearization of $T$ around the origin. However, although $T$ is well defined in zero $(T(0)=0)$, in general, it will not be differentiable here (except for special cases. For instance if $\gamma^{*}(\xi)=\sqrt{A \xi \cdot \xi}$ where $A$ is a positive definite symmetric matrix, then $T$ is linear with $T \xi=A \xi$ and of course everywhere differentiable. Cf. also comments in the proof of Lemma 4.11 below.) Secondly, even if we tried to use the identity $T(\xi)=T^{\prime}(\xi) \xi$ for $\xi \neq 0$ (which follows from the positive homogeneity of $T$, namely $T(a \xi)=a T(\xi)$ for $a>0)$, we would still have a numerical scheme that involves computations of $\gamma^{*}, \gamma$, as well as their derivatives. This is a situation that we would gladly avoid.

To get around these problems and in particular to "get rid of" the nonlinear operator $T$ acting on the time derivative $\partial_{t} \mathbf{x}$ and on $\kappa_{\gamma}$ we will eventually consider another flow which, up to tangential components, is equivalent to (3.2). Precisely, let us consider

$$
\frac{1}{\gamma(v)} \partial_{t} \mathbf{x}=-\left(\frac{1}{2} h_{\gamma}^{3}-\gamma(v) \Delta_{\gamma} h_{\gamma}-h_{\gamma} t r\left(\left(a_{\gamma} \circ S\right)^{2}\right)-2 g\left(\operatorname{grad} h_{\gamma}, a_{\gamma} \operatorname{grad} \gamma(v)\right)\right) v,
$$

which is obtained by taking the normal component in the righthand-side of (3.2). In weak form this flow is described by

$$
\int_{\tilde{\Gamma}} \frac{1}{\gamma(v)} \partial_{t} \mathbf{x} \cdot \theta d V(t)=-\left\langle W_{\gamma}^{\prime}(\mathbf{x}(t)), \theta\right\rangle \quad \forall \theta \in C^{\infty}\left(\tilde{\Gamma}, \mathbb{R}^{n}\right) .
$$

Note that by setting

$$
\kappa_{\gamma}^{N}:=\kappa_{\gamma} \cdot v v=-\gamma(v) h_{\gamma} v
$$


we can write $W_{\gamma}(\mathbf{x})=\frac{1}{2} \int_{\tilde{\Gamma}}\left|\frac{k_{\nu}^{N}}{\gamma(v)}\right|^{2} \gamma(\nu) d V$. Moreover, because of (3.6), a weak formulation for $k_{\gamma}^{N}$ is given by

$$
\int_{\tilde{\Gamma}} k_{\gamma}^{N} \cdot \varphi \frac{1}{\gamma(\nu)} d V=-\left\langle A_{\gamma}^{\prime}(\mathbf{x}), \varphi\right\rangle \quad \forall \varphi \in C^{\infty}\left(\tilde{\Gamma}, \mathbb{R}^{n}\right) .
$$

Therefore a weak mixed formulation for the flow (3.7) can be formulated as follows.

Problem 3.4 Given an initial isometric immersion $\mathbf{x}_{0}: \tilde{\Gamma} \rightarrow \mathbb{R}^{n}$ find $\mathbf{x}, \kappa_{\gamma}^{N}:[0, \bar{T}) \times \tilde{\Gamma} \rightarrow \mathbb{R}^{n}$ such that $\mathbf{x}(0)=\mathbf{x}_{0}, \kappa_{\gamma}^{N}(0)=-\left(h_{\gamma} \gamma(v) v\right)(0)$, and such that on $(0, \bar{T})$ the following holds

$$
\begin{array}{ll}
\int_{\tilde{\Gamma}} \frac{1}{\gamma(v)} \partial_{t} \mathbf{x} \cdot \theta d V(t)=-\left\langle W_{\gamma}^{\prime}(\mathbf{x}(t)), \theta\right\rangle & \forall \theta \in C^{\infty}\left(\tilde{\Gamma}, \mathbb{R}^{n}\right), \\
\int_{\tilde{\Gamma}} \frac{1}{\gamma(v)} \kappa_{\gamma}^{N} \cdot \varphi d V(t)=-\left\langle A_{\gamma}^{\prime}(\mathbf{x}(t)), \varphi\right\rangle \quad \forall \varphi \in C^{\infty}\left(\tilde{\Gamma}, \mathbb{R}^{n}\right) .
\end{array}
$$

It will be shown below (see Lemma 4.12) that the right-hand side of equation (3.11) contains indeed $\kappa_{\gamma}^{N}$.

\section{Variational formulation}

In the following we derive a first variation of the anisotropic Willmore functional that can be carried out also in the (spatial) discrete setting. A nice "side effect" is that it is not hard to prove stability for the semi-discrete problem (this idea was first used in [10]).

A mixed formulation of the Willmore flow and its alternative (as described in Section 3.1), that are suitable for discretization, are provided at the end of this section.

First of all we introduce some necessary notation, define and discuss the relevant anisotropic operators, and provide for these some results and observations that are of independent interest.

\subsection{Set up and notation}

For simplicity we take $\tilde{\Gamma}$ to be embedded in $\mathbb{R}^{n}$ through the identity map and we do not distinguish between $\tilde{\Gamma}$ and the image $\Gamma=\mathbf{x}(\tilde{\Gamma})$. Therefore $\Gamma$ denotes a smooth, compact, oriented hypersurface of $\mathbb{R}^{n}$ without boundary.

Since, to a certain extent, our numerical treatment of the anisotropic Willmore flow can be thought of as a generalization of [10] to the anisotropic setting, from now on we will use most of the notation adopted there. As in [10] it will be convenient to work with the identity map on the surface. Thus, we define $u: \Gamma \rightarrow \mathbb{R}^{n}$ by

$$
u(X(x))=I_{\Gamma}(X(x))=X(x), \quad x \in U
$$

where $X: U \rightarrow \mathbb{R}^{n}, U \subset \mathbb{R}^{n-1}$, is a local parametrisation of $\Gamma$.

4.1.1 General notation. For $a, b \in \mathbb{R}^{n}$ we denote by $a \cdot b$ the Euclidean inner product. For $n \times n$ matrices $A, B$ we write $A B$ for the usual matrix multiplication and

$$
A: B=\sum_{i, j=1}^{n} A_{i j} B_{i j}=\operatorname{tr}\left(A B^{T}\right)=\operatorname{tr}\left(A^{T} B\right)
$$


for the matrix scalar product. Moreover $a \otimes b$ denotes the matrix with entries

$$
(a \otimes b)_{i j}=a_{i} b_{j} .
$$

The gradient of a smooth function $f: \mathbb{R}^{n} \rightarrow \mathbb{R}$ is denoted by $\nabla f(x)=f^{\prime}(x)=$ $\left(\partial_{1} f(x), \ldots, \partial_{n} f(x)\right)=\left(f_{p_{1}}(x), \ldots, f_{p_{n}}(x)\right) \in \mathbb{R}^{n}$.

4.1.2 Isotropic quantities. For a sufficiently smooth $f: \Gamma \rightarrow \mathbb{R}$, defined in a neighbourhood of $\Gamma$, we recall that the tangential gradient on the surface $\Gamma$ with normal $v$ is given by

$$
\nabla_{\Gamma} f=\nabla f-\nabla f \cdot v v,
$$

with spacial components $\nabla_{\Gamma} f=\left(\underline{D}_{1} f, \ldots, \underline{D}_{n} f\right)$. Introducing the projection $P \in \mathbb{R}^{n \times n}$ defined by

$$
P_{i j}=\delta_{i j}-v^{i} v^{j} \quad(i, j=1, \ldots, n),
$$

it is easy to see that $\nabla_{\Gamma} f=P \nabla f$. For a vector field $\varphi: \Gamma \rightarrow \mathbb{R}^{n}, \varphi=\left(\varphi^{1}, \ldots, \varphi^{n}\right)$, the divergence is given by

$$
\nabla_{\Gamma} \cdot \varphi=\sum_{m=1}^{n} \underline{D}_{m} \varphi^{m}=\operatorname{tr}(P \nabla \varphi),
$$

where $(\nabla \varphi)_{i j}=\partial_{j} \varphi^{i}$. Note that for a given smooth $f: \Gamma \rightarrow \mathbb{R}$ it is always possible to find a smooth extension of $f$ in a neighbourhood of $\Gamma$, for instance by using the distance function. The definitions of tangential gradient and divergence do not depend on the extension chosen, see for instance [8]. For the sake of readability we will from now on adopt the convention to sum over repeated indices (so that for example the expression above becomes $\nabla_{\Gamma} \cdot \varphi=\underline{D}_{m} \varphi^{m}$ ). The Laplace Beltrami operator is defined by

$$
\triangle_{\Gamma} f=\nabla_{\Gamma} \cdot \nabla_{\Gamma} f=\underline{D}_{m} \underline{D}_{m} f .
$$

Note that for a given a vector field $\varphi: \Gamma \rightarrow \mathbb{R}^{n}$, the matrix $\nabla_{\Gamma} \varphi$ has entries

$$
\left(\nabla_{\Gamma} \varphi\right)_{i j}=\underline{D}_{j} \varphi^{i} .
$$

(This follows the notation used for the Jacobi matrix of a vector valued function, however it is different from the notation used in [10].) We have that $\nabla_{\Gamma} \varphi=\nabla \varphi P$.

The symmetric matrix $\nabla_{\Gamma} v$, also known as extended Weingarten map, has one zero eigenvalue corresponding to the eigenvector $v$. The remaining eigenvalues are the so called principal curvatures $\kappa_{1}, \ldots \kappa_{n-1}$. The mean curvature is defined by

$$
h=\nabla_{\Gamma} \cdot v=\sum_{i=1}^{n-1} \kappa_{i} .
$$

It is not difficult to show that

$$
-\triangle_{\Gamma} I d=h v \quad \text { on } \Gamma
$$

where $I d: \mathbb{R}^{n} \rightarrow \mathbb{R}^{n}$ is the identity map (cf. for instance $[8, \S 2]$ ). We recall that the formula for integration by parts on the surface $\Gamma$ is

$$
\int_{\Gamma} \underline{D}_{i} f d V=\int_{\Gamma} f h v_{i} d V
$$


where $d V$ denotes the surface volume element ( [8, § 2.4]).

Assuming that $\Gamma$ is locally described by a diffeomorphism $X: U \rightarrow \mathbb{R}^{n}$, where $U$ is an open subset of $\mathbb{R}^{n-1}$, and by using the standard notation $g_{i j}=\partial_{i} X \cdot \partial_{j} X, g=\operatorname{det}\left(g_{i j}\right), g^{i j}=$ $\left(\left(g_{i j}\right)^{-1}\right)_{i j}$, for $i, j=1, \ldots, n-1$, it is easy to infer that

$$
\nabla_{\Gamma} f \circ X=g^{i j} \partial_{j}(f \circ X) \partial_{i} X, \quad \underline{D}_{m} f=g^{i j} \partial_{j}(f \circ X) \partial_{i} X^{m},
$$

$\left(\nabla_{\Gamma} f \cdot \nabla_{\Gamma} h\right) \circ X=g^{i j} \partial_{i}(f \circ X) \partial_{j}(h \circ X), \quad\left(\nabla_{\Gamma} \varphi: \nabla_{\Gamma} \psi\right) \circ X=g^{i j} \partial_{i}(\varphi \circ X) \cdot \partial_{j}(\psi \circ X)$,

where $f, h: \Gamma \rightarrow \mathbb{R}$, and $\varphi, \psi: \Gamma \rightarrow \mathbb{R}^{n}$ are smooth maps.

4.1.3 Anisotropic quantities. As usual let $\gamma: \mathbb{R}^{n} \rightarrow[0, \infty)$ be a sufficiently smooth norm and let $v$ denote the unit vector normal to $\Gamma$. As in [18] we define $P^{\gamma} \in \mathbb{R}^{n \times n}$ to be the matrix

$$
\left(P^{\gamma}\right)_{i j}:=\left(I-\nabla \gamma(v) \otimes \frac{v}{\gamma(v)}\right)_{i j}=\delta_{i j}-\gamma_{p_{i}}(v) \frac{v^{j}}{\gamma(v)} .
$$

Note that $P^{\gamma}$ and its transpose

$$
\tilde{P}^{\gamma}:=\left(P^{\gamma}\right)^{T}=\left(I-\frac{v}{\gamma(v)} \otimes \nabla \gamma(v)\right)
$$

are projectors, that is $\left(P^{\gamma}\right)^{2}=P^{\gamma}$ and $\left(\tilde{P}^{\gamma}\right)^{2}=\tilde{P}^{\gamma}$. For a given smooth map $f: \Gamma \rightarrow \mathbb{R}$, defined in a neighbourhood of $\Gamma$, we define the anisotropic tangential gradient to be

$$
\nabla_{\Gamma}^{\gamma} f:=\nabla f-\nabla f \cdot \nabla \gamma(v) \frac{v}{\gamma(v)}=\tilde{P}^{\gamma} \nabla f,
$$

with spacial components $\nabla_{\Gamma}^{\gamma} f=\left(\underline{D}_{1}^{\gamma} f, \ldots, \underline{D}_{n}^{\gamma} f\right)$. Observe that for $w \in \mathbb{R}^{n}$ we have that $P^{\gamma} w=$ $w-w \cdot \frac{v}{\gamma(v)} \nabla \gamma(v) \in<v>^{\perp}$, whereas $\tilde{P}^{\gamma} w=w-w \cdot \nabla \gamma(v) \frac{v}{\gamma(v)} \in<\nabla \gamma(v)>^{\perp}$.

It is easy to verify that

$$
\begin{array}{ll}
P P^{\gamma}=P^{\gamma}, & \tilde{P}^{\gamma} P=\tilde{P}^{\gamma}, \\
P^{\gamma} P=P, & P \tilde{P}^{\gamma}=P .
\end{array}
$$

In particular we see that $\nabla_{\Gamma}^{\gamma} f=\tilde{P}^{\gamma} P \nabla f=\tilde{P}^{\gamma} \nabla_{\Gamma} f$ and therefore we deduce that the definition given in (4.15) does only depend on the value of $f$ on $\Gamma$ (and not on the possibly chosen extension).

For a vector field $\varphi: \Gamma \rightarrow \mathbb{R}^{n}$, the matrix $\nabla_{\Gamma}^{\gamma} \varphi$ has entries $\left(\nabla_{\Gamma}^{\gamma} \varphi\right)_{i j}=\underline{D}_{j}^{\gamma} \varphi^{i}$. For the identity map $I d(x)=x$ in $\mathbb{R}^{n}$ one immediately deduces that $\underline{D}_{k} x^{j}=\delta_{k j}-v^{k} v^{j}=P_{k j}$ and in the anisotropic setting

$$
\delta_{j k}-\frac{\nu_{k}}{\gamma(\nu)} \gamma_{p_{j}}(\nu)=P_{j k}^{\gamma}=\tilde{P}_{k j}^{\gamma}=\underline{D}_{k}^{\gamma} x^{j}=\left(\nabla_{\Gamma}^{\gamma} I d\right)_{j k},
$$

where $j, k=1, \ldots, n$. For a given a vector field $\varphi: \Gamma \rightarrow \mathbb{R}^{n}$ we set

$$
\nabla_{\Gamma}^{\gamma} \cdot \varphi:=\underline{D}_{m}^{\gamma} \varphi^{m}
$$


to be the anisotropic divergence. Note that we can write

$$
\nabla_{\Gamma}^{\gamma} \cdot \varphi=\tilde{P}^{\gamma}: \nabla \varphi=\operatorname{tr}\left(P^{\gamma} \nabla \varphi\right)=\operatorname{tr}\left(P^{\gamma} \nabla_{\Gamma} \varphi\right)=\operatorname{tr}\left(P^{\gamma} \nabla_{\Gamma}^{\gamma} \varphi\right)=\underline{D}_{j}^{\gamma}\left(I d^{i}\right) \underline{D}_{i}^{\gamma} \varphi^{j} .
$$

Again one can show that the definition given in (4.19) does only depend on the values of $\varphi$ on $\Gamma$. Indeed if $\varphi \equiv 0$ on $\Gamma$, then $\nabla \varphi^{k}=\nabla \varphi^{k} \cdot v v=\nabla \gamma(v) \cdot \nabla \varphi^{k} \frac{v}{\gamma(v)}\left(\right.$ recall (2.3)), thus $(\nabla \varphi)_{i j}=$ $\partial_{j} \varphi^{i}=\nabla \gamma(\nu) \cdot \nabla \varphi^{i} \frac{\nu^{j}}{\gamma(v)}$ and $\nabla_{\Gamma}^{\gamma} \cdot \varphi=0$.

REMARK 4.1 Note that the following identity holds

$$
\left(P^{\gamma} \nabla_{\Gamma}^{\gamma} x^{i}\right)_{m}=\left(P^{\gamma} \nabla_{\Gamma}^{\gamma} x^{m}\right)_{i}
$$

In the isotropic case this amount to

$$
\underline{D}_{m} x^{i}=\underline{D}_{i} x^{m}=P_{i m} .
$$

The latter property, which implies $\nabla_{\Gamma} \cdot \varphi=P: \nabla_{\Gamma} \varphi=\nabla_{\Gamma} I d: \nabla_{\Gamma} \varphi$, is actually used to provide suitable discretizations of the mean curvature flow ( $[8, \S 4.2])$ and of the Willmore flow ( [10]) in the isotropic setting.

LEMma 4.2 Given smooth maps $f, g: \Gamma \rightarrow \mathbb{R}, \varphi: \Gamma \rightarrow \mathbb{R}^{n}$, defined in a neighbourhood of $\Gamma$, we have that

$$
\begin{aligned}
& \nabla_{\Gamma}^{\gamma}(f+g)=\nabla_{\Gamma}^{\gamma} f+\nabla_{\Gamma}^{\gamma} g, \\
& \nabla_{\Gamma}^{\gamma}(f g)=f \nabla_{\Gamma}^{\gamma} g+g \nabla_{\Gamma}^{\gamma} f, \\
& \nabla_{\Gamma}^{\gamma} \cdot(f \varphi)=f \nabla_{\Gamma}^{\gamma} \cdot \varphi+\nabla_{\Gamma}^{\gamma} f \cdot \varphi .
\end{aligned}
$$

The anisotropic Laplace operator for a smooth function $f: \Gamma \rightarrow \mathbb{R}$ is defined by

$$
\triangle_{\Gamma}^{\gamma} f:=\nabla_{\Gamma}^{\gamma} \cdot\left(\gamma^{\prime \prime}\left(\frac{v}{\gamma(v)}\right) \nabla_{\Gamma}^{\gamma} f\right)
$$

Note that the above definitions of anisotropic tangential gradient, divergence and Laplace operator appear also in [2]. At a point $x \in \Gamma$ with Euclidean unit normal $v$ the anisotropic normal vector is given by

$$
v_{\gamma}:=\gamma^{\prime}(v)
$$

The anisotropic scalar curvature is defined by

$$
h_{\gamma}:=\nabla_{\Gamma}^{\gamma} \cdot v_{\gamma} .
$$

Using the homogeneity property (2.4) one infers that

$$
h_{\gamma}=\nabla_{\Gamma} \cdot v_{\gamma}=\operatorname{div}_{\mathbb{R}^{n}} v_{\gamma}
$$

and thus equivalence between (4.28) and (2.8) can be recognized. The formula for integration by parts is given in the following lemma (compare with the isotropic counterpart (4.10)). 
LEMma 4.3 (Integration by parts) Let $f: \Gamma \rightarrow \mathbb{R}$ be a smooth function. Then we have

$$
\int_{\Gamma} \underline{D}_{m}^{\gamma} f d \mu=\int_{\Gamma} f h_{\gamma} \frac{v_{m}}{\gamma(\nu)} d \mu
$$

where $d \mu$ is the anisotropic area element $d \mu=\gamma(v) d V$.

Proof. Using the definition for the anisotropic gradient, (2.3), and the standard integration by parts formula (4.10) we infer that

$$
\begin{aligned}
\int_{\Gamma} \underline{D}_{m}^{\gamma} f d \mu & =\int_{\Gamma}\left(\delta_{m i}-\gamma_{p_{i}}(v) \frac{v^{m}}{\gamma(v)}\right) \underline{D}_{i} f d \mu \\
= & \int_{\Gamma} \underline{D}_{m}(f \gamma(v)) d V-\int_{\Gamma} f \underline{D}_{m}(\gamma(v)) d V-\int_{\Gamma} \underline{D}_{i}\left(\gamma_{p_{i}}(v) f v^{m}\right) d V \\
& \quad+\int_{\Gamma} f \underline{D}_{i}\left(\gamma_{p_{i}}(v) v^{m}\right) d V \\
= & \int_{\Gamma} f h v^{m} \gamma(v) d V-\int_{\Gamma} f \underline{D}_{m}(\gamma(v)) d V-\int_{\Gamma} f h v^{m} \gamma(v) d V \\
& +\int_{\Gamma} f \underline{D}_{i}\left(\gamma_{p_{i}}(v)\right) v^{m} d V+\int_{\Gamma} f \gamma_{p_{i}}(v) \underline{D}_{i} v^{m} d V
\end{aligned}
$$

The claim now follows using the fact that $\underline{D}_{i}\left(\gamma_{p_{i}}(v)\right)=h_{\gamma}$ (see (4.29)) and $\underline{D}_{i} v^{m}=\underline{D}_{m} v^{i}$ (cf. $[8, \S 2.3]$ ).

As a consequence of Lemma 4.3 we obtain the following

LEMmA 4.4 Let $f: \Gamma \rightarrow \mathbb{R}, \varphi: \Gamma \rightarrow \mathbb{R}^{n}$ be smooth maps. Then we have

$$
\int_{\Gamma} f \nabla_{\Gamma}^{\gamma} \cdot \varphi d \mu=-\int_{\Gamma} \varphi \cdot \nabla_{\Gamma}^{\gamma} f d \mu+\int_{\Gamma} f h_{\gamma} \varphi \cdot \frac{v}{\gamma(\nu)} d \mu
$$

Proof. A straight forward computation gives

$$
\int_{\Gamma} f \nabla_{\Gamma}^{\gamma} \cdot \varphi d \mu=\int_{\Gamma} f \underline{D}_{m}^{\gamma} \varphi^{m} d \mu=\int_{\Gamma} \underline{D}_{m}^{\gamma}\left(f \varphi^{m}\right) d \mu-\int_{\Gamma} \varphi^{m} \underline{D}_{m}^{\gamma} f d \mu .
$$

from which the claim follows using Lemma 4.3.

From the above Lemma and (2.4) we immediately infer the following Green's formulas.

LEMmA 4.5 (Green's formulas) Let $f, \varphi: \Gamma \rightarrow \mathbb{R}$ be smooth functions. Then we have

$$
\int_{\Gamma} \nabla_{\Gamma}^{\gamma} f \cdot \nabla_{\Gamma}^{\gamma} \varphi d \mu=-\int_{\Gamma} \varphi \nabla_{\Gamma}^{\gamma} \cdot\left(P^{\gamma} \nabla_{\Gamma}^{\gamma} f\right) d \mu .
$$

Note that from $\nabla_{\Gamma}^{\gamma} f=\tilde{P}^{\gamma} \nabla_{\Gamma}^{\gamma} f$ we immediately infer

$$
\int_{\Gamma} \nabla_{\Gamma}^{\gamma} f \cdot \nabla_{\Gamma}^{\gamma} \varphi d \mu=\int_{\Gamma}\left(P^{\gamma} \nabla_{\Gamma}^{\gamma} f\right) \cdot \nabla_{\Gamma}^{\gamma} \varphi d \mu=\int_{\Gamma} \nabla_{\Gamma}^{\gamma} f \cdot\left(P^{\gamma} \nabla_{\Gamma}^{\gamma} \varphi\right) d \mu .
$$

Let $f, g: \Gamma \rightarrow \mathbb{R}$ be smooth functions, and $\triangle_{\Gamma}^{\gamma}(\cdot)$ be as in (4.26). Then we have

$$
\int_{\Gamma} g \triangle_{\Gamma}^{\gamma} f d \mu=\int_{\Gamma} g \nabla_{\Gamma}^{\gamma} \cdot\left(\gamma^{\prime \prime}\left(\frac{v}{\gamma(v)}\right) \nabla_{\Gamma}^{\gamma} f\right) d \mu=-\int_{\Gamma} \gamma^{\prime \prime}\left(\frac{v}{\gamma(\nu)}\right) \nabla_{\Gamma}^{\gamma} f \cdot \nabla_{\Gamma}^{\gamma} g d \mu .
$$


REMARK 4.6 Unlike the isotropic setting where $\gamma(\xi)=|\xi|$ and (4.9) holds, in the anisotropic setting we expect that in general

$$
-\nabla_{\Gamma}^{\gamma} \cdot\left(P^{\gamma} \nabla_{\Gamma}^{\gamma} I d\right) \neq h_{\gamma} \frac{v}{\gamma(v)}
$$

and also

$$
-\nabla_{\Gamma}^{\gamma} \cdot\left(\gamma^{\prime \prime}\left(\frac{v}{\gamma(v)}\right) \nabla_{\Gamma}^{\gamma} I d\right) \neq h_{\gamma} \frac{v}{\gamma(v)}
$$

Indeed, the left-hand side of the second expression contains third derivatives of the anisotropy map $\gamma$ as opposed to the right-hand side with only second order derivatives. As for the first expression, we compute

$$
\begin{aligned}
h_{\gamma} \frac{v^{i}}{\gamma(v)} & =\underline{D}_{m}^{\gamma}\left(\gamma_{p_{m}}(v)\right) \frac{v^{i}}{\gamma(v)}=\underline{D}_{m}^{\gamma}\left(\gamma_{p_{m}}(v) \frac{v^{i}}{\gamma(v)}\right)-\gamma_{p_{m}}(v) \underline{D}_{m}^{\gamma}\left(\frac{v^{i}}{\gamma(v)}\right)=\underline{D}_{m}^{\gamma}\left(\gamma_{p_{m}}(v) \frac{v^{i}}{\gamma(v)}\right) \\
& =-\underline{D}_{m}^{\gamma}\left(\delta_{m i}-\gamma_{p_{m}}(\nu) \frac{v^{i}}{\gamma(v)}\right)=-\underline{D}_{m}^{\gamma}\left(P_{m i}^{\gamma}\right)=-\underline{D}_{m}^{\gamma}\left(\underline{D}_{i}^{\gamma} x^{m}\right) .
\end{aligned}
$$

Moreover

$$
\left(P^{\gamma} \nabla_{\Gamma}^{\gamma} I d^{i}\right)_{m}=P_{m k}^{\gamma} \underline{D}_{k}^{\gamma} x^{i}=P_{m k}^{\gamma} P_{i k}^{\gamma}=P_{m k}^{\gamma} \tilde{P}_{k i}^{\gamma}=\left(P^{\gamma} \tilde{P}^{\gamma}\right)_{m i} .
$$

In the isotropic case (4.22) holds (from which (4.9) follows), whereas in the anisotropic setting all we have is (4.21).

REMARK 4.7 Note that, with the notation introduced in this section, an anisotropic Willmore surface $\Gamma$, i.e. a critical point for (2.10), satisfies the equation

$$
0=\frac{1}{2} h_{\gamma}^{3}-\triangle_{\Gamma}^{\gamma} h_{\gamma}-h_{\gamma} \operatorname{tr}\left(\left(\gamma^{\prime \prime}(v) D v\right)^{2}\right) \quad \text { on } \Gamma
$$

(see (2.12), [18, Remark 6.7] and recall also (4.26), (4.27), (4.28)).

\subsection{Variations for the anisotropic area functional}

We start by computing the first variation of the anisotropic area functional (2.2). The map $u$ demotes as usual the identity map on a smooth closed compact oriented hypersurface $\Gamma \subset \mathbb{R}^{n}$.

LEMMA 4.8 The first variation for the anisotropic area functional (2.2) at $u=i d_{\Gamma}$ in direction $\varphi \in C^{\infty}\left(\Gamma, \mathbb{R}^{n}\right)$ is given by

$$
\left\langle A_{\gamma}^{\prime}(u), \varphi\right\rangle=\int_{\Gamma} \nabla_{\Gamma}^{\gamma} \cdot \varphi d \mu
$$

where $d \mu$ is the anisotropic area element $d \mu=\gamma(v) d V$.

Proof. To calculate the first variation we first work for simplicity on a single chart $X: U \rightarrow \mathbb{R}^{n}$, where $U \subset \mathbb{R}^{n-1}$ is an open set, and consider variations of the form

$$
X^{\epsilon}=X+\epsilon \phi
$$


for some $\phi \in C_{0}^{\infty}\left(U, \mathbb{R}^{n}\right)$. We set $\Gamma^{\epsilon}=\Gamma$ on $\Gamma \backslash X(U)$ and $\Gamma^{\epsilon}=X^{\epsilon}(U)$ otherwise, and define $\varphi: \Gamma \rightarrow \mathbb{R}^{n}$ by $\varphi \circ X=\phi$ on $X(U)$ and $\varphi=0$ otherwise.

We have that

$$
\begin{aligned}
\left\langle A_{\gamma}^{\prime}(u), \varphi\right\rangle & =\left.\frac{d}{d \epsilon}\right|_{\epsilon=0} \int_{\Gamma^{\epsilon}} \gamma\left(v^{\epsilon}\right) d V^{\epsilon}=\left.\frac{d}{d \epsilon}\right|_{\epsilon=0} \int_{U} \gamma\left(v^{\epsilon}\right) \sqrt{\operatorname{det}\left(g_{i j}^{\epsilon}\right)} d x \\
& =\int_{\Gamma} \gamma(v) \nabla_{\Gamma} \cdot \varphi-\gamma_{p_{k}}(v) \nu^{l} \underline{D}_{k} \varphi^{l} d V=\int_{\Gamma} \nabla_{\Gamma}^{\gamma} \cdot \varphi \gamma(\nu) d V .
\end{aligned}
$$

To deduce the above equation one uses the Jacobi formula for the derivative of the determinant (see for instance $[13, \S 8]$ ) to infer that

$$
\left.\frac{d}{d \epsilon}\right|_{\epsilon=0} \sqrt{\operatorname{det}\left(g_{i j}^{\epsilon}\right)}=\nabla_{\Gamma} \cdot \varphi \sqrt{g}=\nabla_{\Gamma} I d: \nabla_{\Gamma} \varphi \sqrt{g},
$$

where obviously $g_{i j}^{\epsilon}=\partial_{i} X^{\epsilon} \cdot \partial_{j} X^{\epsilon}$. Moreover we observe that

$$
\left.\partial_{\epsilon}\right|_{\epsilon=0} v_{\epsilon}^{k}=-v^{l} \underline{D}_{k} \varphi^{l} .
$$

(Note that throughout this paper the index $\epsilon$ will appear as a lower index whenever the upper index position contains another piece of information, for example the space index). Indeed it follows from the definition of tangential gradient in local coordinates (4.11) that

$$
v^{l} \underline{D}_{k} \varphi^{l}=v^{l}\left(g^{m j} \partial_{j} \phi^{l} \partial_{m} X^{k}\right)=g^{m j}\left(v \cdot \partial_{j} \phi\right) \partial_{m} X^{k} .
$$

On the other hand, since $\nu^{\epsilon} \cdot v^{\epsilon}=1$ for any $\epsilon$, we infer that the vector $\partial_{\epsilon} \nu^{\epsilon}$ lies in the tangential space of $\Gamma^{\epsilon}$ and $\partial_{\epsilon} \nu^{\epsilon}=-g_{\epsilon}^{m n}\left(\nu^{\epsilon} \cdot \partial_{n} \partial_{\epsilon} X^{\epsilon}\right) \partial_{m} X^{\epsilon}$. Next evaluate this expression at $\epsilon=0$.

Using partition of unity arguments one generalizes variations on single charts to arbitrary variations on the surface.

REMARK 4.9 Observe that Lemma 4.8 and Lemma 4.3 provide a proof for (3.6).

LEMMA 4.10 The second variation of the anisotropic area functional at $u=i d_{\Gamma}$ in direction $\psi, \varphi \in C^{\infty}\left(\Gamma, \mathbb{R}^{n}\right)$ is given by

$$
\begin{aligned}
A_{\gamma}^{\prime \prime}(u)(\varphi, \psi)=\int_{\Gamma} \nabla_{\Gamma}^{\gamma} \cdot \psi \nabla_{\Gamma}^{\gamma} \cdot \varphi d \mu & -\int_{\Gamma} \underline{D}_{l}^{\gamma} \varphi^{s} \underline{D}_{s}^{\gamma} \psi^{l} d \mu \\
& +\int_{\Gamma} \frac{\nu^{s}}{\gamma(v)} \frac{\nu^{l}}{\gamma(v)} \gamma_{p_{k} p_{m}}\left(\frac{v}{\gamma(v)}\right) \underline{D}_{m}^{\gamma} \varphi^{s} \underline{D}_{k}^{\gamma} \psi^{l} d \mu,
\end{aligned}
$$

where $d \mu$ is the anisotropic area element $d \mu=\gamma(\nu) d V$.

Proof. To compute the second variation we consider again for simplicity variations on a single chart as in (4.34). The general case can be obtained by using partition of unity arguments. Given a map $\psi \in C^{\infty}\left(\Gamma, \mathbb{R}^{n}\right)$, we define a family of smooth maps on $\Gamma^{\epsilon}$ by setting $\tilde{\psi}: \Gamma^{\epsilon} \rightarrow \mathbb{R}^{n}$, $\tilde{\psi} \circ X^{\epsilon}=\psi \circ X$ on $X^{\epsilon}(U)$ and $\tilde{\psi}=\psi$ on $\Gamma \backslash X(U)$ (recall that $\Gamma^{\epsilon}=\Gamma$ on $\Gamma \backslash X(U)$ ). Furthermore let $\Psi: U \rightarrow \mathbb{R}^{n}$ be defined by $\Psi=\psi \circ X$. The second variation in directions $\psi$ and 
$\varphi$ is given by

$$
\begin{aligned}
A_{\gamma}^{\prime \prime}(u)(\varphi, \psi) & =\left.\frac{d}{d \epsilon}\right|_{\epsilon=0} \int_{\Gamma^{\epsilon}} \nabla_{\Gamma^{\epsilon}}^{\gamma} \cdot \tilde{\psi} \gamma\left(v^{\epsilon}\right) d V^{\epsilon} \\
= & \left.\frac{d}{d \epsilon}\right|_{\epsilon=0}\left\{\int_{U}\left(\nabla_{\Gamma^{\epsilon}} I d: \nabla_{\Gamma^{\epsilon}} \tilde{\psi}-\gamma_{p_{k}}\left(v^{\epsilon}\right) \frac{v_{\epsilon}^{l}}{\gamma\left(v^{\epsilon}\right)} \underline{D}_{k}^{\epsilon} \tilde{\psi}^{l}\right) \sqrt{\operatorname{det}\left(g_{i j}^{\epsilon}\right)} \gamma\left(v^{\epsilon}\right) d x\right. \\
& \left.+\int_{\Gamma \backslash X(U)} \nabla_{\Gamma}^{\gamma} \cdot \psi d \mu\right\}
\end{aligned}
$$

Next we look at the differentiation of the single terms in the first integral. From the calculations performed to evaluate the first variation for the anisotropic area functional we already know that

$$
\left.\frac{d}{d \epsilon}\right|_{\epsilon=0} \sqrt{\operatorname{det}\left(g_{i j}^{\epsilon}\right)} \gamma\left(v^{\epsilon}\right)=\nabla_{\Gamma}^{\gamma} \cdot \varphi \gamma(v) \sqrt{g} .
$$

Next we infer that

$$
\left.\frac{d}{d \epsilon}\right|_{\epsilon=0} \frac{v_{\epsilon}^{k}}{\gamma\left(v^{\epsilon}\right)}=-\frac{v^{l}}{\gamma(v)} \underline{D}_{k}^{\gamma} \varphi^{l}
$$

Indeed, using (4.37) and (2.3) we get

$$
\begin{aligned}
\left.\frac{d}{d \epsilon}\right|_{\epsilon=0} \frac{v_{\epsilon}^{k}}{\gamma\left(v^{\epsilon}\right)} & =-\frac{v^{l}}{\gamma(v)} \underline{D}_{k} \varphi^{l}+\frac{v^{k}}{\gamma(v)^{2}} \gamma_{p_{m}}(v) v^{s} \underline{D}_{m} \varphi^{s}=-\frac{v^{l}}{\gamma(v)} \partial_{k} \varphi^{l}+\frac{v^{k} v^{s}}{\gamma(v)^{2}} \nabla \gamma(v) \cdot \nabla \varphi^{s} \\
& =-\frac{v^{l}}{\gamma(v)}\left(\underline{D}_{k}^{\gamma} \varphi^{l}+\frac{v_{k}}{\gamma(v)} \nabla \gamma(v) \cdot \nabla \varphi^{l}\right)+\frac{\nu^{k} v^{s}}{\gamma(v)^{2}} \nabla \gamma(\nu) \cdot \nabla \varphi^{s}=-\frac{v^{l}}{\gamma(v)} \underline{D}_{k}^{\gamma} \varphi^{l} .
\end{aligned}
$$

Next we show that

$$
\left.\frac{d}{d \epsilon}\right|_{\epsilon=0}\left(\nabla_{\Gamma^{\epsilon}} I d: \nabla_{\Gamma^{\epsilon}} \tilde{\psi}\right)=\nabla_{\Gamma} \varphi: \nabla_{\Gamma} \psi-\left(D(\varphi) \nabla_{\Gamma} \psi^{l}\right) \cdot \nabla_{\Gamma} I d^{l},
$$

where $D(\varphi)$ is the symmetric matrix with entries

$$
D(\varphi)_{i j}=\underline{D}_{i} \varphi^{j}+\underline{D}_{j} \varphi^{i}
$$

Using (4.12) we get that

$$
\nabla_{\Gamma^{\epsilon}} I d: \nabla_{\Gamma^{\epsilon}} \tilde{\psi}=g_{\epsilon}^{i j} \partial_{j} X^{\epsilon} \cdot \partial_{i} \Psi
$$

Using the fact that from $g_{\epsilon}^{i j}=g_{\epsilon}^{i k} g_{\epsilon}^{j l} g_{k l}^{\epsilon}$ we can infer that

$$
\left.\frac{d}{d \epsilon}\right|_{\epsilon=0} g_{\epsilon}^{i j}=-\left.g^{i k} g^{j l} \frac{d}{d \epsilon}\right|_{\epsilon=0} g_{k l}^{\epsilon}=-g^{i k} g^{j l}\left(\partial_{k} \phi \cdot \partial_{l} X+\partial_{l} \phi \cdot \partial_{k} X\right),
$$

we derive that

$$
\left.\frac{d}{d \epsilon}\right|_{\epsilon=0}\left(\nabla_{\Gamma^{\epsilon}} I d: \nabla_{\Gamma} \epsilon \tilde{\psi}\right)=-g^{i k} g^{j l} \partial_{j} X \cdot \partial_{i} \Psi\left(\partial_{l} X \cdot \partial_{k} \phi+\partial_{l} \phi \cdot \partial_{k} X\right)+\nabla_{\Gamma} \varphi: \nabla_{\Gamma} \psi .
$$


On the other hand, using (4.11), we have that

$$
\begin{aligned}
\left(D(\varphi) \nabla_{\Gamma} \psi^{l}\right) \cdot \nabla_{\Gamma} I d^{q} & =\left(\underline{D}_{j} \varphi^{i}+\underline{D}_{i} \varphi^{j}\right) \underline{D}_{i} \psi^{l} \underline{D}_{j} I d^{q} \\
& =\left(g^{m k} \partial_{k} \phi^{i} \partial_{m} X^{j}+g^{m k} \partial_{k} \phi^{j} \partial_{m} X^{i}\right) g^{n p} \partial_{p} \Psi^{l} \partial_{n} X^{i} g^{r s} \partial_{s} X^{q} \partial_{r} X^{j} \\
& =g^{n p} g^{k s}\left(\partial_{k} \phi \cdot \partial_{n} X\right)\left(\partial_{p} \Psi^{l} \partial_{s} X^{q}\right)+g^{n p} g^{r s}\left(\partial_{n} \phi \cdot \partial_{r} X\right)\left(\partial_{p} \Psi^{l} \partial_{s} X^{q}\right) \\
& =g^{n p} g^{k s}\left(\partial_{p} \Psi^{l} \partial_{s} X^{q}\right)\left(\partial_{k} \phi \cdot \partial_{n} X+\partial_{n} \phi \cdot \partial_{k} X\right) .
\end{aligned}
$$

If $q=l$ this gives

$$
\begin{aligned}
\left(D(\varphi) \nabla_{\Gamma} \psi^{l}\right) \cdot \nabla_{\Gamma} I d^{l} & =g^{n p} g^{k s}\left(\partial_{p} \Psi \cdot \partial_{s} X\right)\left(\partial_{k} \phi \cdot \partial_{n} X+\partial_{n} \phi \cdot \partial_{k} X\right) \\
& =g^{i k} g^{j l}\left(\partial_{i} \Psi \cdot \partial_{j} X\right)\left(\partial_{l} \phi \cdot \partial_{k} X+\partial_{k} \phi \cdot \partial_{l} X\right)
\end{aligned}
$$

where in the last step we have renamed the indices as follows: $k \rightarrow l, p \rightarrow i, s \rightarrow j, n \rightarrow k$. Therefore we have shown that (4.42) holds.

Next we show that

$$
\left.\partial_{\epsilon}\right|_{\epsilon=0} \underline{D}_{k}^{\epsilon} \tilde{\psi}^{l}=\nabla_{\Gamma} \psi^{l} \cdot \nabla_{\Gamma} \varphi^{k}-\left(D(\varphi) \nabla_{\Gamma} \psi^{l}\right) \cdot \nabla_{\Gamma} I d^{k}
$$

Indeed, using (4.44) and the fact that $\underline{D}_{k}^{\epsilon} \tilde{\psi}^{l}=g_{\epsilon}^{m j} \partial_{j} \Psi^{l} \partial_{m} X_{\epsilon}^{k}$, we obtain

$$
\left.\partial_{\epsilon}\right|_{\epsilon=0} \underline{D}_{k}^{\epsilon} \tilde{\psi}^{l}=\left\{-g^{m r} g^{j s}\left(\partial_{r} \phi \cdot \partial_{s} X+\partial_{s} \phi \cdot \partial_{r} X\right) \partial_{j} \Psi^{l} \partial_{m} X^{k}+g^{m j} \partial_{j} \Psi^{l} \partial_{m} \phi^{k}\right\} .
$$

Equation (4.46) now follows from (4.12) and (4.45) (after renaming indices in (4.45) as follows: $k \rightarrow r, n \rightarrow s, p \rightarrow j, s \rightarrow m)$.

Using (4.42), (4.41), (4.46), (2.3), and (2.4), we can write

$$
\begin{aligned}
\left.\frac{d}{d \epsilon}\right|_{\epsilon=0} & \nabla_{\Gamma^{\epsilon}}^{\gamma} \cdot \tilde{\psi} \\
= & \left.\frac{d}{d \epsilon}\right|_{\epsilon=0}\left(\nabla_{\Gamma^{\epsilon}} I d: \nabla_{\Gamma^{\epsilon}} \tilde{\psi}-\gamma_{p_{k}}\left(\frac{v^{\epsilon}}{\gamma\left(v^{\epsilon}\right)}\right) \frac{v_{\epsilon}^{l}}{\gamma\left(\nu^{\epsilon}\right)} \underline{D}_{k}^{\epsilon} \tilde{\psi}^{l}\right) \\
= & \nabla_{\Gamma} \varphi: \nabla_{\Gamma} \psi-\left(D(\varphi) \nabla_{\Gamma} \psi^{l}\right) \cdot \nabla_{\Gamma} I d^{l}+\gamma_{p_{k} p_{m}}\left(\frac{v}{\gamma(v)}\right) \frac{v^{s}}{\gamma(v)} \underline{D}_{m}^{\gamma} \varphi^{s} \frac{v^{l}}{\gamma(v)} \underline{D}_{k} \psi^{l} \\
& +\gamma_{p_{k}}(v) \frac{v^{s}}{\gamma(v)} \underline{D}_{l}^{\gamma} \varphi^{s} \underline{D}_{k} \psi^{l}-\gamma_{p_{k}}(v) \frac{v^{l}}{\gamma(v)}\left(\nabla_{\Gamma} \psi^{l} \cdot \nabla_{\Gamma} \varphi^{k}-\left(D(\varphi) \nabla_{\Gamma} \psi^{l}\right) \cdot \nabla_{\Gamma} I d^{k}\right) \\
= & \gamma_{p_{k} p_{m}}\left(\frac{v}{\gamma(v)}\right) \frac{v^{s}}{\gamma(v)} \underline{D}_{m}^{\gamma} \varphi^{s} \frac{v^{l}}{\gamma(v)} \underline{D}_{k}^{\gamma} \psi^{l}+Q,
\end{aligned}
$$

where

$$
\begin{aligned}
Q:= & \nabla_{\Gamma} \varphi: \nabla_{\Gamma} \psi-\left(D(\varphi) \nabla_{\Gamma} \psi^{l}\right) \cdot \nabla_{\Gamma} I d^{l} \\
& +\gamma_{p_{k}}(v) \frac{v^{s}}{\gamma(v)} \underline{D}_{l}^{\gamma} \varphi^{s} \underline{D}_{k} \psi^{l}-\gamma_{p_{k}}(v) \frac{v^{l}}{\gamma(v)}\left(\nabla_{\Gamma} \psi^{l} \cdot \nabla_{\Gamma} \varphi^{k}-\left(D(\varphi) \nabla_{\Gamma} \psi^{l}\right) \cdot \nabla_{\Gamma} I d^{k}\right) .
\end{aligned}
$$


Next we show that

$$
Q=-\underline{D}_{l}^{\gamma} \varphi^{s} \underline{D}_{s}^{\gamma} \psi^{l}
$$

We have that

$$
\begin{aligned}
& Q=\underline{D}_{i} \varphi^{j} \underline{D}_{i} \psi^{j}-\left(D(\varphi) \nabla_{\Gamma} \psi^{l}\right) \cdot \nabla_{\Gamma} I d^{l}+\gamma_{p_{k}}(v) \frac{\nu^{s}}{\gamma(v)} \underline{D}_{l}^{\gamma} \varphi^{s}\left(\partial_{k} \psi^{l}-\nabla \psi \psi^{l} \cdot v v^{k}\right) \\
& -\gamma_{p_{k}}(v) \frac{\nu^{l}}{\gamma(v)}\left(\underline{D}_{i} \psi^{l} \cdot \underline{D}_{i} \varphi^{k}-\left(D(\varphi) \nabla_{\Gamma} \psi^{l}\right) \cdot \nabla_{\Gamma} I d^{k}\right) \\
& =P_{k l}^{\gamma} \underline{D}_{i} \psi^{l} \cdot \underline{D}_{i} \varphi^{k}-P_{k l}^{\gamma}\left(\underline{D}_{m} \varphi^{s}+\underline{D}_{s} \varphi^{m}\right) \underline{D}_{s} \psi^{l} \underline{D}_{m} I d^{k}+\gamma_{p_{k}}(v) \frac{\nu^{s}}{\gamma(v)} \underline{D}_{l}^{\gamma} \varphi^{s} \underline{D}_{k}^{\gamma} \psi^{l} \\
& +\frac{v^{s}}{\gamma(v)} \underline{D}_{l}^{\gamma} \varphi^{s} \nabla \gamma(v) \cdot \nabla \psi^{l}-v^{s} \underline{D}_{l}^{\gamma} \varphi^{s} \nabla \psi^{l} \cdot v \\
& =P_{k l}^{\gamma} P_{i m} \partial_{m} \psi^{l} P_{i s} \partial_{s} \varphi^{k}-P_{k l}^{\gamma}\left(P_{m i} \partial_{i} \varphi^{s}+P_{s q} \partial_{q} \varphi^{m}\right) P_{s n} \partial_{n} \psi^{l} P_{k m} \\
& +\gamma_{p_{k}}(v) \frac{\nu^{s}}{\gamma(v)} \underline{D}_{l}^{\gamma} \varphi^{s} \underline{D}_{k}^{\gamma} \psi^{l} \\
& -\underline{D}_{l}^{\gamma} \varphi^{s} \underline{D}_{s}^{\gamma} \psi^{l}+\underline{D}_{l}^{\gamma} \varphi^{s} \partial_{s} \psi^{l}-v^{s} \underline{D}_{l}^{\gamma} \varphi^{s} \nabla \psi^{l} \cdot v \\
& =-\underline{D}_{l}^{\gamma} \varphi^{s} \underline{D}_{s}^{\gamma} \psi^{l}+P_{k l}^{\gamma} P_{m s} \partial_{m} \psi^{l} \partial_{s} \varphi^{k}-P_{k l}^{\gamma} P_{k i} P_{s n} \partial_{i} \varphi^{s} \partial_{n} \psi^{l}-P_{k l}^{\gamma} P_{q n} P_{k m} \partial_{q} \varphi^{m} \partial_{n} \psi^{l} \\
& +\underline{D}_{l}^{\gamma} \varphi^{s} \underline{D}_{s} \psi^{l} \quad \text { since } \gamma_{p_{k}}(v) \underline{D}_{k}^{\gamma} \psi^{l}=0 \\
& =-\underline{D}_{l}^{\gamma} \varphi^{s} \underline{D}_{s}^{\gamma} \psi^{l}+P_{k l}^{\gamma} P_{m s} \partial_{m} \psi^{l} \partial_{s} \varphi^{k}-P_{i l}^{\gamma} P_{s n} \partial_{i} \varphi^{s} \partial_{n} \psi^{l}-P_{m l}^{\gamma} P_{q n} \partial_{q} \varphi^{m} \partial_{n} \psi^{l} \\
& +P_{k l}^{\gamma} \partial_{k} \varphi^{s} P_{s n} \partial_{n} \psi^{l} \quad \text { due to (4.16) } \\
& =-\underline{D}_{l}^{\gamma} \varphi^{s} \underline{D}_{s}^{\gamma} \psi^{l}
\end{aligned}
$$

Using (4.39), (4.40), (4.47) and (4.48) we can finally state that

$$
\begin{aligned}
A_{\gamma}^{\prime \prime}(u)(\varphi, \psi)=\int_{\Gamma} \nabla_{\Gamma}^{\gamma} \cdot \psi \nabla_{\Gamma}^{\gamma} \cdot \varphi d \mu-\int_{\Gamma} & \underline{D}_{l}^{\gamma} \varphi^{s} \underline{D}_{s}^{\gamma} \psi^{l} d \mu \\
& \quad+\int_{\Gamma} \frac{v^{s}}{\gamma(v)} \frac{v^{l}}{\gamma(v)} \gamma_{p_{k} p_{m}}\left(\frac{v}{\gamma(v)}\right) \underline{D}_{m}^{\gamma} \varphi^{s} \underline{D}_{k}^{\gamma} \psi^{l} d \mu
\end{aligned}
$$

Note that in the isotropic case where $\gamma(v)=|\nu|$ we recover the results presented in [10, Lemma 1 and Lemma 2].

\subsection{First variation for the anisotropic Willmore functional}

Next we derive the first variation for the anisotropic Willmore functional (2.10)

$$
W_{\gamma}(u)=\frac{1}{2} \int_{\Gamma} h_{\gamma}^{2} \gamma(\nu) d V=\frac{1}{2} \int_{\Gamma} \gamma^{*}\left(\kappa_{\gamma}\right)^{2} d \mu=\frac{1}{2} \int_{\Gamma} \gamma\left(T\left(\kappa_{\gamma}\right)\right)^{2} d \mu
$$


at $u=i d_{\Gamma}$. For the computation we use the weak formulation for the anisotropic mean curvature vector $\kappa_{\gamma}=-h_{\gamma} v_{\gamma}$ (recall (2.9), (4.27), (4.29)) given in Problem 3.3 and the result derived in Lemma 4.8.

Observe that if $\kappa_{\gamma} \in L^{2}(\Gamma)^{n}$, then so does $T\left(\kappa_{\gamma}\right)=-h_{\gamma} \frac{v}{\gamma(v)}$, due to $\gamma\left(T\left(\kappa_{\gamma}\right)\right)=\gamma^{*}\left(\kappa_{\gamma}\right)$ and the equivalence of norms in $\mathbb{R}^{n}$. The reverse statement is also true.

LEMMA 4.11 Let $\kappa_{\gamma}$ satisfy

$$
\int_{\Gamma} T\left(\kappa_{\gamma}\right) \cdot \psi d \mu+\int_{\Gamma} \nabla_{\Gamma}^{\gamma} \cdot \psi d \mu=0 \quad \forall \psi \in C^{\infty}(\Gamma) .
$$

The first variation of the anisotropic Willmore functional at $u=i d_{\Gamma}$ in direction $\varphi \in C^{\infty}\left(\Gamma, \mathbb{R}^{n}\right)$ is given by

$$
\begin{aligned}
\left\langle W_{\gamma}^{\prime}(u), \varphi\right\rangle=- & \frac{1}{2} \int_{\Gamma} \gamma\left(T\left(\kappa_{\gamma}\right)\right)^{2} \nabla_{\Gamma}^{\gamma} \cdot \varphi d \mu-\int_{\Gamma} \nabla_{\Gamma}^{\gamma} \cdot \kappa_{\gamma} \nabla_{\Gamma}^{\gamma} \cdot \varphi d \mu \\
& +\int_{\Gamma} \underline{D}_{l}^{\gamma} \varphi^{s} \underline{D}_{s}^{\gamma} \kappa_{\gamma}^{l} d \mu-\int_{\Gamma} \frac{\nu^{s}}{\gamma(v)} \frac{v^{l}}{\gamma(v)} \gamma_{p_{k} p_{m}}\left(\frac{v}{\gamma(v)}\right) \underline{D}_{m}^{\gamma} \varphi^{s} \underline{D}_{k}^{\gamma} \kappa_{\gamma}^{l} d \mu,
\end{aligned}
$$

where $d \mu$ is the anisotropic area element $d \mu=\gamma(v) d V$.

Proof. As for the proof of the first variation of the area functional, we first work on a single chart $X: U \rightarrow \mathbb{R}^{n}$, where $U \subset \mathbb{R}^{n-1}$ is an open set, and consider variations of the form $X^{\epsilon}=X+\epsilon \phi$ for some $\phi \in C_{0}^{\infty}\left(U, \mathbb{R}^{n}\right)$. We set $\Gamma^{\epsilon}=\Gamma$ on $\Gamma \backslash X(U)$ and $\Gamma^{\epsilon}=X^{\epsilon}(U)$ otherwise, and define $\varphi: \Gamma \rightarrow \mathbb{R}^{n}$ by $\varphi \circ X=\phi$ on $X(U)$ and $\varphi=0$ otherwise. Using the results from Lemma 4.8 and the definition and properties of the duality map $T^{-1}=\frac{1}{2} \partial\left(\gamma^{2}\right)$ (cf. also [21, Proposition 47.19] or comments in Section 2) we infer that

$$
\begin{aligned}
\left\langle W_{\gamma}^{\prime}(u), \varphi\right\rangle & =\left.\frac{d}{d \epsilon}\right|_{\epsilon=0} \frac{1}{2} \int_{\Gamma^{\epsilon}} \gamma\left(T\left(\kappa_{\gamma}^{\epsilon}\right)\right)^{2} \gamma\left(\nu^{\epsilon}\right) d V^{\epsilon}=\left.\frac{d}{d \epsilon}\right|_{\epsilon=0} \frac{1}{2} \int_{U} \gamma\left(T\left(\kappa_{\gamma}^{\epsilon}\right)\right)^{2} \gamma\left(v^{\epsilon}\right) \sqrt{\operatorname{det}\left(g_{i j}^{\epsilon}\right)} d x \\
& =\left.\int_{U} T^{-1}\left(T\left(\kappa_{\gamma}\right)\right) \cdot \frac{d}{d \epsilon}\right|_{\epsilon=0} T\left(\kappa_{\gamma}^{\epsilon}\right) \gamma(\nu) \sqrt{\operatorname{det}\left(g_{i j}\right)} d x+\frac{1}{2} \int_{\Gamma} h_{\gamma}^{2} \nabla_{\Gamma}^{\gamma} \cdot \varphi d \mu \\
& =\left.\int_{\Gamma} \frac{d}{d \epsilon}\right|_{\epsilon=0} T\left(\kappa_{\gamma}^{\epsilon}\right) \cdot \kappa_{\gamma} d \mu+\frac{1}{2} \int_{\Gamma} h_{\gamma}^{2} \nabla_{\Gamma}^{\gamma} \cdot \varphi d \mu .
\end{aligned}
$$

Note that under our assumptions $T\left(\kappa_{\gamma}^{\epsilon}\right)=-h_{\gamma}^{\epsilon} \frac{v^{\epsilon}}{\gamma\left(v_{\epsilon}\right)}$ (recall also (4.29)) is smooth enough for its derivative with respect to $\epsilon$ to exist. However it is important to point out that when taking the partial derivative with respect to $\epsilon$ we can not apply the chain rule, since $T$ is not necessarily Frechet differentiable in zero (regardless how smooth $\gamma^{*}$ might be. Indeed, $T$ has at the origin directional derivative in direction $\xi \in \mathbb{R}^{n} \backslash\{0\}$ equal to $\lim _{h \rightarrow 0} \frac{T(0+h \xi)-T(0)}{h}=T(\xi)=\gamma^{*}(\xi)\left(\gamma^{*}\right)^{\prime}(\xi)$, which is not necessarily linear with respect to $\xi$ ).

To determine the first integral in (4.51) we use the weak formulation for the anisotropic mean curvature vector (4.49) and Lemma 4.10. Given a map $\psi \in C^{\infty}\left(\Gamma, \mathbb{R}^{n}\right)$, we define a family of smooth maps on $\Gamma^{\epsilon}$ by setting $\tilde{\psi}: \Gamma^{\epsilon} \rightarrow \mathbb{R}^{n}, \tilde{\psi} \circ X^{\epsilon}=\psi \circ X$ on $X^{\epsilon}(U)$ and $\tilde{\psi}=\psi$ on $\Gamma \backslash X(U)$ (recall that $\Gamma^{\epsilon}=\Gamma$ on $\Gamma \backslash X(U)$ ). By differentiating with respect to $\epsilon$ the equation

$$
\int_{\Gamma^{\epsilon}} T\left(\kappa_{\gamma}^{\epsilon}\right) \cdot \tilde{\psi} d \mu^{\epsilon}+\int_{\Gamma^{\epsilon}} \nabla_{\Gamma^{\epsilon}}^{\gamma} \cdot \tilde{\psi} d \mu^{\epsilon}=0
$$


we obtain

$$
\left.\int_{\Gamma} \frac{d}{d \epsilon}\right|_{\epsilon=0} T\left(\kappa_{\gamma}^{\epsilon}\right) \cdot \psi d \mu+\int_{\Gamma} T\left(\kappa_{\gamma}\right) \cdot \psi \nabla_{\Gamma}^{\gamma} \cdot \varphi d \mu+A_{\gamma}^{\prime \prime}(u)(\varphi, \psi)=0 \quad \forall \psi \in C^{\infty}\left(\Gamma, \mathbb{R}^{n}\right) .
$$

By an approximation argument (if necessary) we can take $\psi=\kappa_{\gamma}$ in the above equation; together with (4.51) we obtain

$$
\left\langle W_{\gamma}^{\prime}(u), \varphi\right\rangle=\frac{1}{2} \int_{\Gamma} h_{\gamma}^{2} \nabla_{\Gamma}^{\gamma} \cdot \varphi d \mu-\int_{\Gamma} T\left(\kappa_{\gamma}\right) \cdot \kappa_{\gamma} \nabla_{\Gamma}^{\gamma} \cdot \varphi d \mu-A_{\gamma}^{\prime \prime}(u)\left(\varphi, \kappa_{\gamma}\right)
$$

and the claim now follows from Lemma 4.10 and the fact that $T\left(\kappa_{\gamma}\right) \cdot \kappa_{\gamma}=\gamma\left(T\left(\kappa_{\gamma}\right)\right)^{2}=\gamma^{*}\left(\kappa_{\gamma}\right)^{2}=$ $h_{\gamma}^{2}$.

The general case where the variation is not contained in a single chart follows by using partition of unity.

In Section 3.1 a flow geometrically equivalent to (3.2) (see also Definition 3.1) was discussed. Since, as observed in Section 3.1, the Willmore functional can be written as

$$
W_{\gamma}(u)=\frac{1}{2} \int_{\Gamma} h_{\gamma}^{2} \gamma(v) d V=\frac{1}{2} \int_{\Gamma}\left|\frac{\kappa_{\gamma}^{N}}{\gamma(v)}\right|^{2} \gamma(v) d V,
$$

where $\kappa_{\gamma}^{N}=\kappa_{\gamma} \cdot v v=-\gamma(v) h_{\gamma} v$, we infer from (3.10), Lemma 4.8, and using the same arguments and notation of the proof of Lemma 4.11 that

$$
\int_{\Gamma^{\epsilon}} \frac{\left(\kappa_{\gamma}^{N}\right)^{\epsilon}}{\gamma\left(v_{\epsilon}\right)} \cdot \tilde{\psi} d V^{\epsilon}+\int_{\Gamma^{\epsilon}} \nabla_{\Gamma^{\epsilon}}^{\gamma} \cdot \tilde{\psi} d \mu^{\epsilon}=0 \quad \forall \psi \in C^{\infty}\left(\Gamma, \mathbb{R}^{n}\right) .
$$

and (together with (4.36) and (4.39))

$$
\left.\int_{\Gamma} \frac{d}{d \epsilon}\right|_{\epsilon=0} \frac{\left(\kappa_{\gamma}^{N}\right)^{\epsilon}}{\gamma\left(v^{\epsilon}\right)} \cdot \psi d V+\int_{\Gamma} \frac{\kappa_{\gamma}^{N}}{\gamma(v)} \cdot \psi \nabla_{\Gamma} \cdot \varphi d V+A_{\gamma}^{\prime \prime}(u)(\varphi, \psi)=0 \quad \forall \psi \in C^{\infty}\left(\Gamma, \mathbb{R}^{n}\right),
$$

so that

$$
\left\langle W_{\gamma}^{\prime}(u), \varphi\right\rangle=\frac{1}{2} \int_{\Gamma}\left|\frac{\kappa_{\gamma}^{N}}{\gamma(v)}\right|^{2} \nabla_{\Gamma}^{\gamma} \cdot \varphi d \mu-\int_{\Gamma}\left|\frac{\kappa_{\gamma}^{N}}{\gamma(v)}\right|^{2} \nabla_{\Gamma} \cdot \varphi d \mu-A_{\gamma}^{\prime \prime}(u)\left(\varphi, \kappa_{\gamma}^{N}\right) .
$$

Therefore we obtain

LEMMA 4.12 Let $\kappa_{\gamma}^{N}$ satisfy

$$
\int_{\Gamma} \frac{\kappa_{\gamma}^{N}}{\gamma(\nu)} \cdot \psi d V+\int_{\Gamma} \nabla_{\Gamma}^{\gamma} \cdot \psi d \mu=0 \quad \forall \psi \in C^{\infty}(\Gamma) .
$$

The first variation of the anisotropic Willmore functional at $u=i d_{\Gamma}$ in direction $\varphi \in C^{\infty}\left(\Gamma, \mathbb{R}^{n}\right)$ is given by

$$
\begin{aligned}
\left\langle W_{\gamma}^{\prime}(u), \varphi\right\rangle= & \frac{1}{2} \int_{\Gamma}\left|\frac{\kappa_{\gamma}^{N}}{\gamma(v)}\right|^{2} \nabla_{\Gamma}^{\gamma} \cdot \varphi d \mu-\int_{\Gamma}\left|\frac{\kappa_{\gamma}^{N}}{\gamma(v)}\right|^{2} \nabla_{\Gamma} \cdot \varphi d \mu-\int_{\Gamma} \nabla_{\Gamma}^{\gamma} \cdot \kappa_{\gamma}^{N} \nabla_{\Gamma}^{\gamma} \cdot \varphi d \mu \quad(4.54 \\
& +\int_{\Gamma} \underline{D}_{l}^{\gamma} \varphi^{s} \underline{D}_{S}^{\gamma}\left(\kappa_{\gamma}^{N}\right)^{l} d \mu-\int_{\Gamma} \frac{\nu^{s}}{\gamma(v)} \frac{\nu^{l}}{\gamma(\nu)} \gamma_{p_{k}} p_{m}\left(\frac{v}{\gamma(v)}\right) \underline{D}_{m}^{\gamma} \varphi^{s} \underline{D}_{k}^{\gamma}\left(\kappa_{\gamma}^{N}\right)^{l} d \mu,
\end{aligned}
$$


where $d \mu$ is the anisotropic area element $d \mu=\gamma(v) d V$.

\subsection{A formulation of the flow that is suitable for discretization}

As in [10] we assume that the evolving surfaces $\Gamma(t)$ are smooth in space and time and that all the quantities that we shall use make sense. Let $G_{\bar{T}}$ be the topological cylinder

$$
G_{\bar{T}}=\bigcup_{t \in[0, \bar{T}]} \Gamma(t) \times\{t\} .
$$

We assume that for each $t \in[0, \bar{T}]$ the surface $\Gamma(t)$ is regularly and smoothly parametrised locally by $X=X(x, t), x \in U \subset \mathbb{R}^{n-1}$. Denote by $u: G_{\bar{T}} \rightarrow \mathbb{R}^{n}$ the map

$$
u(X(x, t), t)=X(x, t), \quad x \in U, \quad t \in[0, \bar{T}] .
$$

For a function $\eta: G_{\bar{T}} \rightarrow \mathbb{R}$, the quantity $\dot{\eta}$ denotes its material derivative

$$
\dot{\eta}=\eta_{t}+v \cdot \nabla \eta,
$$

where $V$ is the velocity of the surface $\Gamma(t)$ given by $V(X(x, t), t)=X_{t}(x, t)$ and $\nabla \eta$ is the usual $n$-dimensional spatial gradient of a smooth extension of $\eta$ to a neighborhood of $G_{\bar{T}}$. In particular $\dot{\eta}(X(\cdot, t), t)$ is equal to the time derivative of $\eta(X(\cdot, t), t)$. Note that the material derivative of the identity map $u$ is the velocity, i.e.

$$
\dot{u}(X(x, t), t)=X_{t}(x, t) \in \mathbb{R}^{n} .
$$

Following this notation the evolution problem formulated in Definition 3.1 reads as follows.

PROBLEM 4.13 (Anisotropic Willmore flow) For a given hypersurface $\Gamma_{0} \subset \mathbb{R}^{n}$ determine a family of smooth hypersurfaces $(\Gamma(t))_{t \in[0, \bar{T}]}$ with $\Gamma(0)=\Gamma_{0}$, which move according to the law

$$
\int_{\Gamma} T(\dot{u}) \cdot \varphi d \mu=-\left\langle W_{\gamma}^{\prime}(u), \varphi\right\rangle
$$

for every test function $\varphi$ and on the time interval $(0, \bar{T}]$.

By Lemma 4.11 a mixed formulation for the anisotropic Willmore flow (recall also Problem 3.3), which allows for a discretization by piecewise linear finite elements, is the following.

PROBLEM 4.14 For given initial surface $\Gamma_{0}$ and $u_{0}=i d_{\Gamma_{0}}$ determine $u, \kappa_{\gamma}: G_{\bar{T}} \rightarrow \mathbb{R}^{n}$ such that $u=i d_{\Gamma}, u(\cdot, 0)=u_{0}$ on $\Gamma_{0}$, and

$$
\begin{aligned}
\int_{\Gamma} T(\dot{u}) \cdot \varphi d \mu-\frac{1}{2} \int_{\Gamma} \gamma\left(T\left(\kappa_{\gamma}\right)\right)^{2} \nabla_{\Gamma}^{\gamma} \cdot \varphi d \mu-\int_{\Gamma} \nabla_{\Gamma}^{\gamma} \cdot \kappa_{\gamma} \nabla_{\Gamma}^{\gamma} \cdot \varphi d \mu \\
+\int_{\Gamma} \underline{D}_{l}^{\gamma} \varphi^{s} \underline{D}_{s}^{\gamma} \kappa_{\gamma}^{l} d \mu-\int_{\Gamma} \frac{\nu^{s}}{\gamma(v)} \frac{\nu^{l}}{\gamma(v)} \gamma_{p_{k}} p_{m}\left(\frac{v}{\gamma(v)}\right) \underline{D}_{m}^{\gamma} \varphi^{s} \underline{D}_{k}^{\gamma} \kappa_{\gamma}^{l} d \mu=0, \\
\quad \int_{\Gamma} T\left(\kappa_{\gamma}\right) \cdot \psi d \mu+\int_{\Gamma} \nabla_{\Gamma}^{\gamma} \cdot \psi d \mu=0
\end{aligned}
$$

on the time interval $(0, \bar{T}]$ and for all $\varphi, \psi: G_{\bar{T}} \rightarrow \mathbb{R}^{n}$. 
Next we observe that using Lemma 4.12 a mixed formulation for the (geometrically equivalent) flow of Problem 3.4 now reads

PRoBlem 4.15 For given initial surface $\Gamma_{0}$ and $u_{0}=i d_{\Gamma_{0}}$ determine $u, \kappa_{\gamma}^{N}: G_{\bar{T}} \rightarrow \mathbb{R}^{n}$ such that $u=i d_{\Gamma}, u(\cdot, 0)=u_{0}$ on $\Gamma_{0}$, and

$$
\begin{aligned}
& \int_{\Gamma} \frac{\dot{u}}{\gamma(v)} \cdot \varphi d V+\frac{1}{2} \int_{\Gamma}\left|\frac{\kappa_{\gamma}^{N}}{\gamma(v)}\right|^{2} \nabla_{\Gamma}^{\gamma} \cdot \varphi d \mu \\
& \quad-\int_{\Gamma}\left|\frac{\kappa_{\gamma}^{N}}{\gamma(v)}\right|^{2} \nabla_{\Gamma} \cdot \varphi d \mu-\int_{\Gamma} \nabla_{\Gamma}^{\gamma} \cdot \kappa_{\gamma}^{N} \nabla_{\Gamma}^{\gamma} \cdot \varphi d \mu \\
& +\int_{\Gamma} \underline{D}_{l}^{\gamma} \varphi^{s} \underline{D}_{s}^{\gamma}\left(\kappa_{\gamma}^{N}\right)^{l} d \mu-\int_{\Gamma} \frac{\nu^{s}}{\gamma(v)} \frac{v^{l}}{\gamma(v)} \gamma_{p_{k}} p_{m}\left(\frac{v}{\gamma(v)}\right) \underline{D}_{m}^{\gamma} \varphi^{s} \underline{D}_{k}^{\gamma}\left(\kappa_{\gamma}^{N}\right)^{l} d \mu=0 \\
& \int_{\Gamma} \frac{\kappa_{\gamma}^{N}}{\gamma(v)} \cdot \psi d V+\int_{\Gamma} \nabla_{\Gamma}^{\gamma} \cdot \psi d \mu=0
\end{aligned}
$$

on the time interval $(0, \bar{T})$ and for all $\varphi, \psi: G_{\bar{T}} \rightarrow \mathbb{R}^{n}$.

In the isotropic case both Problem 4.14 and Problem 4.15 coincide with the formulation presented in [10, Problem 2].

REMARK 4.16 Note that when working with curves in the plane we have that (using (6.1) below)

$$
-\int_{\Gamma} \nabla_{\Gamma}^{\gamma} \cdot \kappa_{\gamma}^{N} \nabla_{\Gamma}^{\gamma} \cdot \varphi d \mu+\int_{\Gamma} \underline{D}_{l}^{\gamma} \varphi^{r} \underline{D}_{r}^{\gamma}\left(\kappa_{\gamma}^{N}\right)^{l} d \mu=0
$$

and the above expression simplifies.

\section{Discretization}

As remarked in Section 3.1 we would like to avoid a numerical scheme containing expressions involving $\gamma, \gamma^{*}$ and their derivatives, hence we will discretize and provide numerical experiments only for Problem 4.15.

As in [10] (cf. also [11]), for a fixed time $t$ we approximate the smooth surface $\Gamma=\Gamma(t)$ by a polyhedral surface $\Gamma_{h}=\Gamma_{h}(t), \Gamma_{h}=\bigcup_{T_{h} \in \mathcal{T}_{h}} T_{h}$, consisting of nondegenerate $(n-1)$-simplices $T_{h}$ that form an admissible triangulation. Let $\mathbb{R}^{n} \ni a_{j}=a_{j}(t)(j=1, \ldots, N)$ be the vertices (nodes) of the triangulation. The finite element space

$$
S_{h}(t)=\left\{\eta \in C^{0}\left(\Gamma_{h}(t)\right):\left.\eta\right|_{T_{h}} \in \mathbb{P}_{1}\left(T_{h}\right), T_{h} \in \mathcal{T}_{h}\right\}
$$

is spanned by the usual nodal basis functions, i.e. $S_{h}(t)=\operatorname{span}\left\{\phi_{1}, \ldots, \phi_{N}\right\}$ with $\phi_{i}\left(a_{j}(t)\right)=\delta_{i j}$. For a function $\eta \in C^{0}\left(\Gamma_{h}(t)\right)$ we denote by $I_{h} \eta \in S_{h}(t)$ the map defined by $I_{h} \eta\left(a_{j}(t)\right)=\eta\left(a_{j}(t)\right)$. Thus we can write $I_{h} \eta=\sum_{j=1}^{N} \eta\left(a_{j}(t)\right) \phi_{j}$.

As in the smooth setting it is useful to consider the topological cylinder $G_{\bar{T}}^{h}=\bigcup_{t \in[0, \bar{T}]} \Gamma_{h}(t) \times$ $\{t\}$. With $u_{h}$ we denote the map $u_{h}: G_{\bar{T}}^{h} \rightarrow \mathbb{R}^{n}$ such that $u_{h}(p, t)=p$. For a local parametrisation $X_{h}$ of $\Gamma_{h}(t)$ we get that $\dot{u}_{h}\left(X_{h}(x, t), t\right)=X_{h t}(x, t)$. With some abuse of notation let $\phi_{i}: G_{\bar{T}}^{h} \rightarrow$ 
$\mathbb{R}, j=1, \ldots, N$ be the map such that $\phi_{i}\left(a_{j}(t), t\right)=\delta_{i j}$. These maps are piecewise linear in space and do not depend on time, therefore $\dot{\phi}_{i}\left(X_{h}(x, t), t\right)=\frac{d}{d t} \phi_{i}\left(X_{h}(x, t), t\right)=0$. We can write $u_{h}(p, t)=\sum_{j=1}^{N} a_{j}(t) \phi_{j}(p, t)$ for $p \in \Gamma_{h}(t)$ and we obtain $X_{h t}(x, t)=\dot{u}_{h}\left(X_{h}(x, t), t\right)=$ $\frac{d}{d t}\left(\sum_{j=1}^{N} a_{j}(t) \phi_{j}\left(X_{h}(x, t), t\right)\right)=\sum_{j=1}^{N} a_{j}^{\prime}(t) \phi_{j}\left(X_{h}(x, t), t\right)$. Therefore the discrete velocity is given by

$$
V_{h}(p, t)=\sum_{j=1}^{N} a_{j}^{\prime}(t) \phi_{j}(p, t), \quad p \in \Gamma_{h}(t) .
$$

The spatial discretization of Problem 4.15 is given as follows.

Problem 5.1 For a given initial value $u_{h 0} \in S_{h}(0)^{n}$ determine $u_{h}(\cdot, t), \kappa_{h}^{N}(\cdot, t) \in S_{h}(t)^{n}$ such that $u_{h}(\cdot, t)=i d_{\Gamma_{h}(t)}$ and

$$
\begin{aligned}
\int_{\Gamma_{h}} \frac{\dot{u}_{h}}{\gamma\left(v_{h}\right)} \cdot \varphi_{h} d V+\frac{1}{2} \int_{\Gamma_{h}} \frac{\left|\kappa_{h}^{N}\right|^{2}}{\gamma\left(v_{h}\right)} \nabla_{\Gamma_{h}}^{\gamma} \cdot \varphi_{h} d V-\int_{\Gamma_{h}} \frac{\left|\kappa_{h}^{N}\right|^{2}}{\gamma\left(v_{h}\right)} \nabla_{\Gamma_{h}} \cdot \varphi_{h} d V \\
-\int_{\Gamma_{h}} \nabla_{\Gamma_{h}}^{\gamma} \cdot \kappa_{h}^{N} \nabla_{\Gamma_{h}}^{\gamma} \cdot \varphi_{h} d \mu+\int_{\Gamma_{h}} \underline{D}_{l}^{\gamma} \varphi_{h}^{s} \underline{D}_{s}^{\gamma}\left(\kappa_{h}^{N}\right)^{l} d \mu \\
\quad-\int_{\Gamma_{h}} \frac{v_{h}^{s}}{\gamma\left(v_{h}\right)} \frac{v_{h}^{l}}{\gamma\left(v_{h}\right)} \gamma_{p_{k} p_{m}}\left(\frac{v_{h}}{\gamma\left(v_{h}\right)}\right) \underline{D}_{m}^{\gamma} \varphi_{h}^{s} \underline{D}_{k}^{\gamma}\left(\kappa_{h}^{N}\right)^{l} d \mu=0 \\
\quad \int_{\Gamma_{h}} \frac{\kappa_{h}^{N}}{\gamma\left(v_{h}\right)} \cdot \psi_{h} d V+\int_{\Gamma_{h}} \nabla_{\Gamma_{h}}^{\gamma} \cdot \psi_{h} d \mu=0
\end{aligned}
$$

on the time interval $(0, \bar{T})$ and for all $\varphi_{h}, \psi_{h} \in S_{h}(t)^{n}$.

Note that the discrete (anisotropic) tangential gradient and divergence are understood piecewise on each simplex.

As in the smooth setting, we can show that the energy decreases along the flow.

Lemma 5.2 Assume that $\left(u_{h}, \kappa_{h}^{N}\right)$ is a solution of Problem 5.1. Let

$$
W_{h}\left(u_{h}\right):=\frac{1}{2} \int_{\Gamma_{h}} \frac{\left|\kappa_{h}^{N}\right|^{2}}{\gamma\left(v_{h}\right)} d V
$$

be the discrete anisotropic Willmore functional. Then the energy relation

$$
\int_{\Gamma_{h}} \frac{\left|\dot{u}_{h}\right|^{2}}{\gamma\left(v_{h}\right)} d V+\frac{d}{d t} W_{h}\left(u_{h}\right)=0
$$

holds for all times in $(0, \bar{T})$.

Proof. First of all note that the first equation in Problem 5.1 is equal to

$$
\int_{\Gamma_{h}} \frac{\dot{u}_{h}}{\gamma\left(v_{h}\right)} \cdot \varphi_{h} d V+\left\langle W_{h}^{\prime}\left(u_{h}\right), \varphi_{h}\right\rangle=0 .
$$


To prove the above statement one can repeat the arguments used for the computation of the first variation of the Willmore functional (see Section 4.3). Note that all steps are still meaningful in the discrete setting, since no integration by parts was used.

Finally, the claim is achieved by choosing $\varphi_{h}=\dot{u}_{h}$. Note that $\dot{u}_{h}$ is an admissible function since by definition $u_{h}=i d_{\Gamma_{h}}$ and as remarked above $\dot{u}_{h}\left(X_{h}(x, t)\right)=X_{h t}(x, t)=$ $\sum_{j=1}^{N} a_{j}^{\prime}(t) \phi_{j}\left(X_{h}(x, t), t\right) \in S_{h}(t)^{n}$.

When dealing with curves we will find it more convenient to work with the interpolation operator (similarly also to the isotropic case studied in [7]). Thus we define

Problem 5.3 For a given initial value $u_{h 0} \in S_{h}(0)^{n}$ determine $u_{h}(\cdot, t), \kappa_{h}^{N}(\cdot, t) \in S_{h}(t)^{n}$ such that $u_{h}(\cdot, t)=i d_{\Gamma_{h}(t)}$ and

$$
\begin{aligned}
& \int_{\Gamma_{h}} I_{h}\left(\frac{\dot{u}_{h}}{\gamma\left(v_{h}\right)} \cdot \varphi_{h}\right) d V+\frac{1}{2} \int_{\Gamma_{h}} I_{h}\left(\frac{\left|\kappa_{h}^{N}\right|^{2}}{\gamma\left(v_{h}\right)}\right) \nabla_{\Gamma_{h}}^{\gamma} \cdot \varphi_{h} d V \\
& -\int_{\Gamma_{h}} I_{h}\left(\frac{\left|\kappa_{h}^{N}\right|^{2}}{\gamma\left(v_{h}\right)}\right) \nabla_{\Gamma_{h}} \cdot \varphi_{h} d V-\int_{\Gamma_{h}} \nabla_{\Gamma_{h}}^{\gamma} \cdot \kappa_{h}^{N} \nabla_{\Gamma_{h}}^{\gamma} \cdot \varphi_{h} d \mu \\
& \quad+\int_{\Gamma_{h}} \underline{D}_{l}^{\gamma} \varphi_{h}^{s} \underline{D}_{s}^{\gamma}\left(\kappa_{h}^{N}\right)^{l} d \mu-\int_{\Gamma_{h}} \frac{v_{h}^{s}}{\gamma\left(v_{h}\right)} \frac{v_{h}^{l}}{\gamma\left(v_{h}\right)} \gamma_{p_{k} p_{m}}\left(\frac{v_{h}}{\gamma\left(v_{h}\right)}\right) \underline{D}_{m}^{\gamma} \varphi_{h}^{s} \underline{D}_{k}^{\gamma}\left(\kappa_{h}^{N}\right)^{l} d \mu=0, \\
& \int_{\Gamma_{h}} I_{h}\left(\frac{\kappa_{h}^{N}}{\gamma\left(v_{h}\right)} \cdot \psi_{h}\right) d V+\int_{\Gamma_{h}} \nabla_{\Gamma_{h}}^{\gamma} \cdot \psi_{h} d \mu=0
\end{aligned}
$$

on the time interval $(0, \bar{T})$ and for all $\varphi_{h}, \psi_{h} \in S_{h}(t)^{n}$.

Using the arguments employed for the proof of the analogous Lemma 5.2, the fact that $\gamma\left(v_{h}\right)$ is a constant function on each simplex, and the linearity of the interpolation operator, we infer that

LEMmA 5.4 Assume that $\left(u_{h}, \kappa_{h}^{N}\right)$ is a solution of Problem 5.3. Let

$$
W_{h}\left(u_{h}\right):=\frac{1}{2} \int_{\Gamma_{h}} I_{h}\left(\frac{\left|\kappa_{h}^{N}\right|^{2}}{\gamma\left(v_{h}\right)}\right) d V=\frac{1}{2} \int_{\Gamma_{h}} I_{h}\left(\left|\frac{\kappa_{h}^{N}}{\gamma\left(v_{h}\right)}\right|^{2}\right) \gamma\left(v_{h}\right) d V
$$

be the discrete anisotropic Willmore functional. Then the energy relation

$$
\int_{\Gamma_{h}} I_{h}\left(\frac{\left|\dot{u}_{h}\right|^{2}}{\gamma\left(v_{h}\right)}\right) d V+\frac{d}{d t} W_{h}\left(u_{h}\right)=0
$$

holds for all times in $(0, \bar{T})$.

\section{Fully discrete numerical schemes}

The time discretization of the anisotropic Willmore flow is a challenging task. In theory there are many possible different approaches and it is difficult to tell a priori which scheme will be successful.

Our goal is to provide semi-implicit schemes that treats nonlinearities in an explicit way. This allows for a straightforward implementation (basically a linear system has to be solved in each time 
step); however we are bound to pay in terms of stability, as the experience with the anisotropic mean curvature flow has shown us (cf. for instance [16], [17], where we had to introduce a stability term in order to make the discretization a stable one).

Note that since the mixed formulation for the anisotropic Willmore flow basically contains a formulation for the anisotropic mean curvature flow, we really are faced with two non-trivial problems: we have to deal with the possible instability of the anisotropic mean curvature component and, simultaneously, we have to make sure to provide a good time discretization for the anisotropic Willmore flow.

\subsection{The curve case}

We start with the curve case, since its simpler structure allows for some elementary analysis and heuristics that proves helpful in identifying good discretization strategies.

Since curves can be parametrized over the fixed domain $S^{1}=[0,2 \pi]$, we start by reviewing briefly the anisotropic operators identified in Section 4.1.3 in terms of this parametrization. More precisely, for a regular curve $u: S^{1} \rightarrow \mathbb{R}^{2}, u=u(x)$, with euclidean unit tangent $\tau=\frac{u_{x}}{\left|u_{x}\right|}=u_{s}$, where $s$ denotes the arc-length parameter (hence $d s=\left|u_{x}\right| d x, \partial_{s}=\frac{1}{\left|u_{x}\right|} \partial_{x}$ ), and Euclidean unit normal $\nu$, we have $\nabla_{\Gamma} f=\left(\partial_{s} f\right) \tau$ and

$$
\underline{D}_{m}^{\gamma} f=\partial_{s} f\left(\tau^{m}-\left(\gamma^{\prime}(v) \cdot \tau\right) \frac{\nu^{m}}{\gamma(\nu)}\right)
$$

(recall (4.11) and (4.15)). Note that for simplicity of notation we use here the same notation for a map $f: \Gamma=u\left(S^{1}\right) \rightarrow \mathbb{R}$ and $f \circ u: S^{1} \rightarrow \mathbb{R}$. For a vector field $\psi: \Gamma \rightarrow \mathbb{R}^{2}$, we infer from (4.19) that

$$
\nabla_{\Gamma}^{\gamma} \cdot \psi=\underline{D}_{m}^{\gamma} \psi^{m}=\partial_{s} \psi \cdot\left(\tau-\left(\tau \cdot \gamma^{\prime}(v)\right) \frac{v}{\gamma(\nu)}\right)=\partial_{s} \psi \cdot \tilde{P}^{\gamma} \tau
$$

REMARK 6.1 Note that another expression for (6.2) is obtained using (4.20) as follows

$$
\begin{aligned}
\nabla_{\Gamma}^{\gamma} \cdot \psi= & \underline{D}_{j}^{\gamma}\left(I d^{i}\right) \underline{D}_{i}^{\gamma} \psi^{j} \\
= & \partial_{s} u^{i}\left(\tau^{j}-\left(\gamma^{\prime}(v) \cdot \tau\right) \frac{v^{j}}{\gamma(v)}\right) \partial_{s} \psi^{j}\left(\tau^{i}-\left(\gamma^{\prime}(v) \cdot \tau\right) \frac{v^{i}}{\gamma(v)}\right) \\
= & \left(\partial_{s} u \cdot \tau\right)\left(\tau \cdot \partial_{s} \psi\right)-\left(\gamma^{\prime}(v) \cdot \tau\right)\left(\partial_{s} u \cdot \frac{v}{\gamma(v)}\right)\left(\tau \cdot \partial_{s} \psi\right)-\left(\gamma^{\prime}(v) \cdot \tau\right)\left(\partial_{s} \psi \cdot \frac{v}{\gamma(v)}\right)\left(\tau \cdot \partial_{s} u\right) \\
& \quad+\left(\gamma^{\prime}(v) \cdot \tau\right)^{2}\left(\partial_{s} \psi \cdot \frac{v}{\gamma(v)}\right)\left(\partial_{s} u \cdot \frac{v}{\gamma(v)}\right) .
\end{aligned}
$$

Of course equations (6.2) and (6.3) are analytically identical, since obviously

$$
\partial_{s} u=\tau, \quad\left(\partial_{s} u \cdot \tau\right)=1, \quad\left(\partial_{s} u \cdot v\right)=0 .
$$

However if we use a time discretization which treats $\partial_{s} u$ and $\tau$ as different objects, i.e. if we consider $\partial_{s} u$ and $\tau$ as approximations of the same map but at different time levels, (for instance by taking $u$ implicit and $\tau$ explicit, that is by considering $\partial_{s} u$ as approximation of the unit tangent at time 
$t_{m+1}=(m+1) \delta$ and $\tau$ at time $\left.t_{m}=\delta m\right)$ then (6.4) no longer holds and we might introduce several errors in the discrete schema.

This phenomenon can be observed very well in the isotropic case. Here, since $\gamma(v)=1, \gamma^{\prime}(v)=$ $v$, and $\nabla_{\Gamma}^{\gamma}=\nabla_{\Gamma}$, we infer for (6.2) that

$$
\nabla_{\Gamma} \cdot \psi=\underline{D}_{m} \psi^{m}=\partial_{s} \psi \cdot \tau
$$

Moreover from (6.3) and using (4.22), we can equivalently write

$$
\nabla_{\Gamma} \cdot \psi=\underline{D}_{j}\left(I d^{i}\right) \underline{D}_{i} \psi^{j}=\underline{D}_{i}\left(I d^{j}\right) \underline{D}_{i} \psi^{j}=\nabla_{\Gamma}(I d): \nabla_{\Gamma} \psi=\partial_{s} \psi \cdot \partial_{s} u
$$

In practice (6.6) is the expression used for example to provide a stable discretization for the curve shortening flow: here one takes the map $u$ implicit (see [8, (4.14)]) .

On the other hand (6.3) gives (without any sort of manipulation)

$$
\nabla_{\Gamma} \cdot \psi=\underline{D}_{j}\left(I d^{i}\right) \underline{D}_{i} \psi^{j}=\left(\partial_{s} u \cdot \tau\right)\left(\tau \cdot \partial_{s} \psi\right) .
$$

If we stick to making $u$ implicit and $\tau$ explicit then we see that we obtain quite a different scheme and it is a priori not clear why (6.7) should be as good as (6.6). In fact, in addition to the nasty coupling of the equations for different space components (recall $\underline{D}_{j}\left(I d^{i}\right) \underline{D}_{i} \psi^{j}$ ), we loose the beautiful linear structure of (6.6).

In view of these considerations we "discard" formulation (6.3) (in spite of it being analytically very appealing, since we use the same anisotropic operator $\nabla_{\Gamma}^{\gamma}(\cdot)$ on both maps $I d$ and $\psi$ ) and concentrate on expression (6.2). Also in this case we still have several possibilities regarding the choices of explicit and implicit terms. In this paper we will study schemes corresponding to

$$
\nabla_{\Gamma}^{\gamma} \cdot \psi=\partial_{s} \psi \cdot\left(\partial_{s} u-\left(\tau \cdot \gamma^{\prime}(v)\right) \frac{v}{\gamma(v)}\right)
$$

where as above $u$ refers to the quantity treated implicitly. Note that this scheme corresponds to the one for anisotropic mean curvature flow of surfaces ( $[17,(6.1)],[6, \S 4.2])$, which has proved experimentally to be unstable.

The expression for the anisotropic Willmore flow of curves simplifies if we consider $\phi: \mathbb{R}^{2} \rightarrow$ $[0, \infty)$ such that $\phi(\tau)=\gamma(v)$, with $v=\tau^{\perp}\left(\right.$ where $\left.\left(a_{1}, a_{2}\right)^{\perp}=\left(-a_{2}, a_{1}\right)\right)$ and write all integrals in terms of $\phi$. With this in mind, observe that from $\phi(\tau)=\gamma(\nu)$ we infer

$$
\gamma^{\prime}(v)=\phi^{\prime}(\tau)^{\perp}, \quad \phi^{\prime \prime}(\tau) v \cdot v=\gamma^{\prime \prime}(v) \tau \cdot \tau,
$$

as well as (recall also (2.4))

$$
\left(\gamma^{\prime \prime}(\nu) \tau \cdot \tau\right)\left(\partial_{s} \varphi \cdot v\right)\left(\partial_{s} \kappa_{\gamma}^{N} \cdot v\right)=\phi^{\prime \prime}(\tau)\left(\partial_{s} \varphi \cdot v\right) v \cdot\left(\partial_{s} \kappa_{\gamma}^{N} \cdot v\right) v=\phi^{\prime \prime}(\tau) \partial_{s} \varphi \cdot \partial_{s} \kappa_{\gamma}^{N} .
$$

Moreover $\phi^{\prime}(\tau)=\phi^{\prime}\left(u_{x}\right), \phi^{\prime \prime}(\tau)=\left|u_{x}\right| \phi^{\prime \prime}\left(u_{x}\right)$, and $v \cdot \phi^{\prime}(\tau)=-\tau \cdot \gamma^{\prime}(v)$. Finally, using $\phi(\tau)=$ $\gamma(\nu), \phi(\tau)=\phi^{\prime}(\tau) \cdot \tau,(6.8)$, and taking $\sigma=1$ we can write

$$
\begin{aligned}
\nabla_{\Gamma}^{\gamma} \cdot \psi \phi(\tau) & =\phi(\tau) \partial_{s} \psi \cdot\left(\partial_{s} u-\tau\right)+\left[\left(\phi^{\prime}(\tau) \cdot \tau\right) \tau+\left(\phi^{\prime}(\tau) \cdot \nu\right) \nu\right] \cdot \partial_{s} \psi \\
& =\sigma \phi(\tau) \partial_{s} \psi \cdot\left(\partial_{s} u-\tau\right)+\left(\partial_{s} \psi \cdot \phi^{\prime}(\tau)\right) .
\end{aligned}
$$


Note that in the isotropic case we recover $\nabla_{\Gamma}^{\gamma} \cdot \psi \phi(\tau)=\partial_{s} \psi \cdot \partial_{s} u$. The idea exploited in [17] and [16] for the anisotropic mean curvature flow was to choose some $\sigma \geqslant 1$ (determined by the value of some higher norms of $\phi$ ), so that stability could be obtained for the discrete flow. We will do something similar in what follows.

Replacing first $\gamma$ with $\phi$ as remarked above, using (6.10), (6.6), (6.1), (6.9), and Remark 4.16 we can write Problem 4.15 as

Problem 6.2 Let $\lambda=0$ and $\sigma=\tilde{\sigma}=\hat{\sigma}=1$. For a given initial curve $u_{0}: S^{1} \rightarrow \mathbb{R}^{2}$, determine $u, \kappa_{\gamma}^{N}: S^{1} \times[0, T) \rightarrow \mathbb{R}^{2}$ such that $u(\cdot, 0)=u_{0}$ and

$$
\begin{aligned}
& 0=\int_{S^{1}} \frac{u_{t}}{\phi(\tau)} \cdot \varphi d s+\tilde{\sigma} \frac{1}{2} \int_{S^{1}}\left|\frac{\kappa_{\gamma}^{N}}{\phi(\tau)}\right|^{2} \partial_{s} \varphi \cdot\left(\partial_{s} u-\tau\right) \phi(\tau) d s+\frac{1}{2} \int_{S^{1}}\left|\frac{\kappa_{\gamma}^{N}}{\phi(\tau)}\right|^{2} \partial_{s} \varphi \cdot \phi^{\prime}(\tau) d s \\
& -\int_{S^{1}}\left|\frac{\kappa_{\gamma}^{N}}{\phi(\tau)}\right|^{2} \partial_{s} \varphi \cdot \partial_{s} u \phi(\tau) d s-\int_{S^{1}} \phi^{\prime \prime}(\tau) \partial_{s} \varphi \cdot \partial_{s} \kappa_{\gamma}^{N} d s \\
& +\lambda\left(\hat{\sigma} \int_{S^{1}} \partial_{s} \varphi \cdot\left(\partial_{s} u-\tau\right) \phi(\tau) d s+\int_{S^{1}} \phi^{\prime}(\tau) \cdot \varphi_{s} d s\right), \\
& 0=\int_{S^{1}} \frac{\kappa_{\gamma}^{N}}{\phi(\tau)} \cdot \psi d s+\sigma \int_{S^{1}} \partial_{s} \psi \cdot\left(\partial_{s} u-\tau\right) \phi(\tau) d s+\int_{S^{1}} \phi^{\prime}(\tau) \cdot \psi_{s} d s
\end{aligned}
$$

on the time interval $(0, T)$ and for all test functions $\varphi, \psi: S^{1} \rightarrow \mathbb{R}^{2}$.

The term multiplying $\lambda$ has been introduced as a term penalizing the growth of the anisotropic length of the curve: in other words if we choose $\lambda>0$ we are considering a gradient flow for the energy

$$
E(u)=\frac{1}{2} \int_{S^{1}} \frac{\left|\kappa_{\gamma}^{N}\right|^{2}}{\phi(\tau)} d s+\lambda \int_{S^{1}} \phi(\tau) d s=W_{\gamma}(u)+\lambda A_{\gamma}(u)
$$

where, as usual, $\gamma(v)=\phi(\tau)$. A motivation for penalizing the length growth is given by Example 3.1.

We now discretize in space and time. We use the notation $v^{m}=v(\cdot, m \delta)$ for the discrete time levels with time step $\delta>0$ and $m=0,1, \ldots, m_{T}$, where $m_{T} \delta=T$. A discretization of the above Problem 6.2 in the spirit of Problem 5.3 (where now we write $\kappa_{h}$ instead of $\kappa_{h}^{N}$ for simplicity of notation) and leaving now freedom in the choice of the positive parameter $\lambda, \sigma, \hat{\sigma}$, and $\tilde{\sigma}$, is given by

Problem 6.3 Let $\lambda \geqslant 0$ and $\sigma, \tilde{\sigma}, \hat{\sigma} \geqslant 1$. For a given initial polygonal curve $u^{0}=I_{h}\left(u_{0}\right)$, and time step $\delta$, determine for $m=0, \ldots, m_{T}-1$ piecewise linear continuous maps $u_{h}^{m+1}, \kappa_{h}^{m+1}$ such that

$$
\begin{aligned}
0=\int_{S^{1}} & I_{h}\left(\frac{u_{h}^{m+1}-u_{h}^{m}}{\delta \phi\left(\tau_{h}^{m}\right)} \cdot \varphi_{h}\right)\left|u_{h x}^{m}\right| d x+\frac{\tilde{\sigma}}{2} \int_{S^{1}} I_{h}\left(\frac{\left|\kappa_{h}^{m}\right|^{2}}{\phi\left(\tau_{h}^{m}\right)}\right) \varphi_{h x} \cdot \frac{u_{h x}^{m+1}-u_{h x}^{m}}{\left|u_{h x}^{m}\right|} d x \\
& +\frac{1}{2} \int_{S^{1}} I_{h}\left(\frac{\left|\kappa_{h}^{m}\right|^{2}}{\left|\phi\left(\tau_{h}^{m}\right)\right|^{2}}\right) \varphi_{h x} \cdot \phi^{\prime}\left(\tau_{h}^{m}\right) d x-\int_{S^{1}} I_{h}\left(\frac{\left|\kappa_{h}^{m}\right|^{2}}{\phi\left(\tau_{h}^{m}\right)}\right) \varphi_{h x} \cdot \frac{u_{h x}^{m+1}}{\left|u_{h x}^{m}\right|} d x
\end{aligned}
$$




$$
\begin{gathered}
-\int_{S^{1}}\left(\phi^{\prime \prime}\left(\tau_{h}^{m}\right) \varphi_{h x} \cdot \frac{\kappa_{h x}^{m+1}}{\left|u_{h x}^{m}\right|}\right) d x \\
+\lambda\left(\hat{\sigma} \int_{S^{1}} \varphi_{h x} \cdot \frac{u_{h x}^{m+1}-u_{h x}^{m}}{\left|u_{h x}^{m}\right|} \phi\left(\tau_{h}^{m}\right) d x+\int_{S^{1}} \varphi_{h x} \cdot \phi^{\prime}\left(\tau_{h}^{m}\right) d x\right), \\
0=\int_{S^{1}} I_{h}\left(\frac{\kappa_{h}^{m+1}}{\phi\left(\tau_{h}^{m}\right)} \cdot \psi\right)\left|u_{h x}^{m}\right| d x+\sigma \int_{S^{1}} \psi_{h x} \cdot \frac{u_{h x}^{m+1}-u_{h x}^{m}}{\left|u_{h x}^{m}\right|} \phi\left(\tau_{h}^{m}\right) d x+\int_{S^{1}} \psi_{h x} \cdot \phi^{\prime}\left(\tau_{h}^{m}\right) d x
\end{gathered}
$$

for all (piecewise linear and globally continuos) test functions $\varphi_{h}, \psi_{h}: S^{1} \rightarrow \mathbb{R}^{2}$.

Note that in the isotropic setting $(\phi(x)=|x|)$ and with $1=\sigma=\hat{\sigma}=\tilde{\sigma}$ we recover the discretization presented in [7] for the (isotropic) elastic flow of curves (in this paper an error analysis for the semi-discrete scheme is also derived).

REMARK 6.4 Here we try to motivate the introduction of the (stability) parameters $\tilde{\sigma}$ and $\sigma$ (analogous considerations can be made for $\hat{\sigma}$ ). For simplicity, consider the flow without any constraint on the length, thus $\lambda=0$ (so that $\hat{\sigma}$ plays no role). In the above scheme the parameter $\sigma$ and $\tilde{\sigma}$ should be thought of as

$$
\sigma=1+\mu, \quad \tilde{\sigma}=1+\tilde{\mu}
$$

where $\mu, \tilde{\mu} \geqslant 0$ must be chosen appropriately. The $\sigma$-parameter is related to the stability of the anisotropic mean curvature component. Numerical experiments (see Section 6.1.1 below) show that it is best to choose $\sigma=1$. A heuristic for the isotropic Willmore flow of curves indicates that a choice of $\tilde{\mu} \geqslant 1$ might help making the isotropic elastic flow more stable. Numerical tests in isotropic and anisotropic setting (see Section 6.1.1 below) further support the claim that stability is achieved by appropriate choices of $\tilde{\mu}$.

Of course the addition of the $\tilde{\mu}$-term modifies the flow, however it can be thought of as a term approximating

$$
\frac{\delta}{2} \int_{S^{1}} I_{h}\left(\frac{\left|\kappa_{h}^{m}\right|^{2}}{\phi\left(\tau_{h}^{m}\right)}\right) \varphi_{h x} \cdot \frac{u_{h x}^{m+1}-u_{h x}^{m}}{\delta\left|u_{h x}^{m}\right|} d x \simeq \frac{\delta}{2} \int_{S^{1}} I_{h}(\ldots) \varphi_{s} \cdot u_{t s} d s
$$

which, under suitable assumptions on the velocity of $u_{t}$, should "disappear" as we make the time step smaller $\delta \rightarrow 0$.

The heuristic mentioned above for the choice of this stability term is based on the derivation of the energy estimates in the smooth (or semi-discrete) and fully discrete setting of the isotropic elastic flow (cf. also [7, Remark 2.1(b)]). In the smooth setting the verification that the energy decreases along the flow can be obtained by taking $\varphi=u_{t}$ in $(E 1)$ and $\psi=\kappa_{\gamma}^{N}$ in the equation $(E 2)$ after derivation with respect to time, thus in $(E 2)_{t}$ (cf. Problem 6.2 in the isotropic setting with $\sigma=\tilde{\sigma}=1, \lambda=0, \phi(\tau)=1)$. The discrete approximation of $(E 2)_{t}$, which is obtained by subtracting the discrete equation for $\kappa_{h}$ at two subsequent time-steps, does not quite reproduce the above term. Hence the stability term can be interpreted as an attempt to make up for this failure.

We now make more precise the notation used for the discretization given in Problem 6.3. Let $0=x_{0}<x_{1}<\ldots<x_{N}=2 \pi$ be a partition of $[0,2 \pi]$ and $h_{j}:=x_{j}-x_{j-1}$ as well as $h:=\max _{j=1, \ldots, N} h_{j}$. The piecewise linear continuous maps $u_{h}^{m}, \kappa_{h}^{m}: S^{1} \rightarrow \mathbb{R}^{2}$ can be described 
as follows

$$
\left(u_{h}^{m}\right)_{i}(x)=\sum_{j=1}^{N} u_{j, i}^{m} \varphi_{j}(x), \quad\left(\kappa_{h}^{m}\right)_{i}(x)=\sum_{j=1}^{N} k_{j, i}^{m} \varphi_{j}(x), \quad(i=1,2),
$$

where $\varphi_{j}: S^{1} \rightarrow \mathbb{R}$ is the piecewise linear nodal basis function with $\varphi_{j}\left(x_{r}\right)=\delta_{r j},(r, j=$ $1, \ldots N), u_{j}^{m}=\left(u_{j, 1}^{m}, u_{j, 2}^{m}\right), k_{j}^{m}=\left(k_{j, 1}^{m}, k_{j, 2}^{m}\right) \in \mathbb{R}^{2}, j=1, \ldots, N$, and where periodicity conditions hold (i.e. $u_{0}^{m}=u_{N}^{m}, u_{1}^{m}=u_{N+1}^{m}, k_{0}^{m}=k_{N}^{m}, k_{1}^{m}=k_{N+1}^{m}$ ). We have

$$
\begin{aligned}
& q_{j}^{m}:=\left|u_{j}^{m}-u_{j-1}^{m}\right|, \quad\left|u_{h x}^{m}(x)\right|=\frac{q_{j}^{m}}{h_{j}} \quad x \in\left[x_{j-1}, x_{j}\right], \\
& \tau_{h}^{m}(x)=\tau_{j}^{m}=\frac{u_{j}^{m}-u_{j-1}^{m}}{\left|u_{j}^{m}-u_{j-1}^{m}\right|}, \quad v_{h}^{m}(x)=v_{j}^{m}=\left(\tau_{j}^{m}\right)^{\perp}, \quad x \in\left[x_{j-1}, x_{j}\right],
\end{aligned}
$$

together with the periodicity conditions $\tau_{N+1}^{m}=\tau_{1}^{m}, v_{1}^{m}=v_{N+1}^{m}$, and $q_{1}^{m}=q_{N+1}^{m}$. Moreover we write

$$
\alpha_{j}:=\frac{\phi\left(\tau_{j}^{m}\right)}{q_{j}^{m}}, \quad m_{j}:=\frac{\left|k_{j-1}^{m}\right|^{2}+\left|k_{j}^{m}\right|^{2}}{4 q_{j}^{m} \phi\left(\tau_{j}^{m}\right)} \quad(j=1, \ldots, N),
$$

(where we have omitted the time indices for better readability) with periodicity conditions $\alpha_{1}=$ $\alpha_{N+1}$, and $m_{N+1}=m_{1}$. Problem 6.3 yields the following system (for $j=1, \ldots, N$ ):

$$
\begin{aligned}
& \frac{1}{2 \delta}\left(\frac{1}{\alpha_{j}}+\frac{1}{\alpha_{j+1}}\right) u_{j}^{m}+\tilde{\sigma}\left(q_{j}^{m} m_{j} \tau_{j}^{m}-q_{j+1}^{m} m_{j+1} \tau_{j+1}^{m}\right)-\frac{\phi^{\prime}\left(\tau_{j}^{m}\right)}{\phi\left(\tau_{j}^{m}\right)} q_{j}^{m} m_{j}+\frac{\phi^{\prime}\left(\tau_{j+1}^{m}\right)}{\phi\left(\tau_{j+1}^{m}\right)} q_{j+1}^{m} m_{j+1} \\
& +\lambda\left(-\phi^{\prime}\left(\tau_{j}^{m}\right)+\phi^{\prime}\left(\tau_{j+1}^{m}\right)\right)+\lambda \hat{\sigma}\left(-\phi\left(\tau_{j+1}^{m}\right) \tau_{j+1}^{m}+\phi\left(\tau_{j}^{m}\right) \tau_{j}^{m}\right) \\
& =\left[\frac{1}{2 \delta}\left(\frac{1}{\alpha_{j}}+\frac{1}{\alpha_{j+1}}\right)+(\tilde{\sigma}-2)\left(m_{j}+m_{j+1}\right)+\lambda \hat{\sigma}\left(\alpha_{j}+\alpha_{j+1}\right)\right] u_{j}^{m+1} \\
& \left.\left.-\left[(\tilde{\sigma}-2) m_{j}+\lambda \hat{\sigma} \alpha_{j}\right)\right] u_{j-1}^{m+1}-\left[(\tilde{\sigma}-2) m_{j+1}+\lambda \hat{\sigma} \alpha_{j+1}\right)\right] u_{j+1}^{m+1} \\
& +\frac{\phi^{\prime \prime}\left(\tau_{j}^{m}\right)}{q_{j}^{m}} k_{j-1}^{m+1}-\left(\frac{\phi^{\prime \prime}\left(\tau_{j}^{m}\right)}{q_{j}^{m}}+\frac{\phi^{\prime \prime}\left(\tau_{j+1}^{m}\right)}{q_{j+1}^{m}}\right) k_{j}^{m+1}+\frac{\phi^{\prime \prime}\left(\tau_{j+1}^{m}\right)}{q_{j+1}^{m}} k_{j+1}^{m+1}, \\
& \sigma\left(-\phi\left(\tau_{j+1}^{m}\right) \tau_{j+1}^{m}+\phi\left(\tau_{j}^{m}\right) \tau_{j}^{m}\right)-\phi^{\prime}\left(\tau_{j}^{m}\right)+\phi^{\prime}\left(\tau_{j+1}^{m}\right) \\
& =\frac{1}{2}\left(\frac{1}{\alpha_{j}}+\frac{1}{\alpha_{j+1}}\right) k_{j}^{m+1}+\sigma\left(\alpha_{j}+\alpha_{j+1}\right) u_{j}^{m+1}-\sigma \alpha_{j} u_{j-1}^{m+1}-\sigma \alpha_{j+1} u_{j+1}^{m+1} .
\end{aligned}
$$

Setting

$$
\begin{aligned}
& \left(\operatorname{RHSK}(j)_{1}, \operatorname{RHSK}(j)_{2}\right)=\sigma\left(-\phi\left(\tau_{j+1}^{m}\right) \tau_{j+1}^{m}+\phi\left(\tau_{j}^{m}\right) \tau_{j}^{m}\right)-\phi^{\prime}\left(\tau_{j}^{m}\right)+\phi^{\prime}\left(\tau_{j+1}^{m}\right) \in \mathbb{R}^{2}, \\
& \left(\operatorname{RHSU}(j)_{1}, \operatorname{RHSU}(j)_{2}\right)=\frac{1}{2 \delta}\left(\frac{1}{\alpha_{j}}+\frac{1}{\alpha_{j+1}}\right) u_{j}^{m}+\tilde{\sigma}\left(q_{j}^{m} m_{j} \tau_{j}^{m}-q_{j+1}^{m} m_{j+1} \tau_{j+1}^{m}\right) \\
& \quad-\frac{\phi^{\prime}\left(\tau_{j}^{m}\right)}{\phi\left(\tau_{j}^{m}\right)} q_{j}^{m} m_{j}+\frac{\phi^{\prime}\left(\tau_{j+1}^{m}\right)}{\phi\left(\tau_{j+1}^{m}\right)} q_{j+1}^{m} m_{j+1} \\
& \quad+\lambda\left(-\phi^{\prime}\left(\tau_{j}^{m}\right)+\phi^{\prime}\left(\tau_{j+1}^{m}\right)\right)+\lambda \hat{\sigma}\left(-\phi\left(\tau_{j+1}^{m}\right) \tau_{j+1}^{m}+\phi\left(\tau_{j}^{m}\right) \tau_{j}^{m}\right) \in \mathbb{R}^{2}, \quad(j=1 \ldots, N)
\end{aligned}
$$


and

$$
\begin{array}{rlr}
\mathbf{u}_{r}=\left(u_{1, r}, \ldots, u_{N, r}\right), & \mathbf{k}_{r}=\left(k_{1, r}, \ldots, k_{N, r}\right), & (r \in\{1,2\}) \\
\mathbf{v}_{r}=\left(R H S U(1)_{r}, \ldots, R H S U(N)_{r}\right), & \mathbf{w}_{r}=\left(\operatorname{RHSK}(1)_{r}, \ldots, R H S K(N)_{r}\right),
\end{array}
$$

as well as symmetric $(N \times N)$-matrices

$$
\begin{aligned}
& M=\operatorname{diag}\left(\frac{1}{2}\left(\frac{1}{\alpha_{j}}+\frac{1}{\alpha_{j+1}}\right)\right), \\
& S=\operatorname{tridiag}\left(-\alpha_{j}, \alpha_{j}+\alpha_{j+1},-\alpha_{j+1}\right), \\
& R=\operatorname{tridiag}\left(-m_{j}, m_{j}+m_{j+1},-m_{j+1}\right), \\
& \Phi_{r l}=\operatorname{tridiag}\left(-\frac{1}{q_{j}^{m}} \phi_{p_{r} p_{l}}\left(\tau_{j}^{m}\right), \frac{1}{q_{j}^{m}} \phi_{p_{r} p_{l}}\left(\tau_{j}^{m}\right)+\frac{1}{q_{j+1}^{m}} \phi_{p_{r} p_{l}}\left(\tau_{j+1}^{m}\right),-\frac{1}{q_{j+1}^{m}} \phi_{p_{r} p_{l}}\left(\tau_{j+1}^{m}\right)\right),
\end{aligned}
$$

where tridiag denotes a tridiagonal matrix except for two entries in the last column of the first row and in the first column of the last row, which are due to periodicity, then we can write

$$
\begin{aligned}
\mathbf{v}_{r} & =\frac{1}{\delta} M \mathbf{u}_{r}^{m+1}+(\tilde{\sigma}-2) R \mathbf{u}_{r}^{m+1}+\lambda \hat{\sigma} S \mathbf{u}_{r}^{m+1}-\sum_{l=1}^{2} \Phi_{r l} \mathbf{k}_{l}^{m+1}, \\
\mathbf{w}_{r} & =M \mathbf{k}_{r}^{m+1}+\sigma S \mathbf{u}_{r}^{m+1}
\end{aligned}
$$

with $r=1,2$. We can eliminate $\mathbf{k}$ from the first equation and obtain

$$
\mathbf{v}_{r}+\sum_{l=1}^{2} \Phi_{r l} M^{-1} \mathbf{w}_{l}=\frac{1}{\delta} M \mathbf{u}_{r}^{m+1}+(\tilde{\sigma}-2) R \mathbf{u}_{r}^{m+1}+\lambda \hat{\sigma} S \mathbf{u}_{r}^{m+1}+\sigma \sum_{l=1}^{2} \Phi_{r l} M^{-1} S \mathbf{u}_{l}^{m+1} .
$$

This gives a system

$$
\left(\begin{array}{c|c}
A & B \\
\hline B & C
\end{array}\right)\left(\begin{array}{c}
\mathbf{u}_{1}^{m+1} \\
\mathbf{u}_{2}^{m+1}
\end{array}\right)=\left(\begin{array}{c}
\mathbf{v}_{1}+\Phi_{11} M^{-1} \mathbf{w}_{1}+\Phi_{12} M^{-1} \mathbf{w}_{2} \\
\mathbf{v}_{2}+\Phi_{21} M^{-1} \mathbf{w}_{1}+\Phi_{22} M^{-1} \mathbf{w}_{2}
\end{array}\right)
$$

where $A=\frac{1}{\delta} M+(\tilde{\sigma}-2) R+\lambda \hat{\sigma} S+\sigma \Phi_{11} M^{-1} S, B=\sigma \Phi_{12} M^{-1} S=\sigma \Phi_{21} M^{-1} S$, and $C=\frac{1}{\delta} M+(\tilde{\sigma}-2) R+\lambda \hat{\sigma} S+\sigma \Phi_{22} M^{-1} S$. The vectors $\mathbf{k}_{r}^{0}, r=1,2$, are computed using the initial data by solving $\mathbf{w}_{r}=M \mathbf{k}_{r}^{0}$ with $\sigma=0$ in $\mathbf{w}_{r}$. We solve the linear system with the BICGstab-method.

6.1.1 Numerical tests for curves in the plane. In the tests that follow we have parametrized all initial curves over the interval $[0,2 \pi]$ and have used a uniform grid. Thus the grid parameter $h$ is given by $h=2 \pi / n v$, where $n v$ denotes the number of nodes. Unless otherwise stated we have used a uniform time step $\delta$. The energy for the discrete curve $u_{h}^{m}$ is calculated as follows

$$
E\left(u_{h}^{m}\right)=\frac{1}{2} \int_{S^{1}} I_{h}\left(\frac{\left|\kappa_{h}^{m}\right|^{2}}{\phi\left(\tau_{h}^{m}\right)}\right)\left|u_{h x}^{m}\right| d x+\lambda \int_{S^{1}} \phi\left(\tau_{h}^{m}\right)\left|u_{h x}^{m}\right| d x .
$$

First of all we show experiments that do not impose any constraint on the length of the curve, thus $\lambda=0$. 
Experiments with no constraint on the length $(\lambda=0)$

We know from the theory that Wulff shapes expand self-similarly in time (recall Example 3.1). Our first sets of experiments study this behavior for different choices of parameters $\sigma$ and $\tilde{\sigma}$ (the parameter $\hat{\sigma}$ plays no role since $\lambda=0$ ). We will measure the following errors

$$
\begin{aligned}
E(\infty, \infty, r) & :=\sup _{1 \leqslant m \leqslant m_{T}} \sup _{0 \leqslant j \leqslant N}\left|\gamma^{*}\left(u_{h}^{m}(j h)\right)-r(m \delta)\right|, \\
E(\infty, 2, r) & :=\sup _{1 \leqslant m \leqslant m_{T}}\left(\int_{S^{1}}\left(\gamma^{*}\left(u_{h}^{m}(\xi)\right)-r(m \delta)\right)^{2} d \xi\right)^{1 / 2},
\end{aligned}
$$

where $r(t)=(1+2 t)^{\frac{1}{4}}$.

\section{TestC1-Unit circle expanding self-similarly in isotropic space}

For the following tests (a), (b) and (c), the initial curve is given by the unit circle $u_{0}(\theta)=$ $(\cos \theta, \sin \theta), \theta \in[0,2 \pi]$, and we work in the isotropic setting, thus $\gamma(\cdot)=\gamma^{*}(\cdot)=|\cdot|$. The final time is $T=1$ and the time step is chosen as $\delta=0.1 h^{2}$.

(a) TestC1-a: here $\sigma=1, \tilde{\sigma}=1$. The results are shown in Table 1 .

TABle 1. TestC1-a: $\lambda=0, \delta=0.1 h^{2}, \sigma=\tilde{\sigma}=1$

\begin{tabular}{lllllll}
\hline$n v$ & $h$ & final $T$ & $E(\infty, \infty, r)$ & eoc & $E(\infty, 2, r)$ & eoc \\
\hline 10 & 0.628318531 & 1.02643887 & 0.0163426516 & - & 0.10334087 & - \\
20 & 0.314159265 & 1.00669966 & 0.00391720658 & 2.0607 & 0.0259083662 & 1.9959 \\
40 & 0.157079633 & 1.00176486 & 0.000969443211 & 2.0145 & 0.00648141865 & 1.999 \\
80 & 0.0785398163 & 1.00053116 & 0.000241754233 & 2.0036 & 0.00162062164 & 1.9997 \\
160 & 0.0392699082 & 1.00006852 & $6.03998004 \mathrm{E}-05$ & 2.0009 & 0.000405160689 & 1.9999 \\
\hline
\end{tabular}

(b) TestC1-b: here $\sigma=1, \tilde{\sigma}=100$. In this test we see how the error increases by choosing a value for $\tilde{\sigma}$ bigger that one (the flow is modified: recall also Remark 6.4). Note however that the convergence rate is basically preserved. The results are shown in Table 2.

TABLE 2. TestC1-b: $\lambda=0, \delta=0.1 h^{2}, \sigma=1, \tilde{\sigma}=100$

\begin{tabular}{lllllll}
\hline$n v$ & $h$ & final $T$ & $E(\infty, \infty, r)$ & eoc & $E(\infty, 2, r)$ & eoc \\
\hline 10 & 0.628318531 & 1.02643887 & 0.152760322 & - & 0.483283335 & - \\
20 & 0.314159265 & 1.00669966 & 0.0507761028 & 1.589 & 0.154442473 & 1.6457 \\
40 & 0.157079633 & 1.00176486 & 0.013501761 & 1.911 & 0.0408327279 & 1.9192 \\
80 & 0.0785398163 & 1.00053116 & 0.00342232288 & 1.98 & 0.0103391907 & 1.9816 \\
160 & 0.0392699082 & 1.00006852 & 0.000858414896 & 1.9952 & 0.0025927508 & 1.9955 \\
\hline
\end{tabular}

(c) TestC1-c: taking $\sigma$ larger than one is not a good idea. If we take $\tilde{\sigma}=1$, and increase $\sigma$, things go wrong, in the sense that for instance the energy ( see (6.14) with $\lambda=0$ ) does not decrease as it 

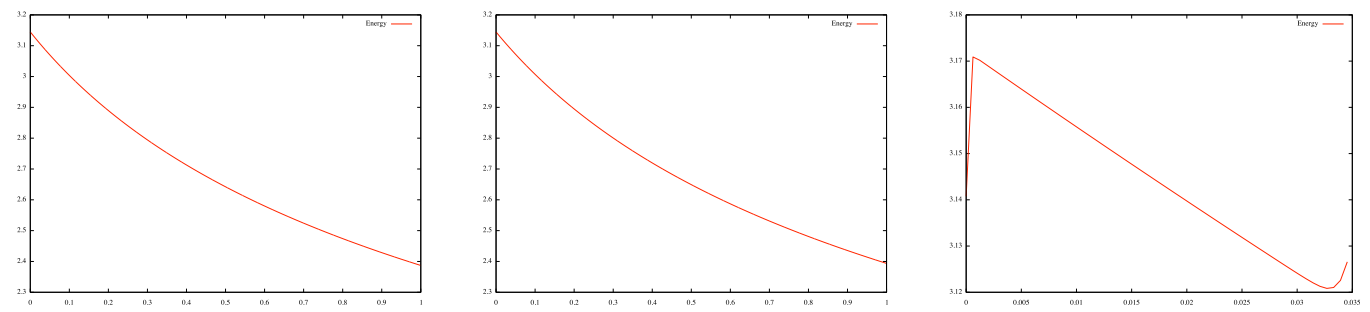

FIG. 1. TestC1-c: isotropic Willmore flow $(\lambda=0)$ : a unit circle is expanding. The energy is depicted for: $\sigma=\tilde{\sigma}=1$ as in test a) (left), $\sigma=1, \tilde{\sigma}=100$ as in test (b) (middle), $\sigma=15, \tilde{\sigma}=1$ (right).

should. In Figure 1 we show the evolution of the energy on the time interval $[0,1]$, when $n v=80$, $\delta=0.1 h^{2}, \lambda=0$ for choices a) $\sigma=1, \tilde{\sigma}=1$, b) $\sigma=1, \tilde{\sigma}=100$ and c) $\sigma=15, \tilde{\sigma}=1$. In view of this behaviour it seems reasonable to keep $\sigma=1$ throughout the following tests.

TestC2: Wulff shape expanding self-similarly in anisotropic space

Here we take the anisotropy to be defined through

$$
\phi(x)=\sqrt{4 x_{1}^{2}+x_{2}^{2}}, \quad\left(x \in \mathbb{R}^{2}\right) .
$$

Hence the corresponding map $\gamma$ is described by the norm $\gamma(x)=\sqrt{x_{1}^{2}+4 x_{2}^{2}}$ and its dual (whose unit ball is the Wulff shape $I$, recall (2.6)) is given by $\gamma^{*}(x)=\sqrt{x_{1}^{2}+\frac{x_{2}^{2}}{4}}$. As initial curve we take the boundary of the Wulff shape, precisely

$$
u_{0}(\theta)=\left(\frac{\cos \theta}{\sqrt{\cos ^{2} \theta+4 \sin ^{2} \theta}}, \frac{4 \sin \theta}{\sqrt{\cos ^{2} \theta+4 \sin ^{2} \theta}}\right), \quad \theta \in[0,2 \pi] .
$$

Here we choose $T=1, \lambda=0, \delta=0.1 h^{3}, \sigma=1$, and $\tilde{\sigma}=100$. The results are shown in Table 3

In Figure 2 we show the evolution for the case $n v=80$.

TABle 3. TestC2: $\lambda=0, \delta=0.1 h^{3}, \sigma=1, \tilde{\sigma}=100$

\begin{tabular}{lllllll}
\hline$n v$ & $h$ & final $T$ & $E(\infty, \infty, r)$ & $e o c$ & $E(\infty, 2, r)$ & $e o c$ \\
\hline 10 & 0.628318531 & 1.01700589 & 0.17854992 & - & 0.507306778 & - \\
20 & 0.314159265 & 1.00150275 & 0.0561985944 & 1.6677 & 0.146557953 & 1.7913 \\
40 & 0.157079633 & 1.00034002 & 0.0120647329 & 2.2197 & 0.0325866208 & 2.1691 \\
80 & 0.0785398163 & 1.00000089 & 0.00201139329 & 2.5845 & 0.00640480912 & 2.347 \\
160 & 0.0392699082 & 1.00000089 & 0.000426727042 & 2.2368 & 0.00133705359 & 2.26 \\
\hline
\end{tabular}




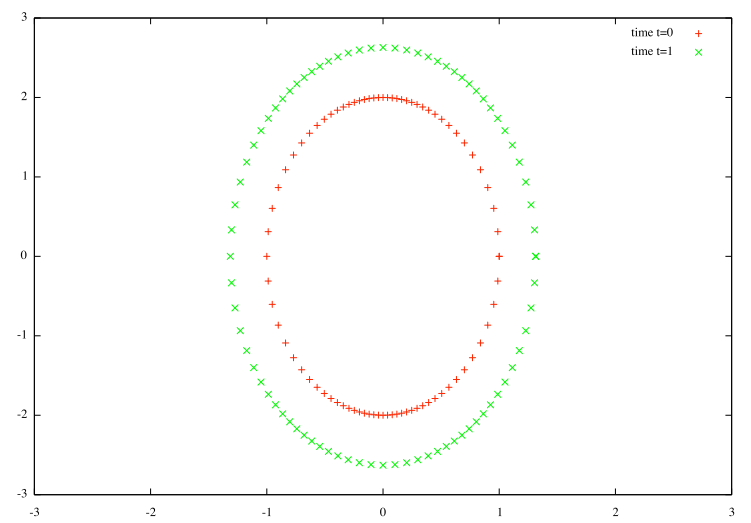

FIG. 2. TestC2: A Wulff shape expanding self similarly

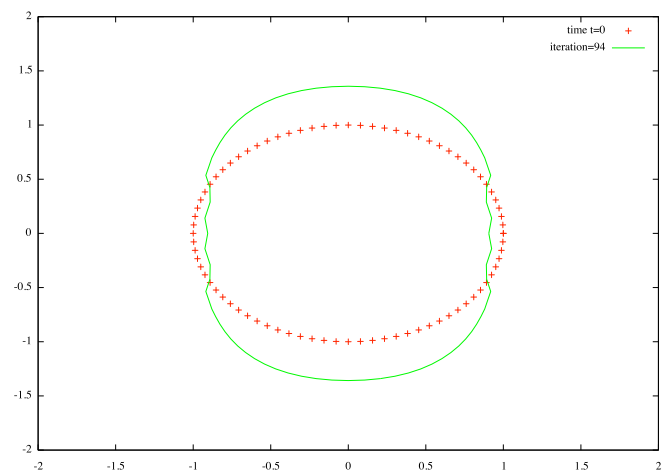

FIG. 3. TestC3: Unstable evolution of a circle expanding in anisotropic setting

\section{TestC3: A unit circle evolving in anisotropic space}

The usefulness and necessity of the stability term introduced by choosing $\tilde{\sigma}>1$ is best demonstrated when a significant change in the curvature takes place during the evolution. For instance we let a unit circle develop under the anisotropy (6.15) without any constraint on the length curve. We take $n v=80, \delta=0.1 h^{2}$, and, as usual, $\lambda=0$ and $\sigma=1$. For choices of $\tilde{\sigma}=1$ and $\tilde{\sigma}=300$ the evolution is not stable: this is manifested through a "corrugation phenomena" as shown in Figure 3, where the evolution for $\tilde{\sigma}=300$ is depicted. On the other hand evolution for $\tilde{\sigma}=1000$ is shown in Figure 4.

\section{TestC4: $L^{p}$-type anisotropy}

Next we consider the anisotropy map

$$
\phi(x)=\left(x_{1}^{4}+x_{2}^{4}\right)^{1 / 4}, \quad\left(x \in \mathbb{R}^{2}\right),
$$




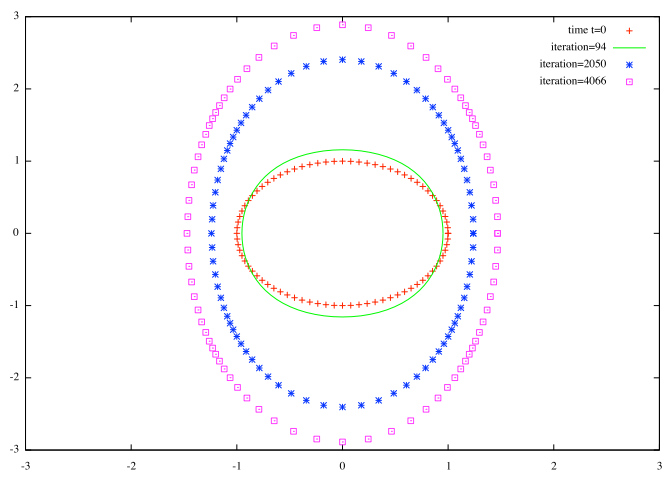

FIG. 4. TestC3: A circle expanding in anisotropic setting

even if it does not satisfy the ellipticity condition (2.5). The Wulff shape in given by $I=\left\{x \in \mathbb{R}^{2}\right.$ : $\left.\gamma^{*}(x) \leqslant 1\right\}$, where

$$
\gamma^{*}(x)=\left(\left|x_{1}\right|^{q}+\left|x_{2}\right|^{q}\right)^{1 / q}, \quad q=\frac{4}{3} .
$$

The initial curve is given by the boundary of the Wulff shape, thus

$$
u_{0}(\theta)=\frac{1}{\left(|\cos \theta|^{4 / 3}+|\sin \theta|^{4 / 3}\right)^{3 / 4}}(\cos \theta, \sin \theta), \quad \theta \in[0,2 \pi]
$$

We choose $\delta=0.1 h^{3}, \lambda=0, \sigma=1, T=0.5$. Evolution at time $t=0$ and $T$ for $n v=160$ and $\tilde{\sigma}=2000$ or $\tilde{\sigma}=4000$ is shown in Figure 5 .

One notices that the grid is improved by taking a larger values of $\tilde{\sigma}$. For $\tilde{\sigma}=8000$ error estimates are collected in Table 4 and a picture of the evolution at time $T=0.5$ (again with $n v=160$ ) is given in Figure 6.
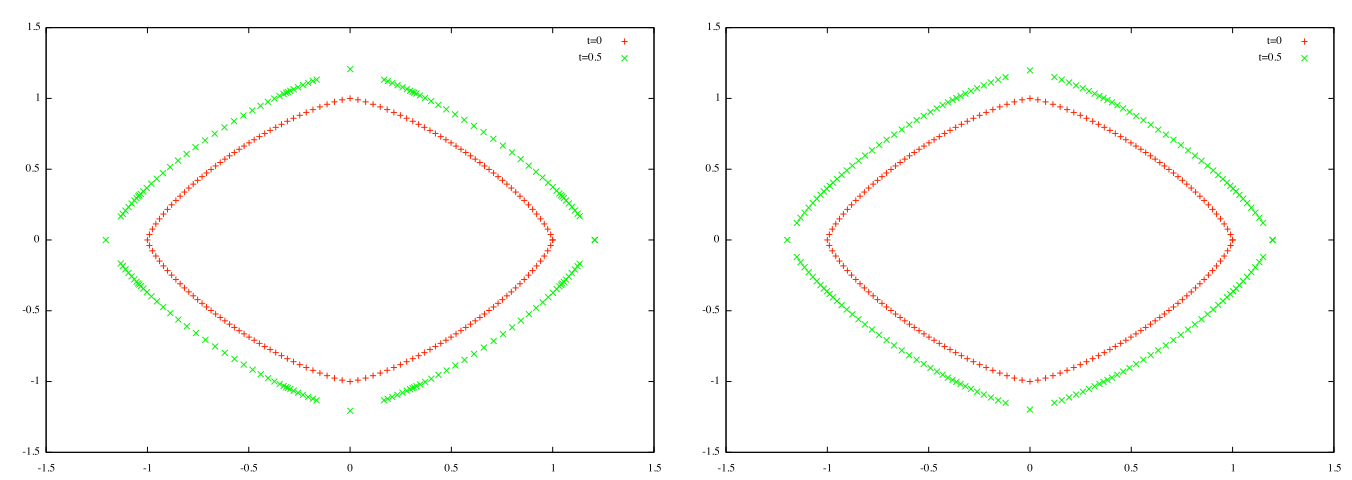

FIG. 5. TestC4: A $L^{q}$-Wulff shape expanding in anisotropic setting. Evolution for $\tilde{\sigma}=2000$ (left) and $\tilde{\sigma}=4000$ (right). 
TABLE 4. TestC4: $\lambda=0, \delta=0.1 h^{3}, \sigma=1, \tilde{\sigma}=8000$

\begin{tabular}{lllllll}
\hline$n v$ & $h$ & final $T$ & $E(\infty, \infty, r)$ & eoc & $E(\infty, 2, r)$ & eoc \\
\hline 10 & 0.628318531 & 0.520905456 & 0.19490107 & - & 0.579459428 & - \\
20 & 0.314159265 & 0.50230169 & 0.179820439 & 0.1161 & 0.458406457 & 0.338 \\
40 & 0.157079633 & 0.500363797 & 0.102260843 & 0.8143 & 0.256153486 & 0.8396 \\
80 & 0.0785398163 & 0.500024666 & 0.0264166087 & 1.9527 & 0.0657562798 & 1.9618 \\
160 & 0.0392699082 & 0.500000443 & 0.00666932488 & 1.9858 & 0.0126488636 & 2.3781 \\
\hline
\end{tabular}

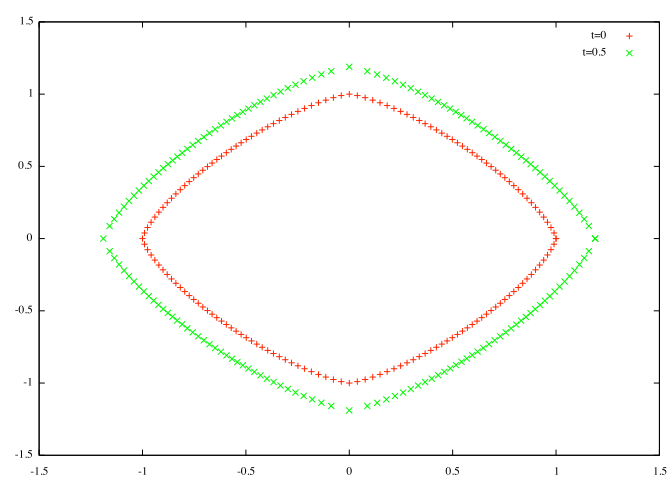

FIG. 6. TestC4: A $L^{q}$-Wulff expanding in anisotropic setting. Evolution for $\widetilde{\sigma}=8000$.

Experiments with a constraint of the length $(\lambda>0)$

TestL1: Evolution of a hypocycloid

Here we briefly compare the initial evolution of a hypocycloid

$$
u_{0}(x)=\left(-\frac{5}{2} \cos (x)+4 \cos (5 x),-\frac{5}{2} \sin (x)+4 \sin (5 x)\right), \quad x \in[0,2 \pi],
$$

under isotropic (cf. [7, Example 4.4]) and anisotropic elastic flow. In both situations we take $n v=$ $200, \sigma=\hat{\sigma}=1, \lambda=0.025$, and variable time step chosen in each iteration as

$$
\delta=0.1 \min _{j}\left|u_{j}-u_{j-1}\right|^{2}
$$

In the isotropic setting $\tilde{\sigma}=1$, whereas for the anisotropic setting we take $\phi$ as in the previous example, see (6.16), and set $\tilde{\sigma}=8000$. Shots for both evolutions are depicted in Figure 7 .

\section{TestL2: Evolution of an asymmetric figure of eight}

Here we work with the initial curve being an asymmetric figure of eight. Its parametrization is given by

$$
u_{0}(x)=\left(\frac{a(\cos x+b) \cos x}{1+(\sin x)^{2}}, \frac{a(\cos x+b) \cos x}{1+(\sin x)^{2}} \sin x\right), \quad x \in[0,2 \pi]
$$



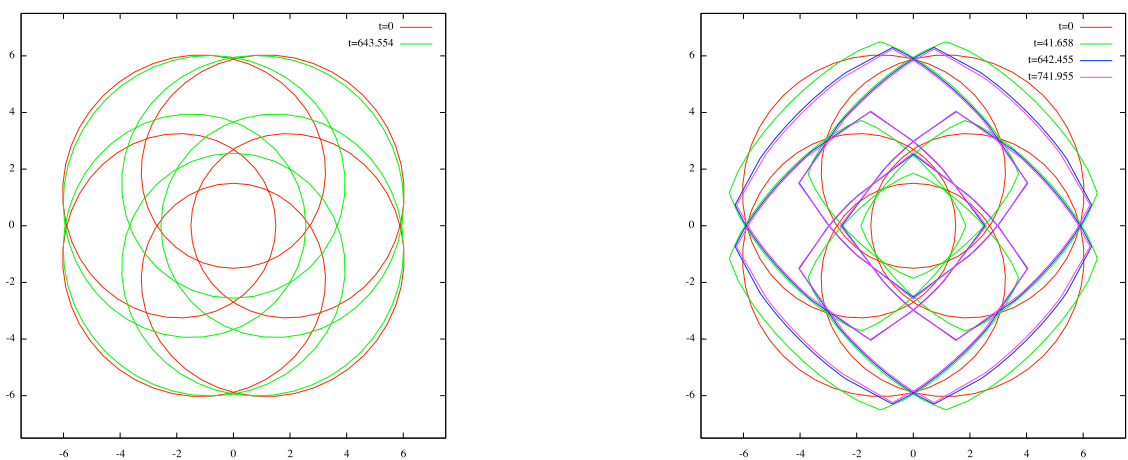

FIG. 7. TestL1: Evolution of a hypocycloid. Isotropic case (left), anisotropic case (right)

where $a=1, b=2$. We take $n v=100, \delta=0.1 h^{3}$, and $\lambda=1$.

(a) TestL2-a First of all we consider the isotropic setting, with $\sigma=\tilde{\sigma}=\hat{\sigma}=1$. The evolution is depicted in Figure 8.
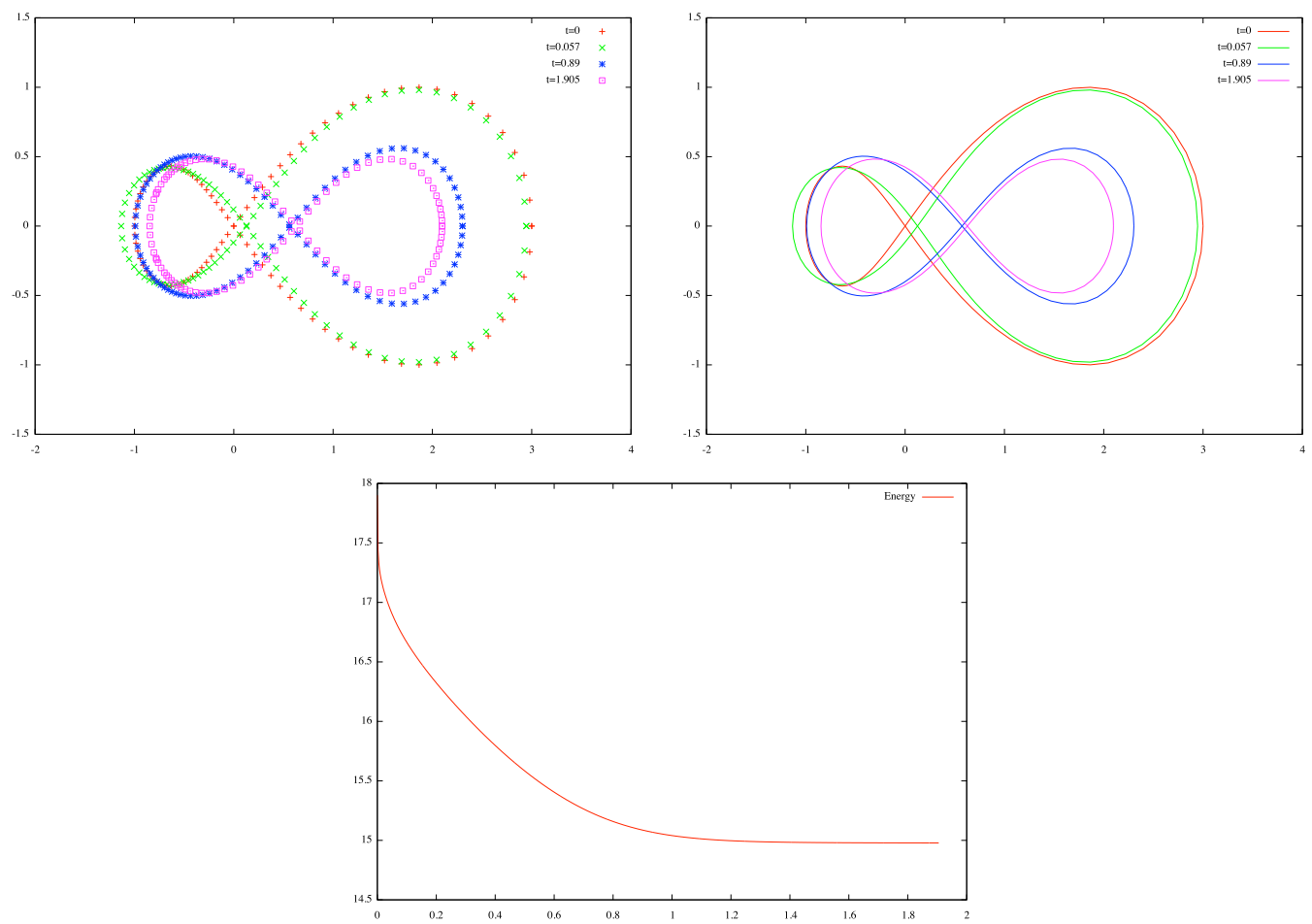

FIG. 8. TestL2-a: Evolution of an asymmetric lemniscate in the isotropic case with $\lambda=1$. Shots of the evolution with and without lines and associated energy evolution 
(b) TestL2-b Here we consider the anisotropy (6.15), with $\hat{\sigma}=\sigma=1$. Several tests have shown that no stability is achieved for $\tilde{\sigma} \in[1,500]$. For $\tilde{\sigma}=1000$ the evolution is depicted in Figure 9. Now the evolutions seems to be stable until there is such a degeneration of the grid that the flows stops behaving as we think it should.

All in all, we recognize that the term associated with $\tilde{\sigma}$ is responsible for the stability of the flow, it generally provides a grid improvement but it does not rule out a grid degeneration. It is now interesting to see if the flows might benefit from a different choice of $\hat{\sigma}$.

(c) TestL2-c Here we consider again the anisotropy (6.15), with $\sigma=1$. Furthermore we take $\tilde{\sigma}=$ 1000 as in the previous test, and set $\hat{\sigma}=500$. Compared to the TestL2-b we notice an improvement of the flow, see Figure 10. However, after the flow has dwelled for some time around the same energy level (see evolution around $t=1$ ), a sudden grid deterioration "destroys" the flow (see evolution around $t=1,3)$.
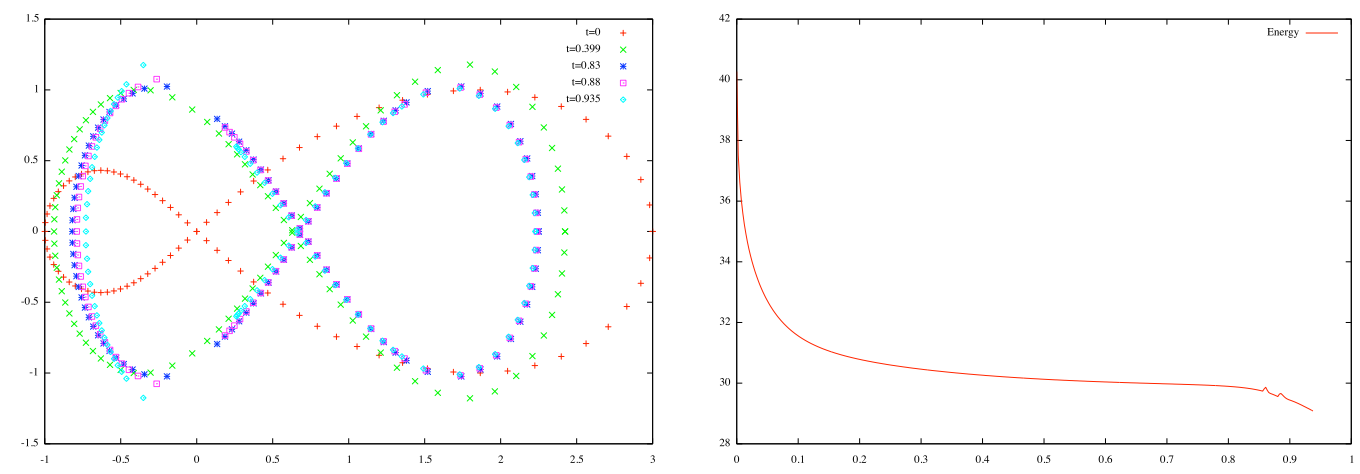

FIG. 9. TestL2-b: Evolution of a asymmetric lemniscate: anisotropic case, $\lambda=1, \tilde{\sigma}=1000$ and associated energy evolution
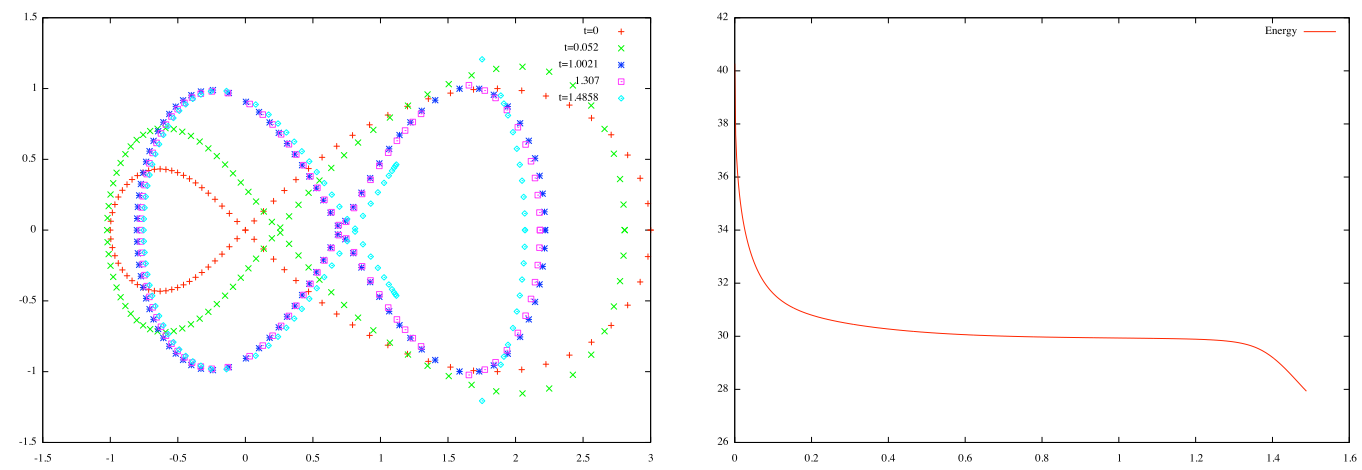

FIG. 10. TestL2-c: Evolution of a asymmetric lemniscate in anisotropic setting with $\lambda=1, \tilde{\sigma}=1000, \hat{\sigma}=500$, and associated energy evolution 
Note that we do not expect stationary points to be attained in finite time: although the author is not aware of long-time existence analysis for the anisotropic Willmore flow, results for the isotropic case in [12] point in this direction.

We conclude this section with a few general remarks. Although stability and a general grid improvement is attained through the introduction of the stability term related to $\tilde{\sigma}$ (and $\hat{\sigma}$ when $\lambda>0$ ), a deterioration of the grid can not be avoided in general. The latter problem is not untypical also for the isotropic setting as mentioned in [7, example 4.3] (it has partly to do with the fact that the discrete flow approximates motion in an entirely normal direction). Therefore, especially for longtime computation, some sort of grid improvement through a redistribution of the nodes would be advisable. This issue is outside the scope of this paper and will be treated elsewhere.

\subsection{The surface case}

Here we consider Problem 4.15 and its semi-discrete formulation Problem 5.1 with $n \geqslant 3$. Arguing similarly to the curve case (cf. (6.10), recall also (4.19) and (4.36)) we can write

$$
\begin{aligned}
\nabla_{\Gamma}^{\gamma} \cdot \varphi & =\nabla_{\Gamma} \cdot \varphi-\gamma_{p_{k}}(v) \frac{v^{l}}{\gamma(v)} \underline{D}_{k} \varphi^{l}=\nabla_{\Gamma} u: \nabla_{\Gamma} \varphi-\gamma_{p_{k}}(v) \frac{v^{l}}{\gamma(v)} \underline{D}_{k} \varphi^{l} \\
& =\sigma \nabla_{\Gamma}(u-I d): \nabla_{\Gamma} \varphi+\nabla_{\Gamma} I d: \nabla_{\Gamma} \varphi-\gamma_{p_{k}}(v) \frac{v^{l}}{\gamma(v)} \underline{D}_{k} \varphi^{l}
\end{aligned}
$$

where $\sigma=1$ and $u=I d$. With the usual notation $f^{m}=f(\cdot, \delta m)$ for time step $\delta>0, m_{T} \delta=T$, and with

$$
S_{h}^{m}=S_{h}(m \delta)=\operatorname{span}\left\{\phi_{1}^{m}, \ldots, \phi_{N}^{m}\right\},
$$

(recall notation of Section 5) we propose following discretization which is the analogue to what we presented in Problem 6.3 for the flow of curves (with $\lambda=0$ ):

Problem 6.5 For a given initial discrete surface $\Gamma_{h}^{0}, u_{h}^{0}$, and time step $\delta$, determine for $m=$ $0,1 \ldots, m_{T}-1$ solutions $\left(u_{h}^{m+1}, \kappa_{h}^{m+1}\right) \in S_{h}^{m} \times S_{h}^{m}$, such that

$$
\begin{aligned}
& \int_{\Gamma_{h}^{m}} \frac{u_{h}^{m+1}-u_{h}^{m}}{\delta \gamma\left(v_{h}^{m}\right)} \cdot \varphi_{h} d V+\frac{\tilde{\sigma}}{2} \int_{\Gamma_{h}^{m}} \frac{\left|\kappa_{h}^{m}\right|^{2}}{\gamma\left(v_{h}^{m}\right)} \nabla_{\Gamma_{h}^{m}}\left(u_{h}^{m+1}-u_{h}^{m}\right): \nabla_{\Gamma_{h}^{m}} \varphi_{h} d V \\
& +\frac{1}{2} \int_{\Gamma_{h}^{m}} \frac{\left|\kappa_{h}^{m}\right|^{2}}{\gamma\left(v_{h}^{m}\right)}\left(\nabla_{\Gamma_{h}^{m}} u_{h}^{m}: \nabla_{\Gamma_{h}^{m}} \varphi_{h}-\gamma_{p_{k}}\left(v_{h}^{m}\right) \frac{\left(v_{h}^{m}\right) l}{\gamma\left(v_{h}^{m}\right)} \underline{D}_{k} \varphi_{h}^{l}\right) d V \\
& -\int_{\Gamma_{h}^{m}} \frac{\left|\kappa_{h}^{m}\right|^{2}}{\gamma\left(v_{h}^{m}\right)} \nabla_{\Gamma_{h}^{m}} u_{h}^{m+1}: \nabla_{\Gamma_{h}^{m}} \varphi_{h} d V-\int_{\Gamma_{h}^{m}} \gamma\left(v_{h}^{m}\right) \nabla_{\Gamma_{h}^{m}}^{\gamma} \cdot \kappa_{h}^{m+1} \nabla_{\Gamma_{h}^{m}}^{\gamma} \cdot \varphi_{h} d V \\
& +\int_{\Gamma_{h}^{m}} \gamma\left(v_{h}^{m}\right) \underline{D}_{l}^{\gamma} \varphi_{h}^{s} \underline{D}_{s}^{\gamma}\left(\kappa_{h}^{m+1}\right)^{l} d V-\int_{\Gamma_{h}^{m}}\left(v_{h}^{m}\right)^{s}\left(v_{h}^{m}\right)^{l} \gamma_{p_{k} p_{j}}\left(v_{h}^{m}\right) \underline{D}_{j}^{\gamma} \varphi_{h}^{s} \underline{D}_{k}^{\gamma}\left(\kappa_{h}^{m+1}\right)^{l} d V=0, \\
& \int_{\Gamma_{h}^{m}} \frac{\kappa_{h}^{m+1}}{\gamma\left(v_{h}^{m}\right)} \cdot \psi_{h} d V+\int_{\Gamma_{h}^{m}} \gamma\left(v_{h}^{m}\right) \nabla_{\Gamma_{h}^{m}} u_{h}^{m+1}: \nabla_{\Gamma_{h}^{m}} \psi_{h}-\gamma_{p_{k}}\left(v_{h}^{m}\right)\left(v_{h}^{m}\right)_{l} \underline{D}_{k} \psi_{h}^{l} d V=0
\end{aligned}
$$

for all $\varphi_{h}, \psi_{h} \in S_{h}^{m}$. Here $u_{h}^{j}(x)=x$ for $x \in \Gamma_{h}^{j}$ for all $j=0, \ldots, m_{T}$. Also $\tilde{\sigma}=1+\tilde{\mu}$ with $\tilde{\mu} \geqslant 0$ to be chosen appropriately as stability term. (Moreover, $\underline{D}_{l} f$ denotes the $l$-th spacial 
component of $\nabla_{\Gamma_{h}^{m}} f$ and $\underline{D}_{l}^{\gamma} f$ the $l$-th spacial component of $\left.\left(\tilde{P}^{\gamma}\right)^{m} \nabla_{\Gamma_{h}^{m}} f\right)$. We set

$$
\Gamma_{h}^{m+1}=u_{h}^{m+1}\left(\Gamma_{h}^{m}\right) .
$$

Notice that in the isotropic case with $\tilde{\sigma}=1$ and $\gamma(p)=|p|$ (from which it follows $\gamma^{\prime \prime}(v)=$ $I-v \otimes v)$ we recover [10, Algorithm 6]. We can express the solution $\left(u_{h}^{m+1}, \kappa_{h}^{m+1}\right)$ as

$$
u_{h}^{m+1}(x)=\sum_{j=1}^{N} u_{j} \phi_{j}^{m}(x), \quad \kappa_{h}^{m+1}(x)=\sum_{j=1}^{N} \kappa_{j} \phi_{j}^{m}(x), \quad x \in \Gamma_{h}^{m},
$$

with coefficients $u_{j}, \kappa_{j} \in \mathbb{R}^{n}$. Using the notation

$$
u^{r}=\left(u_{1}^{r}, \ldots, u_{N}^{r}\right), \quad \kappa^{r}=\left(\kappa_{1}^{r}, \ldots, \kappa_{N}^{r}\right), \quad(r=1, \ldots, n),
$$

as well as the symmetric $(N \times N)$-matrices $M, Q, S$ with entries

$$
\begin{aligned}
& M_{k l}=\int_{\Gamma_{h}^{m}} \frac{1}{\gamma\left(v_{h}^{m}\right)} \phi_{k}^{m} \phi_{l}^{m} d V, \quad Q_{k l}=\frac{1}{2} \int_{\Gamma_{h}^{m}} \frac{\left|\kappa_{h}^{m}\right|^{2}}{\gamma\left(v_{h}^{m}\right)} \nabla_{\Gamma_{h}^{m}} \phi_{k}^{m} \cdot \nabla_{\Gamma_{h}^{m}} \phi_{l}^{m} d V, \\
& S_{k l}=\int_{\Gamma_{h}^{m}} \gamma\left(v_{h}^{m}\right) \nabla_{\Gamma_{h}^{m}} \phi_{k}^{m} \cdot \nabla_{\Gamma_{h}^{m}} \phi_{l}^{m} d V,
\end{aligned}
$$

and $(N \times N)$ - matrices $S^{r s}, R^{r s}(r, s=1, \ldots, n)$ with entries

$$
\begin{aligned}
\left(S^{r s}\right)_{k l} & =\int_{\Gamma_{h}^{m}} \gamma\left(v_{h}^{m}\right) \underline{D}_{r}^{\gamma} \phi_{k}^{m} \underline{D}_{s}^{\gamma} \phi_{l}^{m} d V \\
\left(R^{r s}\right)_{k l} & =\int_{\Gamma_{h}^{m}}\left(v_{h}^{m}\right)_{r}\left(v_{h}^{m}\right)_{s} \sum_{j, q=1}^{n} \gamma_{p_{q} p_{j}}\left(v_{h}^{m}\right) \underline{D}_{j} \phi_{k}^{m} \underline{D}_{q} \phi_{l}^{m} d V
\end{aligned}
$$

and right-hand sides $b^{r}, v^{r} \in \mathbb{R}^{N}(r=1, \ldots, n)$ with

$$
\begin{aligned}
b_{k}^{r}=\frac{1}{\delta} \int_{\Gamma_{h}^{m}} \frac{x_{r}}{\gamma\left(v_{h}^{m}\right)} \phi_{k}^{m} d V+\frac{\tilde{\sigma}-1}{2} \int_{\Gamma_{h}^{m}} \frac{\left|\kappa_{h}^{m}\right|^{2}}{\gamma\left(\nu_{h}^{m}\right)} \nabla_{\Gamma_{h}^{m}} \phi_{k}^{m} \cdot \nabla_{\Gamma_{h}^{m} x_{r}} d V \\
+\frac{1}{2} \int_{\Gamma_{h}^{m}}\left(v_{h}^{m}\right)_{r} \frac{\left|\kappa_{h}^{m}\right|^{2}}{\gamma\left(\nu_{h}^{m}\right)^{2}} \gamma^{\prime}\left(v_{h}^{m}\right) \cdot \nabla_{\Gamma_{h}^{m}} \phi_{k}^{m} d V
\end{aligned}
$$

and

$$
v_{k}^{r}=\int_{\Gamma_{h}^{m}} \gamma^{\prime}\left(v_{h}^{m}\right) \cdot \nabla_{\Gamma_{h}^{m}} \phi_{k}^{m}\left(v_{h}^{m}\right)_{r} d V
$$

for $k=1, \ldots, N$, we see (after plugging in $\varphi=\phi_{k}^{m} e_{r}$ and the expressions for $u^{m+1}$ and $\kappa^{m+1}$ in the system of the above Problem 6.5) that in each time step we have to solve a linear system. Precisely we obtain the following Algorithm. 
Algorithm 6.1 For each time step $m=0,1, \ldots, m_{T}-1$ compute the matrices according to (6.18), (6.19), (6.20), (6.21), as well as the vectors (6.22), (6.23) and solve the linear system

$$
\begin{aligned}
& \frac{1}{\delta} M u^{r}+(\tilde{\sigma}-2) Q u^{r}-\sum_{i=1}^{n} S^{r i} \kappa^{i}+\sum_{i=1}^{n} S^{i r} \kappa^{i}-\sum_{i=1}^{n} R^{r i} \kappa^{i}=b^{r} \\
& M \kappa^{r}+S u^{r}=v^{r}
\end{aligned}
$$

for $r=1, \ldots, n$.

We solve the above linear system by the BICGstab-method.

6.2.1 Numerical simulations for the surface case. Here we show a few simulations in the anisotropic setting for $n=3$. It is important to have in mind the theoretical results mentioned in Remark 2.3 and Theorem 2.4. In the following the energy computed is given by the expression (5.3) at the considered time level. Unless otherwise stated we use an adaptive time step of type $\delta=$ ctime $\cdot h^{4}$, where ctime is a constant that will be specified below and $h$ denotes the grid size of the considered discrete surface (thus the biggest side of the triangles of the triangulation). Note that if one uses a moderately coarse triangulation (useful to reduce computation times) or if some triangles increase considerably in size during the evolution, then it is advisable to use a small constant ctime. A relatively small time step is desirable not only for a good approximation of the velocity, but also because working with a semi-discrete scheme some sort of coupling between time step and grid size is expected. Moreover in view of the considerations made on the stability term (Remark 6.4), a small time step should help "controlling" the modification of the flow induced by the $\tilde{\mu}$-term.

No grid smoothing technique has been used for any of the experiments.

\section{TestS1: The stability term is needed}

In our first experiment we show that in general (similarly to the curve case) the introduction of a $\tilde{\mu}$-stability term with $\tilde{\mu}>0$ is necessary. Here we choose $\gamma(x)=\sqrt{x_{1}^{2}+\frac{x_{2}^{2}}{16}+x_{3}^{2}}$, hence the Wulff shape is given by

$$
I=\left\{x \in \mathbb{R}^{3}: \sqrt{x_{1}^{2}+16 x_{2}^{2}+x_{3}^{2}} \leqslant 1\right\} .
$$

As an initial surface we take the unit sphere. Moreover we set $\tilde{\sigma}=1$. As shown in Figure 11 instability shows up: the energy oscillates and the surface "corrugates".

\section{TestS2: Evolution of a unit sphere in an anisotropic setting}

Here we choose $\gamma(x)=\sqrt{x_{1}^{2}+4 x_{2}^{2}+x_{3}^{2}}$, hence the Wulff shape is given by

$$
I=\left\{x \in \mathbb{R}^{3}: \sqrt{x_{1}^{2}+\frac{x_{2}^{2}}{4}+x_{3}^{2}} \leqslant 1\right\} .
$$

As an initial surface we take the unit sphere. We set $\tilde{\sigma}=1000$ and ctime $=0.0001$. Some shots of the evolution are shown in Figure 12. 

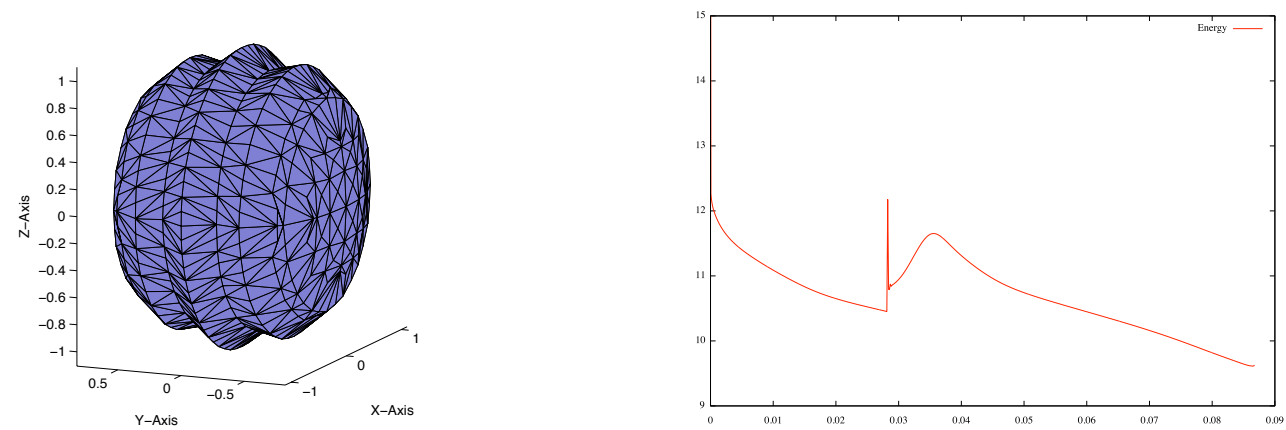

FIG. 11. TestS1: Evolution of a unit sphere in an anisotropic setting with $\tilde{\sigma}=1$ : surface at time $t=0.0865479892$ (left) and oscillating energy (right)
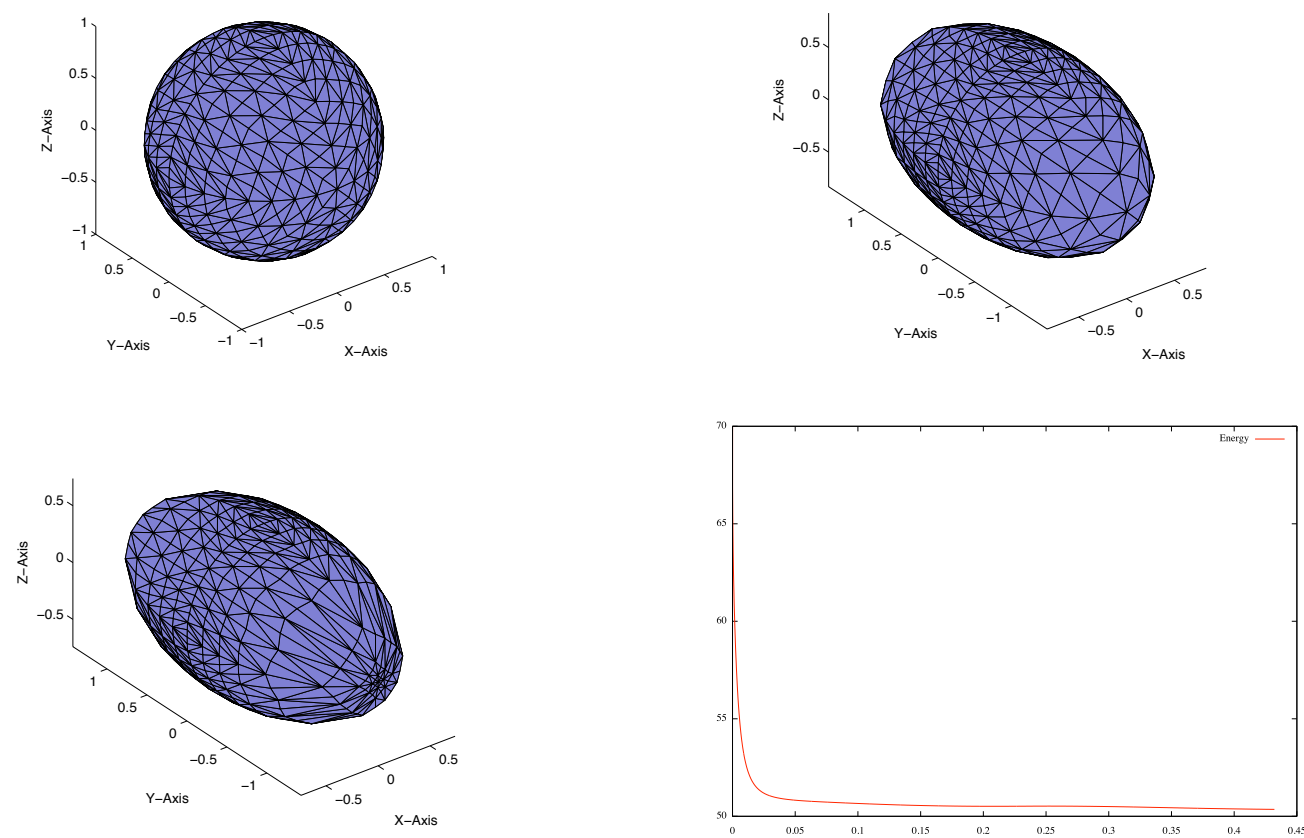

FIG. 12. TestS2: Evolution of a unit sphere in an anisotropic setting with $\tilde{\sigma}=1000$ : initial surface (top left), surface at time $t=0.0409685227$ (top right), surface at time $t=0.432086046$ (bottom left), energy evolution (bottom right)

\section{TestS3: Evolution of a unit sphere and a Clifford torus in an anisotropic setting}

Here we choose $\gamma(x)=\|x\|_{4}=\left(x_{1}^{4}+x_{2}^{4}+x_{3}^{4}\right)^{1 / 4}$, hence the Wulff shape is given by

$$
I=\left\{x \in \mathbb{R}^{3}:\|x\|_{q} \leqslant 1\right\}, \quad \text { with } q=\frac{4}{3} .
$$



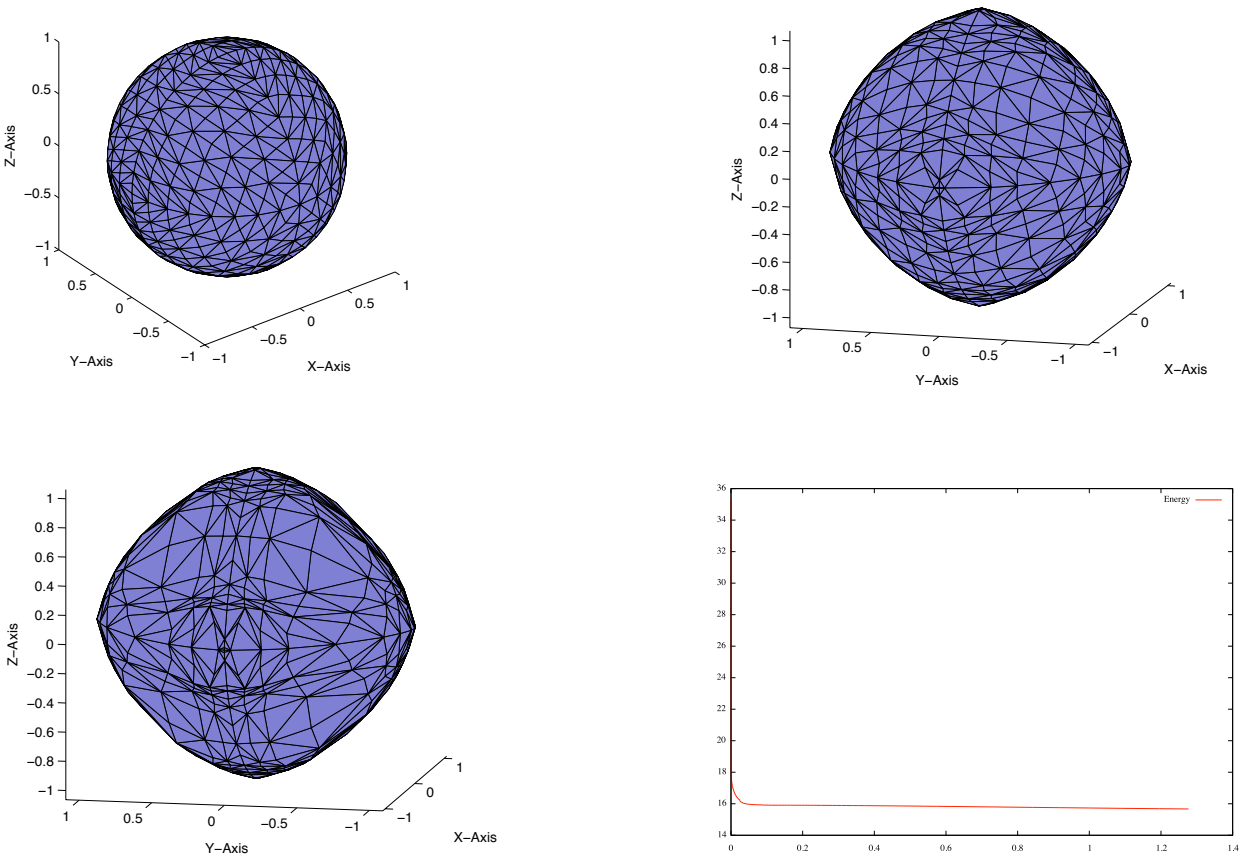

FIG. 13. TestS3-a: Evolution of a unit sphere in anisotropic setting with $\tilde{\sigma}=8000$ : initial surface (top left), surface at time $t=0.18076126$ (top right), surface at time $t=0.700395393$ (bottom left), energy evolution (bottom right)

(a) TestS3-a As in the experiment above we take the initial surface to be a unit sphere. We set $\tilde{\sigma}=8000$ and ctime $=0.0001$. Some shots of the evolution are shown in Figure 13. We notice that the grid degenerates in time.

(b) TestS3-b Here we choose as initial surface a Clifford torus

$$
\Gamma^{0}=\left\{x \in \mathbb{R}^{3}:\left(1-\sqrt{x_{1}^{2}+x_{2}^{2}}\right)^{2}+x_{3}^{2}=\frac{1}{2}\right\} .
$$

Moreover, we set $\tilde{\sigma}=8000$ and ctime $=0.00001$. Some shots of the evolution are shown in Figure 14.

Acknowledgments. I am greatly indebted to G. Dziuk not only for many stimulating conversations, but also for sharing some subroutines and his precious numerical experience for a version of anisotropic Willmore flow. Moreover I would like to thank Jan Steinhilber for several helpful discussions and the DFG Transregional Collaborative Research Centre SFB TR 71 for its generous financial support.

The graphical representations were done with GNUPLOT and Matlab. 

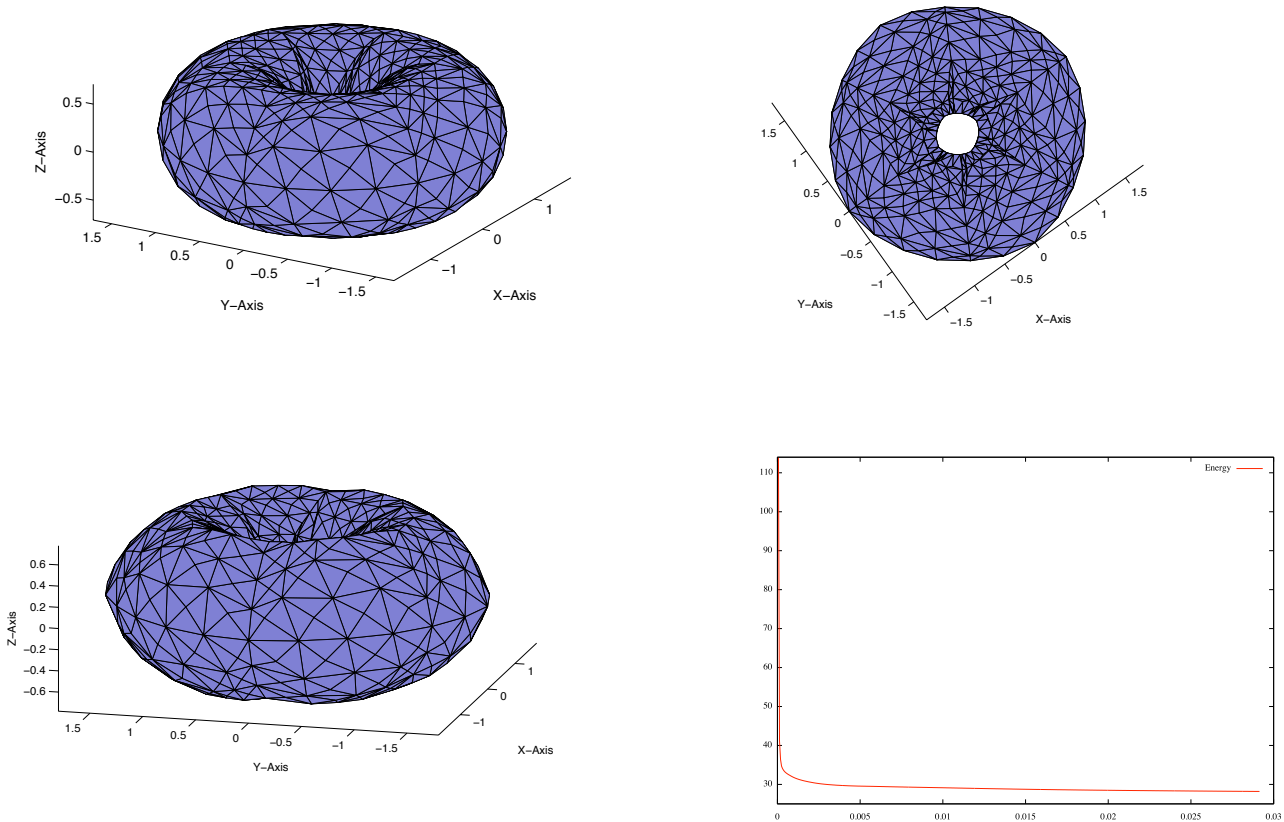

FIG. 14. TestS3-b: Evolution of a Clifford torus in anisotropic setting with $\tilde{\sigma}=8000$ : initial surface (left), surface at time $t=0.0260863107$ viewed from above (top right) and from the side (bottom left), energy evolution (bottom right)

\section{REFERENCES}

1. BARrett, J. W., GARCKe, H., \& NÜRnBERG, R., Parametric approximation of isotropic and anisotropic elastic flow for closed and open curves. Numer. Math. 120 (2012), 489-542. Zb11242.65188 MR2890298

2. Bellettini, G., \& Mugnai, L., Anisotropic geometric functionals and gradient flows. In Nonlocal and abstract parabolic equations and their applications, vol. 86 of Banach Center Publ. Polish Acad. Sci. Inst. Math., Warsaw, 2009, pp. 21-43. Zbl1189.53063 MR2571479

3. Bellettini, G., \& Paolini, M., Anisotropic motion by mean curvature in the context of Finsler geometry. Hokkaido Math. J. 25 (1996), 537-566. Zb10873. 53011 MR1416006

4. Clarenz, U., Enclosure theorems for extremals of elliptic parametric functionals. Calc. Var. Partial Differential Equations 15 (2002), 313-324. Zb11018.53006 MR1938817

5. Clarenz, U., The Wulff shape minimizes an anisotropic Willmore functional. Interfaces Free Bound. 6 3 (2004), 351-359. Zbl1072.35184 MR2095337

6. Clarenz, U., Dziuk, G., \& RUMpF, M., On generalized mean curvature flow in surface processing. In Geometric analysis and nonlinear partial differential equations. Springer, Berlin, 2003, pp. 217-248. Zbl1035.53091 MR2008341

7. Deckelnick, K., \& Dziuk, G., Error analysis for the elastic flow of parametrized curves. Math. Comp. 78 (2009), 645-671. Zbl1198.65183 MR2476555

8. Deckelnick, K., Dziuk, G., \& Elliott, C. M., Computation of geometric partial differential equations and mean curvature flow. Acta Numer. 14 (2005), 139-232. Zbl1113.65097 MR2168343 
9. DiEwald, U., Anisotrope Krümmungsflüsse parametrischer Fläche sowie deren Anwendung in der Flächenverarbeitung. PhD thesis, Univ. of Duisburg-Essen (2005).

10. DzIUK, G., Computational parametric Willmore flow. Numer. Math. 111 (2008), 55-80. Zb11158.65073 MR2448203

11. Dziuk, G., \& Elliott, C. M., Finite elements on evolving surfaces. IMA J. Numer. Anal. 272 (2007), 262-292. Zbl1120.65102 MR2317005

12. Dziuk, G., Kuwert, E., \& Schätzle, R., Evolution of elastic curves in $\mathbb{R}^{n}$ : Existence and computation. SIAM J. Math. Anal. 33 (2002), 1228-1245 (electronic). Zbl1031. 53092 MR1897710

13. Magnus, J. R., \& NeUdecker, H., Matrix differential calculus with applications in statistics and econometrics. Wiley Series in Probability and Mathematical Statistics: Applied Probability and Statistics. John Wiley \& Sons, Ltd., Chichester, 1988. Zb10651. 15001 MR0940471

14. PAlmer, B., Equilibria for anisotropic bending energies. J. Math. Phys. 50 (2009), 023512, 11. Zbl1202 . 49055 MR2494852

15. Perl, R., Pozzi, P., \& RumpF, M., A nested variational time discretization for parametric anisotropic willmore flow. In Singular Phenomena and Scaling in Mathematical Models, M. Griebel, Ed. Springer, 2014. MR3205043

16. PozZI, P. Anisotropic curve shortening flow in higher codimension. Math. Methods Appl. Sci. 3011 (2007), 1243-1281. Zbl1122.65081 MR2334978

17. PozzI, P., Anisotropic mean curvature flow for two-dimensional surfaces in higher codimension: a numerical scheme. Interfaces Free Bound. 104 (2008), 539-576. Zbl1158. 65076 MR2465273

18. PozzI, P., On the gradient flow for the anisotropic area functional. Math. Nachr. 285 (2012), 707-726. Zbl1242.53084 MR2902841

19. Thompson, A. C., Minkowski geometry, vol. 63 of Encyclopedia of Mathematics and its Applications. Cambridge University Press, Cambridge, 1996. Zb10868.52001 MR1406315

20. Willmore, T. J., Riemannian geometry. Oxford Science Publications. The Clarendon Press Oxford University Press, New York, 1993. Zb10797.53002 MR1261641

21. ZeIDLER, E., Nonlinear functional analysis and its applications. III. Springer-Verlag, New York, 1985. Variational methods and optimization, Translated from the German by Leo F. Boron. Zb10583.47051 MR0768749 